This is the final peer-reviewed accepted manuscript of:

Franchi, B., Serapioni, R.P. Intrinsic Lipschitz Graphs Within Carnot Groups. J Geom Anal 26, 1946-1994 (2016).

The final published version is available online at: https://doi.org/10.1007/s12220015-9615-5

Rights / License:

The terms and conditions for the reuse of this version of the manuscript are specified in the publishing policy. For all terms of use and more information see the publisher's website.

This item was downloaded from IRIS Università di Bologna (https://cris.unibo.it/)

When citing, please refer to the published version. 


\title{
INTRINSIC LIPSCHITZ GRAPHS WITHIN CARNOT GROUPS
}

\author{
BRUNO FRANCHI AND RAUL PAOLO SERAPIONI
}

\begin{abstract}
A Carnot group is a connected, simply connected, nilpotent Lie group with stratified Lie algebra. We study the notions of intrinsic graphs and of intrinsic Lipschitz graphs within Carnot groups. Intrinsic Lipschitz graphs are the natural local analogue inside Carnot groups of Lipschitz submanifolds in Euclidean spaces, where 'natural' emphasizes that the notion depends only on the structure of the algebra. Intrinsic Lipschitz graphs unify different alternative approaches through Lipschitz parameterizations or level sets. We provide both geometric and analytic characterizations and a clarifying relation between these graphs and Rumin's complex of differential forms.
\end{abstract}

\section{INTRODUCTION}

The aim of this paper is to provide a comprehensive presentation of the notion of intrinsic graph and, more specifically, of intrinsic Lipschitz graph within Carnot groups (connected, simply connected, nilpotent Lie groups). In particular, we prove that there is a biunivocal correspondence between intrinsic graphs and covectors of the so-called Rumin's complex of differential forms in Carnot groups. The notion of intrinsic Lipschitz graph appeared for the first time in [24], [5], [26] [20], in connection with the problem of finding a good invariant notion of regular submanifold in Carnot groups. Since this problem lies at the very roots of the notion of intrinsic graph, we shall discuss it extensively later on in this Introduction. Finally, in the last section of this paper, we prove an extension theorem for intrinsic Lipschitz functions, as well as the Ahlfors regularity of 1-codimensional intrinsic graphs.

It is well known that the Lie algebra $\mathfrak{g}$ of the left-invariant vector fields of a Carnot group $\mathbb{G}$ has finite dimension $n$, and admits a step $\kappa$ stratification, i.e. there exist linear subspaces (so-called layers) $\mathfrak{g}_{1}, \ldots, \mathfrak{g}_{\kappa}$ such that

$$
\mathfrak{g}=\mathfrak{g}_{1} \oplus \cdots \oplus \mathfrak{g}_{\kappa}, \quad\left[\mathfrak{g}_{1}, \mathfrak{g}_{i}\right]=\mathfrak{g}_{i+1}, \quad \mathfrak{g}_{\kappa} \neq\{0\},
$$

where $\mathfrak{g}_{i}=\{0\}$ if $i>\kappa$, and $\left[\mathfrak{g}_{1}, \mathfrak{g}_{i}\right]$ is the subspace of $\mathfrak{g}$ generated by the commutators $[X, Y]$ with $X \in \mathfrak{g}_{1}$ and $Y \in \mathfrak{g}_{i}$. The Lie algebra $\mathfrak{g}$ can be endowed with a scalar product that makes the decomposition (1) orthogonal. We refer to the first layer $\mathfrak{g}_{1}$ as to the horizontal layer. It plays a key role in our theory, since it generates all of $\mathfrak{g}$ by commutations.

Through exponential coordinates, the group $\mathbb{G}$ can be identified with $\left(\mathbb{R}^{n}, \cdot\right)$, the Euclidean space $\mathbb{R}^{n}$ endowed with a (generally non-commutative) group law.

Carnot groups are endowed with two families of transformations: the (left) translations $\tau_{p}: \mathbb{G} \rightarrow$ $\mathbb{G}$ defined as $q \mapsto \tau_{p} q:=p \cdot q$, and the non-isotropic group dilations $\delta_{\lambda}: \mathbb{G} \rightarrow \mathbb{G}$.

Euclidean spaces are commutative Carnot groups, and, more precisely, the only commutative Carnot groups. The simplest but, at the same time, non-trivial instance of non-Abelian Carnot groups is provided by Heisenberg groups $\mathbb{H}^{n}$ Carnot groups are endowed with an intrinsic geometry, the so-called Carnot-Carathéodory geometry (see for instance, choosing in a wide literature, [9], [28] [23]). From now on, the adjective "intrinsic" is meant to emphasize a privileged role played

Date: May 3, 2015.

B.F. is supported by MURST, Italy, by University of Bologna, funds for selected research topics, by GNAMPA of INdAM, Italy and by MAnET Marie Curie Initial Training Network.

R.P.S. is supported by MURST, Italy, Università of Trento, Italy and by MAnET Marie Curie Initial Training Network. 
by the horizontal layer and by group translations and dilations. In other words, "intrinsic" notions or properties in the group $\mathbb{G}$ are those depending only on the structure of its Lie algebra $\mathfrak{g}$. In particular, an intrinsic notion must be invariant under group translations and group dilations.

It is also well known that non commutative Carnot groups, endowed with their Carnot-Carathéodory distance (briefly, cc-distance), are not Riemannian manifolds because their Carnot-Carathédory distance makes them metric spaces not Riemannian at any scale ([40]). In fact, they are particular instances of the so-called sub-Riemannian metric spaces.

In the last few years, there has been a general attempt aimed to carry on geometric analysis in non-Euclidean structures, and, in particular, to develop a notion of rectifiable set in metric spaces, and, specifically, in sub-Riemannian metric structures and in Carnot groups. For different notions of rectifiability we refer the reader to [2], [3], [21], [25], [37], [33] and to the references therein. It is worth noticing that, besides their own geometric interest, rectifiable sets in Lie groups appear in several applications, such as theoretical computer science, geometry of Banach spaces, mathematical models in neurosciences (see e.g. [12], [11]).

In Euclidean spaces, rectifiable sets are obtained, up to a negligible subset, by "gluing up" countable families of $C^{1}$ or of Lipschitz submanifolds. Hence, understanding the objects that, within Carnot groups, naturally take the role of $C^{1}$ or of Lipschitz submanifolds is preliminary in order to develop a satisfactory theory of intrinsic rectifiable sets. It has been clear for a long time that considering Euclidean regular submanifolds, even in Heisenberg groups, may be both too general and too restrictive (see e.g. [31] for a striking example related to the second instance). More intrinsic definitions are necessary.

In the Euclidean setting $C^{1}$ submanifolds can be locally viewed, equivalently, as (i) $C^{1}$ injective images of a fixed "parameter space" (usually an open subset of a linear space); (ii) non-critical level sets of $C^{1}$-functions; (iii) graphs of $C^{1}$ maps between complementary linear subspaces.

Notion (i) was the first one to be extended to the setting of general metric spaces (see e.g. [17], [2] where the parameter spaces are open subsets of Euclidean spaces). When working with a Carnot group $\mathbb{G}$, it is natural to think of using more general spaces of parameters, i.e. open subsets of homogeneous subgroups of $\mathbb{G}$ (see [37] and [33]). A very special instance are horizontal curves, usually defined as images of Lipschitz maps $\mathbb{R} \rightarrow \mathbb{G}$.

Also notion (ii) has been largely studied in the recent literature, starting from the implicit function theorem in Carnot groups proved in [21] and [22].

Nevertheless, both approach (i) and (ii) have intrinsic limitations appearing already inside Heisenberg groups. Indeed, differently from $\mathbb{R}^{n}$ - where embedded submanifolds are equivalently defined as non-critical level sets or as images of injective differentiable maps - in $\mathbb{H}^{n}$, low dimensional regular surfaces cannot be seen as non critical level sets and low codimensional ones cannot be seen as (bilipschitz) images of open sets of $\mathbb{R}^{n}$. The reasons are rooted in the algebraic structure of $\mathbb{H}^{n}$; indeed, low dimensional horizontal subgroups of $\mathbb{H}^{n}$ are not normal subgroups, hence they cannot appear as kernels of homogeneous homomorphisms $\mathbb{H}^{n} \rightarrow \mathbb{R}^{n-d}$; on the other side, injective homogeneous homomorphism $\mathbb{R}^{d} \rightarrow \mathbb{H}^{n}$ do not exist, if $d \geq n+1$ (see [2] and [32]). One could object that this difficulty might be overcome using subgroups of $\mathbb{G}$ instead of $\mathbb{R}^{d}$ as a parameter space. Indeed this is not the case as examples in [8] and in [4] show.

On the other hand, the notion of graphs within Carnot groups is definitely more delicate, since Carnot groups in general are not cartesian products of subgroups (unlike Euclidean spaces). A notion of intrinsic graph fitting the structure of the group $\mathbb{G}$ is needed.

Intrinsic graphs appeared naturally in [24], [5], [26] in relation with non critical level sets of differentiable functions from $\mathbb{G}$ to $\mathbb{R}^{k}$. Indeed, implicit function theorems for groups ([21], [25], [22], [13], [14]) can be rephrased stating precisely that these level sets are always, locally, intrinsic graphs.

An intrinsic graph inside $\mathbb{G}$ is associated with a decomposition of the ambient group $\mathbb{G}$ as a product $\mathbb{G}=\mathbb{M} \cdot \mathbb{H}$ of two homogeneous complementary subgroups $\mathbb{M}, \mathbb{H}$ (a Lie subgroup of $\mathbb{G}$ is 
said to be an homogeneous subgroup if it is invariant under group dilations. See Definition 5) and the idea of intrinsic graph is the following one: let $\mathbb{M}, \mathbb{H}$ be complementary homogeneous subgroups of a group $\mathbb{G}$, then the intrinsic (left) graph of $f: \mathcal{A} \subset \mathbb{M} \rightarrow \mathbb{H}$ is the set

$$
\operatorname{graph}(f)=\{g \cdot f(g): g \in \mathcal{A}\} \text {. }
$$

Describing regular submanifolds as (intrinsic differentiable) graphs is more general and flexible than using parametrizations or level sets. For instance, already in Heisenberg groups, both non critical level sets and images of regular maps are locally intrinsic differentiable graphs (see [5]). The same happens for one codimensional submanifolds in general Carnot groups.

Intrinsic graphs are 'intrinsic' since they keep being intrinsic graphs after left translations or homogeneous automorphisms of the group (dilations in particular): see Proposition 2.2.18. We stress that, in general, Euclidean graphs may fail to be intrinsic graphs. At the same time, in general, intrinsic graphs may fail to be Euclidean graphs.

Hence the very existence of intrinsic graphs depends on the possibility of splitting $\mathbb{G}$ as a product of complementary subgroups hence it depends on the structure of the algebra $\mathfrak{g}$.

There is an interesting relationship between all possible decompositions of a Carnot group $\mathbb{G}$ as a product of complementary subgroups and the structure of the Rumin's complex $\left(E_{0}^{*}, d_{c}\right)$ of differential forms in $\mathbb{G}$. Rumin's theory would need a quite long technical introduction, and hence we refer to the original Rumin's papers [38] and [39], as well as to [10] and [27] for an exhaustive presentation. In this paper we prove the existence of a canonical explicit biunivocal correspondence between (equivalence classes of parallel) simple $h$-covectors in $E_{0}^{h}$ and group decompositions, akin to the correspondence in Euclidean spaces between linear manifolds and equivalence classes of covectors (see Theorem 2.2.7).

What are the right intrinsic notions of Lipschitz function or differentiable function when dealing with functions acting between complementary subgroups? In the second part of this paper we study mainly intrinsic Lipschitz functions.

If the group $\mathbb{G}$ admits a decomposition $\mathbb{G}=\mathbb{M} \cdot \mathbb{H}$, then two canonical projections $\mathbf{P}_{\mathbb{M}}$ and $\mathbf{P}_{\mathbb{H}}$ are naturally defined by the identity $\mathbf{P}_{\mathbb{M}} g \cdot \mathbf{P}_{\mathbb{H}} g \equiv g$ for $g \in \mathbb{G}$. Even if $\mathbf{P}_{\mathbb{M}}$ and $\mathbf{P}_{\mathbb{H}}$ are not in general Lipschitz maps with respect to the cc-distance in $\mathbb{G}$ they yield a notion of intrinsic cone: if $g \in \mathbb{G}$ and $\beta \geq 0$ the cones $C_{\mathbb{M}, \mathbb{H}}(g, \beta)$, with basis $\mathbb{M}$, axis $\mathbb{H}$, vertex $g$, opening $\beta$ are defined as follows:

$$
\text { if } g=e \text {, then } \quad C_{\mathbb{M}, \mathbb{H}}(e, \beta):=\left\{p:\left\|\mathbf{P}_{\mathbb{M}} p\right\| \leq \beta\left\|\mathbf{P}_{\mathbb{H}} p\right\|\right\} \text {, }
$$

and, in general,

$$
C_{\mathbb{M}, \mathbb{H}}(g, \beta):=g \cdot C_{\mathbb{M}, \mathbb{H}}(e, \beta) .
$$

Thus we can say that $f: \mathcal{E} \subset \mathbb{M} \rightarrow \mathbb{H}$ is intrinsic L-Lipschitz in $\mathcal{E}$ if there is $L>0$ such that

$$
C_{\mathbb{M}, \mathbb{H}}(p, 1 / L) \cap \operatorname{graph}(f)=\{p\}, \quad \text { for all } p \in \operatorname{graph}(f) \text {. }
$$

By construction, this notion is invariant under group translations. On the other side intrinsic Lipschitz functions are not metric Lipschitz functions with respect to the cc-distance of $\mathbb{G}$ or other natural metrics in the domain and in the target (see Remark 3.1.6). In particular, our theory of intrinsic Lipschitz maps within Carnot groups does not fit in Pansu's theory of Lipschitz maps between Carnot groups (see [36]). Nevertheless intrinsic Lipschitz functions seem to be the right objects to consider inside groups. Among the reasons for this, there is its invariance under left translations (see Theorem 3.1.2) and properties such as the Ahlfors regularity of their graphs (see Theorem 3.2.1). In addition, we recall that a real-valued Lipschitz function defined on any subset of a given metric space $X$ can be extended to a real-valued Lipschitz function defined on all of $X$. In Section 4 we address and solve the analogous extension problem for a "real-valued intrinsic Lipschitz function", i.e. an intrinsic Lipschitz function $f$ acting between subsets of complementary subgroups $\mathbb{M}$ and $\mathbb{H}$, with $\mathbb{H}$ being 1-dimensional and horizontal (and hence isomorphic to $\mathbb{R}$ ). 
Further intrinsic differentiability properties of intrinsic Lipschitz functions are studied in the forthcoming paper [19].

It is a pleasure here to thank Pierre Pansu and Francesco Serra Cassano for several stimulating conversations and precious advices, and the Referee for his careful reading and his helpful suggestions.

The plan of the work is the following:

Section 2 contains basic notions about Carnot groups and in particular the relation between the structure of complementary subgroups of $\mathbb{G}$ and Rumin's complex. Then we study projection operators associated with a group decomposition.

Section 3 is dedicated to a systematic study of intrinsic Lipschitz graphs. Ahlfors regularity of the graph is proved here.

Section 4 is specialized to intrinsic Lipschitz functions with values in one dimensional subgroups. The main result here is an extension theorem for one dimensional intrinsic Lipschitz functions.

\section{NOTATIONS AND DEFINITIONS}

2.1. Carnot groups. For a general account, see e.g. [9, 18, 28]. A graded group of step $\kappa$ is a connected, simply connected Lie group $\mathbb{G}$ whose finite dimensional Lie algebra $\mathfrak{g}$ is the direct sum of $k$ subspaces $\mathfrak{g}_{i}, \mathfrak{g}=\mathfrak{g}_{1} \oplus \cdots \oplus \mathfrak{g}_{\kappa}$, such that

$$
\left[\mathfrak{g}_{i}, \mathfrak{g}_{j}\right] \subset \mathfrak{g}_{i+j}, \quad \text { for } 1 \leq i, j \leq \kappa,
$$

where $\mathfrak{g}_{i}=0$ for $i>\kappa$. We denote as $n$ the dimension of $\mathfrak{g}$ and as $n_{j}$ the dimension of $\mathfrak{g}_{j}$, for $1 \leq j \leq \kappa$.

A Carnot group $\mathbb{G}$ of step $\kappa$ is a graded group of step $\kappa$, where $\mathfrak{g}_{1}$ generates all of $\mathfrak{g}$. That is $\left[\mathfrak{g}_{1}, \mathfrak{g}_{i}\right]=\mathfrak{g}_{i+1}$, for $i=1, \ldots, \kappa$.

Let $X_{1}, \ldots, X_{n}$ be a base for $\mathfrak{g}$ such that $X_{1}, \ldots, X_{m_{1}}$ is a base for $\mathfrak{g}_{1}$ and, for $1<j \leq \kappa$, $X_{m_{j-1}+1}, \ldots, X_{m_{j}}$ is a base for $\mathfrak{g}_{j}$. Here we have $m_{0}=0$ and $m_{j}-m_{j-1}=n_{j}$, for $1 \leq j \leq \kappa$.

Because the exponential map is a one to one diffeomorphism from $\mathfrak{g}$ to $\mathbb{G}$, any $p \in \mathbb{G}$ can be written, in a unique way, as $p=\exp \left(p_{1} X_{1}+\cdots+p_{n} X_{n}\right)$ and we identify $p$ with the n-tuple $\left(p_{1}, \ldots, p_{n}\right) \in \mathbb{R}^{n}$ and $\mathbb{G}$ with $\left(\mathbb{R}^{n}, \cdot\right)$, i.e. $\mathbb{R}^{n}$ endowed with the product $\cdot$. The identity of $\mathbb{G}$ is denoted as $e=(0, \ldots, 0)$.

If $\mathbb{G}$ is a graded group, for all $\lambda>0$, the (non isotropic) dilations $\delta_{\lambda}: \mathbb{G} \rightarrow \mathbb{G}$ are automorphisms of $\mathbb{G}$ defined as

$$
\delta_{\lambda}\left(p_{1}, \ldots, p_{n}\right)=\left(\lambda^{\alpha_{1}} p_{1}, \lambda^{\alpha_{2}} p_{2}, \ldots, \lambda^{\alpha_{n}} p_{n}\right),
$$

where $\alpha_{i}=j$, if $m_{j-1}<i \leq m_{j}$.

We denote the product of $p$ and $q \in \mathbb{G}$ as $p \cdot q$ or more frequently as $p q$. The explicit expression of the group operation · is determined by the Campbell-Hausdorff formula. It has the form

$$
p \cdot q=p+q+\mathcal{Q}(p, q), \quad \text { for all } p, q \in \mathbb{R}^{n},
$$

where $\mathcal{Q}=\left(\mathcal{Q}_{1}, \ldots, \mathcal{Q}_{n}\right): \mathbb{R}^{n} \times \mathbb{R}^{n} \rightarrow \mathbb{R}^{n}$ and each $\mathcal{Q}_{i}$ is a homogeneous polynomial of degree $\alpha_{i}$ with respect to the intrinsic dilations of $\mathbb{G}$. That is

$$
\mathcal{Q}_{i}\left(\delta_{\lambda} p, \delta_{\lambda} q\right)=\lambda^{\alpha_{i}} \mathcal{Q}_{i}(p, q), \quad \text { for all } p, q \in \mathbb{G} \text { and } \lambda>0 .
$$

We collect now further properties of $\mathcal{Q}$ following from Campbell-Hausdorff formula. First of all $\mathcal{Q}$ is antisimmetric, that is

$$
\mathcal{Q}_{i}(p, q)=-\mathcal{Q}_{i}(-q,-p), \quad \text { for all } p, q \in \mathbb{G} .
$$

Each $\mathcal{Q}_{i}(p, q)$ depends indeed only on a section of the components of $p$ and $q$. Precisely

$$
\begin{aligned}
& \mathcal{Q}_{1}(p, q)=\cdots=\mathcal{Q}_{m_{1}}(p, q)=0 \\
& \mathcal{Q}_{j}(p, q)=\mathcal{Q}_{j}\left(p_{1}, \ldots, p_{m_{i-1}}, q_{1}, \ldots, q_{m_{i-1}}\right),
\end{aligned}
$$


if $m_{i-1}<j \leq m_{i}$ and $2 \leq i$. By Proposition 2.2.22 (4) in [9],

$$
\mathcal{Q}_{i}(p, q)=\sum_{k, h} \mathcal{R}_{k, h}^{i}(p, q)\left(p_{k} q_{h}-p_{h} q_{k}\right), \quad \text { for } m_{1}<i \leq n,
$$

where $\mathcal{R}_{k, h}^{i}$ are polynomials, homogenous of degree $\alpha_{i}-\alpha_{k}-\alpha_{h}$ with respect to group dilations, and the sum is extended to all $h, k$ such that $\alpha_{h}+\alpha_{k} \leq \alpha_{i}$. From (5) it follows that

$$
\mathcal{Q}_{i}(p, 0)=\mathcal{Q}_{i}(0, q)=0 \quad \text { and } \quad \mathcal{Q}_{i}(p, p)=\mathcal{Q}_{i}(p,-p)=0 .
$$

Finally, it is useful to think $\mathbb{G}=\mathbb{G}^{1} \oplus \mathbb{G}^{2} \oplus \cdots \oplus \mathbb{G}^{\kappa}$, where $\mathbb{G}^{i}=\exp \left(\mathfrak{g}_{i}\right)=\mathbb{R}^{n_{i}}$ is the $i^{\text {th }}$ layer of $\mathbb{G}$ and to write $p \in \mathbb{G}$ as $\left(p^{1}, \ldots, p^{\kappa}\right)$, with $p^{i} \in \mathbb{G}^{i}$. According to this

$$
p \cdot q=\left(p^{1}+q^{1}, p^{2}+q^{2}+\mathcal{Q}^{2}(p, q), \ldots, p^{\kappa}+q^{\kappa}+\mathcal{Q}^{\kappa}(p, q)\right), \quad \text { for all } p, q \in \mathbb{G} .
$$

We stress that the $\mathcal{Q}^{i}$ 's are vector-valued polynomials for $i=1, \ldots, \kappa$, whereas the $\mathcal{Q}_{j}$ 's are scalar polynomials for $j=1, \ldots, n$.

Remark 2.1.1. Later on it will be useful an extension of (6) to the case of $d>2$ factors.

Let $p, q, r, \ldots$ be $d$ elements of $\mathbb{G}$. Observe that $(p \cdot q \cdot r \cdot \ldots)_{\ell}$ is, by definition, homogeneous of degree $\alpha_{\ell}$ and, by iteration of formula (3), is a polynomial in the components of $p, q, r, \ldots$ that being of homogeneous degree $\alpha_{\ell}$ depends only on the components of $p, q, r, \ldots$ of degree not higher than $\alpha_{\ell}$.

More precisely, formula (7) extends formula (6) to the case of the product of $d \geq 2$ factors.

$$
\begin{aligned}
& \underbrace{p \cdot q \cdot r \cdot \ldots}_{d \text { factors }}=\left(p^{1}+q^{1}+r^{1}+\ldots,\right. \\
& p^{2}+q^{2}+r^{2}+\ldots+\tilde{\mathcal{Q}}^{2}(d ; p, q, r, \ldots), \ldots, \\
& \left.p^{\kappa}+q^{\kappa}+r^{\kappa}+\ldots+\tilde{\mathcal{Q}}^{\kappa}(d ; p, q, r, \ldots)\right)
\end{aligned}
$$

where each $\tilde{\mathcal{Q}}^{i}(d ; \cdot)$, for $2 \leq i \leq \kappa$, is a vector valued polynomial of homogeneous degree $i$ depending only on the components of $p, q, r, \ldots$ in the first $i-1$ strata, i.e. depending only on

$$
p_{1}, \ldots, p_{m_{i-1}}, q_{1}, \ldots, q_{m_{i-1}}, r_{1}, \ldots, r_{m_{i-1}}, \ldots
$$

(observe that $\left.\tilde{\mathcal{Q}}^{i}(2 ; \cdot)=\mathcal{Q}^{i}(\cdot)\right)$. Where in components we can write

$$
\tilde{\mathcal{Q}}^{i}(d ; p, q, r, \ldots)=\left(\tilde{\mathcal{Q}}_{m_{i-1}+1}(d ; p, q, r, \ldots), \ldots, \tilde{\mathcal{Q}}_{m_{i}}(d ; p, q, r, \ldots)\right)
$$

and each $\tilde{\mathcal{Q}}_{\ell}=\tilde{\mathcal{Q}}_{\ell}(d ; p, q, r, \ldots)$, for $m_{i-1}+1 \leq \ell \leq m_{i}$, is a linear combination of homogeneous monomials of the form

$$
\prod_{j=1}^{m_{i-1}} p_{j}^{\beta_{j}^{1}} q_{j}^{\beta_{j}^{2}} r_{j}^{\beta_{j}^{3}} \cdots
$$

where, for $1 \leq k \leq d$, each $\beta^{k}=\left(\beta_{1}^{k}, \ldots, \beta_{m_{i-1}}^{k}\right)$ is a multi index of non negative integers such that

$$
\sum_{j=1}^{m_{i-1}}\left(\sum_{k=1}^{d} \beta_{j}^{k}\right) \alpha_{j}=i=\alpha_{\ell} .
$$

In order to prove the assertion, let us notice first that equation (7) can be written also as

$$
\begin{aligned}
& (p \cdot q \cdot r \cdot \ldots)_{\ell} \\
& \quad=p_{\ell}+q_{\ell}+r_{\ell}+\ldots+\tilde{\mathcal{Q}}_{\ell}\left(d ; p_{1}, \ldots, p_{m_{i-1}}, q_{1}, \ldots, q_{m_{i-1}}, r_{1}, \ldots, r_{m_{i-1}}, \ldots\right)
\end{aligned}
$$

for $m_{i-1}+1 \leq \ell \leq m_{i}$ and for $1 \leq i \leq \kappa$. 
Both (7) and (10) can be proved by induction on the number of factors $d$. Indeed, suppose the assertion holds for $d$ factors and let us prove it for $d+1$ factors. We can write the product of $d+1$ factors as

$$
p \cdot \underbrace{q \cdot r \cdot \ldots}_{d \text { factors }}=p \cdot z
$$

By (4), if $m_{i-1}+1 \leq \ell \leq m_{i}$, then

$$
(p \cdot q \cdot r \cdot \ldots)_{\ell}=p_{\ell}+z_{\ell}+\mathcal{Q}_{\ell}\left(p_{1}, \ldots, p_{m_{i-1}}, z_{1}, \ldots, z_{m_{i-1}}\right),
$$

where the polynomials $Q_{\ell}\left(p_{1}, \ldots, p_{m_{i-1}}, z_{1}, \ldots, z_{m_{i-1}}\right)$ are linear combinations of homogeneous monomials of the form

$$
\prod_{j=1}^{m_{i-1}} p_{j}^{\beta_{j}^{1}} z_{j}^{\eta_{j}}, \quad \text { with } \quad \sum_{j=1}^{m_{i-1}}\left(\beta_{j}^{1}+\eta_{j}\right) \alpha_{j}=\alpha_{\ell} .
$$

By the induction assumption, each $z_{j}$ is the linear combination of homogeneous monomials of degree $\alpha_{j}$ and of the form

$$
\prod_{h=1}^{j} q_{h}^{\gamma_{h}^{2}} r_{h}^{\gamma_{h}^{3}} \ldots \quad \text { with } \quad \sum_{h=1}^{j}\left(\sum_{k=2}^{d+1} \gamma_{h}^{k}\right) \alpha_{h}=\alpha_{j} .
$$

Hence also $\prod_{j=1}^{m_{i-1}} p_{j}^{\beta_{j}^{1}} z_{j}^{\eta_{j}}$ is the linear combination of homogeneous monomials of total homogeneous degree $\alpha_{\ell}$, since each $z_{j}$, that is a variable with homogeneity $\alpha_{j}$, is replaced by a linear combination of homogeneous monomials with the same homogeneity $\alpha_{j}$. More formally

$$
\begin{aligned}
\prod_{j=1}^{m_{i-1}} p_{j}^{\beta_{j}^{1}} z_{j}^{\eta_{j}} & =\prod_{j=1}^{m_{i-1}} p_{j}^{\beta_{j}^{1}}\left(\text { linear combination of } \prod_{h=1}^{j} q_{h}^{\gamma_{h}^{2}} r_{h}^{\gamma_{h}^{3}} \ldots\right)^{\eta_{j}} \\
& =\text { linear combination of } \prod_{j=1}^{m_{i-1}} p_{j}^{\beta_{j}^{1}} \prod_{h=1}^{j} q_{h}^{\beta_{h}^{2}} r_{h}^{\beta_{h}^{3}} \ldots
\end{aligned}
$$

with, by (11),

$$
\sum_{h=1}^{j}\left(\sum_{k=2}^{d+1} \beta_{h}^{k}\right) \alpha_{h}=\alpha_{j} \eta_{j}, \quad \text { and } \quad \sum_{j=1}^{m_{i-1}}\left(\sum_{\ell=1}^{d} \beta_{j}^{\ell}\right) \alpha_{j}=\alpha_{\ell} .
$$

This concludes the proof of (7) and of (10).

Definition 1. An absolutely continuous curve $\gamma:[0, T] \rightarrow \mathbb{G}$ is a sub-unit curve with respect to $X_{1}, \ldots, X_{m_{1}}$ if there exist measurable real functions $c_{1}(s), \ldots, c_{m_{1}}(s), s \in[0, T]$ such that $\sum_{j} c_{j}^{2} \leq 1$ and

$$
\dot{\gamma}(s)=\sum_{j=1}^{m_{1}} c_{j}(s) X_{j}(\gamma(s)), \quad \text { for a.e. } s \in[0, T] .
$$

Definition 2. If $p, q \in \mathbb{G}$, we define their Carnot-Carathéodory distance as

$$
d_{c}(p, q):=\inf \{T>0: \text { there exists a sub-unit curve } \gamma \text { with } \gamma(0)=p, \gamma(T)=q\} .
$$

By Chow's Theorem, the set of sub-unit curves joining $p$ and $q$ is not empty, furthermore $d_{c}$ is a distance on $\mathbb{G}$ that induces the Euclidean topology (see chapter 19 in [9] or Theorem 1.6.2 in [35]).

Definition 3. A nonnegative function $p \rightarrow\|p\|$ on $\mathbb{G}$ is said an homogeneous norm if

i) $\|p\|=0$ if and only if $p=e$;

ii) $\left\|\delta_{\lambda} p\right\|=\lambda\|p\|$ for all $p \in \mathbb{G}$ and $\lambda>0$; 
iii) $\|p \cdot q\| \leq\|p\|+\|q\|$.

Given any homogeneous norm $\|\cdot\|$, it is possible to define a distance in $\mathbb{G}$ as

$$
d(p, q)=d\left(q^{-1} \cdot p, 0\right)=\left\|q^{-1} \cdot p\right\| \quad \text { for all } p, q \in \mathbb{G} .
$$

The distance $d$ in (14) is comparable with the Carnot-Carathéodory distance of $\mathbb{G}$ and

$$
d(g \cdot p, g \cdot q)=d(p, q), \quad d\left(\delta_{\lambda}(p), \delta_{\lambda}(q)\right)=\lambda d(p, q)
$$

for all $p, q, g \in \mathbb{G}$ and all $\lambda>0$.

A convenient homogeneous norm, and the one used here, is described in [23, Theorem 5.1]. It is defined as

$$
d_{\infty}(p, 0):=\|p\|:=\max _{j=1, \ldots, \kappa}\left\{\varepsilon_{j}\left\|p^{j}\right\|_{\mathbb{R}^{n_{j}}}^{1 / j}\right\}, \quad \text { for all } p=\left(p^{1}, \ldots, p^{\kappa}\right) \in \mathbb{G},
$$

where $\varepsilon_{1}=1$, and $\varepsilon_{2}, \ldots \varepsilon_{\kappa} \in(0,1]$ are suitable positive constants depending on $\mathbb{G}$.

For $r>0$ and $p \in \mathbb{G}$, we denote by $U_{c}(p, r)$ and $B_{c}(p, r)$, respectively, the open and closed balls associated with the Carnot-Carathéodory distance $d_{c}$, and with $U(p, r), B(p, r)$ the ones associated with $d$ as in (14).

Definition 4. The integer $Q=\sum_{j=1}^{n} \alpha_{j}=\sum_{i=1}^{\kappa} i \operatorname{dim} V_{i}$ is the homogeneous dimension of $\mathbb{G}$. $Q$ is also the Hausdorff dimension of $\mathbb{R}^{n}$ with respect to $d_{c}$ (see [34]).

Proposition 2.1.2. The $n$-dimensional Lebesgue measure $\mathcal{L}^{n}$ is the Haar measure of the group $\mathbb{G}$ (see [42]). Therefore if $E \subset \mathbb{R}^{n}$ is measurable, then $\mathcal{L}^{n}(g \cdot E)=\mathcal{L}^{n}(E)$ for every $g \in \mathbb{G}$. Moreover, if $\lambda>0$ then $\mathcal{L}^{n}\left(\delta_{\lambda}(E)\right)=\lambda^{Q} \mathcal{L}^{n}(E)$. We note that

$$
\mathcal{L}^{n}\left(U_{c}(p, r)\right)=r^{Q} \mathcal{L}^{n}\left(U_{c}(p, 1)\right)=r^{Q} \mathcal{L}^{n}\left(U_{c}(0,1)\right) .
$$

Using the distances $d_{c}$ or $d$ the family of Hausdorff measures or spherical Hausdorff measures are obtained following Carathéodory's construction (see [17, Section 2.10.2.]). Precisely, if $m \geq 0$ and $\mathcal{A} \subset \mathbb{G}$, the (intrinsic spherical) Hausdorff measure $\mathcal{S}_{d}^{m}$ is,

$$
\mathcal{S}_{d}^{m}(\mathcal{A}):=\lim _{\delta \rightarrow 0} \mathcal{S}_{d, \delta}^{m}(\mathcal{A})
$$

where $\mathcal{S}_{d, \delta}^{m}(\mathcal{A})=\inf \left\{\sum_{i} r_{i}^{m}: \mathcal{A} \subset \bigcup_{i} B\left(p_{i}, r_{i}\right), r_{i} \leq \delta\right\}$. The Hausdorff measures $\mathcal{H}_{d}^{m}$ are defined analogously.

Translation invariance and dilation homogeneity of Hausdorff measures follow from (15) and, for $\mathcal{A} \subseteq \mathbb{G}, p \in \mathbb{G}$ and $r \in[0, \infty)$,

$$
\mathcal{S}_{d}^{m}(p \cdot \mathcal{A})=\mathcal{S}_{d}^{m}(\mathcal{A}) \quad \text { and } \quad \mathcal{S}_{d}^{m}\left(\delta_{r} \mathcal{A}\right)=r^{m} \mathcal{S}_{d}^{m}(\mathcal{A}) .
$$

A homogeneous subgroup of a Carnot group $\mathbb{G}$ (see $[41,5.2 .4]$ ) is a Lie subgroup $\mathbb{H}$ such that $\delta_{\lambda} g \in \mathbb{H}$, for all $g \in \mathbb{H}$ and for all $\lambda>0$. Homogeneous subgroups are linear subspaces of $\mathbb{G}$, when $\mathbb{G}$ is identified with $\mathbb{R}^{n}$ with exponential coordinates.

Remark 2.1.3. An homogeneous subgroup $\mathbb{H}$ is stratified, that is $\mathbb{H}=\mathbb{H}^{1} \oplus \cdots \oplus \mathbb{H}^{\kappa}$, where $\mathbb{H}^{i} \subset \mathbb{G}^{i}$ and $\mathbb{H}^{i}$ is a linear subspace of $\mathbb{G}^{i}$. If we denote by $\mathfrak{h}$ the Lie algebra of $\mathbb{H}$, this follows once we prove that

$$
\mathfrak{h}=\oplus_{p=1}^{\kappa} \mathfrak{h}_{p},
$$

where $\mathfrak{h}_{p}=\mathfrak{h} \cap \mathfrak{g}_{p}$. Indeed, if $v \in \mathfrak{h}$, we can write $v=\sum_{p} v_{p}$, with $v_{p} \in \mathfrak{g}_{p}, p=1, \ldots, \kappa$. Thus (17) follows if we show that

$$
v_{p} \in \mathfrak{h} \quad \text { for all } p=1, \ldots, \kappa \text {. }
$$


To this end, we remind that $\mathfrak{h}$ is a vector space and, in addition, it is homogeneous with respect to group dilations. Hence, for $\lambda>0$,

$$
\frac{1}{\lambda} \delta_{\lambda} v:=\frac{1}{\lambda} \sum_{p} \lambda^{p} v_{p}=v_{1}+\sum_{p \geq 2} \lambda^{p-1} v_{p} \in \mathfrak{h} .
$$

But $\frac{1}{\lambda} \delta_{\lambda} v$ is bounded, and hence, if we choose $\lambda=\lambda_{n}$, with $\lambda_{n} \rightarrow 0$ as $n \rightarrow \infty$, we can assume $\left(\frac{1}{\lambda_{n}} \delta_{\lambda_{n}} v\right)_{n}$ has a limit in $\mathfrak{h}$. Thus, we can conclude that $v_{1} \in \mathfrak{h}$. We can repeat now the argument replacing $v$ by $v-v_{1} \in \mathfrak{h}$, and we write

$$
\frac{1}{\lambda^{2}} \delta_{\lambda}\left(v-v_{1}\right)=v_{2}+\sum_{p \geq 3} \lambda^{p-2} v_{p} \in \mathfrak{h},
$$

obtaining eventually that $v_{2} \in \mathfrak{h}$. Iterating this argument, we get (18) and therefore (17).

The topological dimension of a (sub)group is the dimension of its Lie algebra. The metric dimension of a subset is its Hausdorff dimension with respect to the Carnot-Carathéodory distance $d_{c}$ in $\mathbb{G}$. The metric dimension of a homogeneous subgroup is an integer usually larger than its topological dimension (see [34]).

\subsection{Complementary subgroups and graphs.}

2.2.1. Complementary subgroups. From now on $\mathbb{G}$ will always be a homogeneous stratified group, identified with $\mathbb{R}^{n}$ with exponential coordinates.

Definition 5. Let $\mathbb{M}, \mathbb{H}$ be homogeneous subgroups of $\mathbb{G}$. We say that $\mathbb{M}, \mathbb{H}$ are complementary subgroups in $\mathbb{G}$, if $\mathbb{M} \cap \mathbb{H}=\{e\}$ and if

$$
\mathbb{G}=\mathbb{M} \cdot \mathbb{H} \text {, }
$$

that is for each $g \in \mathbb{G}$, there are $m \in \mathbb{M}$ and $h \in \mathbb{H}$ such that $g=m \cdot h$.

If $\mathbb{M}, \mathbb{H}$ are complementary subgroups of $\mathbb{G}$ and one of them is a normal subgroup then $\mathbb{G}$ is said to be the semi-direct product of $\mathbb{M}$ and $\mathbb{H}$. If both $\mathbb{M}$ and $\mathbb{H}$ are normal subgroups then $\mathbb{G}$ is said to be the direct product of $\mathbb{M}$ and $\mathbb{H}$.

By elementary facts in group theory (see e.g. [30, Lemma 2.8]) if $\mathbb{M}, \mathbb{H}$ are complementary subgroups in $\mathbb{G}$, so that $\mathbb{G}=\mathbb{M} \cdot \mathbb{H}$, then it is also true that

$$
\mathbb{G}=\mathbb{H} \cdot \mathbb{M}
$$

that is, each $g \in \mathbb{G}$ can be written - in a unique way - as $g=\bar{h} \bar{m}$, with $\bar{m} \in \mathbb{M}, \bar{h} \in \mathbb{H}$. Rephrased differently, if $\mathbb{M}, \mathbb{H}$ are complementary subgroups in $\mathbb{G}$, also $\mathbb{H}, \mathbb{M}$ are complementary subgroups in $\mathbb{G}$.

Remark 2.2.1. If $\mathbb{G}$ is stratified and $\mathbb{M}, \mathbb{H}$ are complementary subgroups in $\mathbb{G}$ then they are stratified and also $\mathbb{G}^{i}=\mathbb{M}^{i} \oplus \mathbb{H}^{i}$, for $i=1, \ldots, \kappa$.

Example 2.2.2. Let $\mathbb{G}$ be the Heisenberg group $\mathbb{H}^{n}$. In this case $\mathfrak{g}=\mathfrak{g}_{1} \oplus \mathfrak{g}_{2}$, with $\operatorname{dim} \mathfrak{g}_{1}=2 n$ and $\operatorname{dim} \mathfrak{g}_{2}=1$. Then all the possible couples of complementary subgroups of $\mathbb{H}^{n}$ contain a horizontal subgroup $\mathbb{V}$ of dimension $k \leq n$, isomorphic and isometric to $\mathbb{R}^{k}$ and a normal subgroup $\mathbb{W}$ of dimension $2 n+1-k$, containing the center $\mathbb{T}$. Moreover $\mathbb{W}^{1} \oplus \mathbb{V}=\mathbb{G}^{1}$. Indeed a sub algebra of $\mathfrak{g}$ either contains $\mathfrak{g}_{2}$ (and hence is an ideal) or is contained in $\mathfrak{g}_{1}$ (and hence is Abelian).

Similar splittings exist in a general Carnot group $\mathbb{G}$ (but usually are not the only possible splittings: see Example 2.2.3 below). Indeed, choose any horizontal homogeneous subgroup $\mathbb{H}=$ $\mathbb{H}^{1} \subset \mathbb{G}^{1}$ and a subgroup $\mathbb{M}=\mathbb{M}^{1} \oplus \cdots \oplus \mathbb{M}^{\kappa}$ such that: $\mathbb{H} \oplus \mathbb{M}^{1}=\mathbb{G}^{1}$, and $\mathbb{G}^{j}=\mathbb{M}^{j}$ for all $2 \leq j \leq \kappa$. Then $\mathbb{M}$ and $\mathbb{H}$ are complementary subgroups in $\mathbb{G}$ and the product $\mathbb{G}=\mathbb{M} \cdot \mathbb{H}$ is semidirect because $\mathbb{M}$ is a normal subgroup. 
Example 2.2.3. The Engels group is $\mathbb{E}=\left(\mathbb{R}^{4}, \cdot, \delta_{\lambda}\right)$, were the group law is defined as

$$
\left(\begin{array}{l}
x_{1} \\
x_{2} \\
x_{3} \\
x_{4}
\end{array}\right) \cdot\left(\begin{array}{l}
y_{1} \\
y_{2} \\
y_{3} \\
y_{4}
\end{array}\right)=\left(\begin{array}{c}
x_{1}+y_{1} \\
x_{2}+y_{2} \\
x_{4}+y_{4}+\left[\left(x_{1} y_{3}-x_{3} y_{1}\right)+\left(x_{2} y_{3}-x_{3} y_{2}\right)\right] / 2 \\
+\left(x_{1}-y_{1}+x_{2}-y_{2}\right)\left(x_{1} y_{2}-x_{2} y_{1}\right) / 12
\end{array}\right)
$$

and the family of dilation is

$$
\delta_{\lambda}\left(x_{1}, x_{2}, x_{3}, x_{4}\right)=\left(\lambda x_{1}, \lambda x_{2}, \lambda^{2} x_{3}, \lambda^{3} x_{4}\right) .
$$

Inside Engels group there are two families of complementary subgroups. The first one is formed by 1-dimensional horizontal subgroups and 3-dimensional subgroups containing all the vertical directions. This family gives a semidirect splitting of $\mathbb{E}$. The second family is formed by 2dimensional subgroups that are not normal subgroups. All the computations can be easily done directly, better using a symbolic computation program.

The homogeneous subgroups

$$
\mathbb{M}_{\alpha, \beta}:=\{(\alpha t, \beta t, 0,0): t \in \mathbb{R}\}, \quad \mathbb{N}_{\gamma, \delta}:=\left\{\left(\gamma t, \delta t, x_{3}, x_{4}\right): t, x_{3}, x_{4} \in \mathbb{R}\right\},
$$

are complementary subgroups in $\mathbb{E}$, provided that $\alpha \delta-\beta \gamma \neq 0$. Moreover $\mathbb{N}_{\gamma, \delta}$ is a normal subgroup, hence $\mathbb{E}$ is the semidirect product of $\mathbb{M}_{\alpha, \beta}$ and $\mathbb{N}_{\gamma, \delta}$.

The second family, for $\alpha+\beta \neq 0$, is given by

$$
\mathbb{K}:=\left\{\left(x_{1},-x_{1}, x_{3}, 0\right): x_{1}, x_{3} \in \mathbb{R}\right\} \quad \mathbb{H}_{\alpha, \beta}:=\left\{\left(\alpha t, \beta t, 0, x_{4}\right): t, x_{4} \in \mathbb{R}\right\} .
$$

One can compute directly that $\mathbb{K}$ and $\mathbb{H}_{\alpha, \beta}$ are complementary subgroups in $\mathbb{E}$ and that neither $\mathbb{K}$ nor $\mathbb{H}_{\alpha, \beta}$ are normal subgroups. Hence $\mathbb{E}=\mathbb{K} \cdot \mathbb{H}_{\alpha, \beta}$, but the product is not a semidirect product.

Example 2.2.4. Let us hint here some relations between the Rumin's complex of intrinsic differential forms in a Carnot group $\mathbb{G}$ and the existence of complementary subgroups in $\mathbb{G}$. Necessarily, we will be very sketchy here. For further details we refer the reader to [38], [39], [10], [27].

Let $\mathbb{G}$ be a Carnot group, and let $\mathfrak{g}$ be its Lie algebra. The dual space of $\mathfrak{g}$ is denoted by $\bigwedge^{1} \mathfrak{g}$. The basis of $\bigwedge^{1} \mathfrak{g}$, dual of the basis $X_{1}, \cdots, X_{n}$, is the family of covectors $\left\{\theta_{1}, \cdots, \theta_{n}\right\}$. We indicate by $\langle\cdot, \cdot\rangle$ also the inner product in $\Lambda^{1} \mathfrak{g}$ that makes $\theta_{1}, \cdots, \theta_{n}$ an orthonormal basis. We point out that, except for the trivial case of the commutative group $\mathbb{R}^{n}$, the forms $\theta_{1}, \cdots, \theta_{n}$ may have polynomial (hence variable) coefficients.

Following Federer (see [17] 1.3), the exterior algebras of $\mathfrak{g}$ and of $\Lambda^{1} \mathfrak{g}$ are the graded algebras indicated as $\bigwedge_{*} \mathfrak{g}=\bigoplus_{h=0}^{n} \bigwedge_{h} \mathfrak{g}$ and $\bigwedge^{*} \mathfrak{g}=\bigoplus_{h=0}^{n} \bigwedge^{h} \mathfrak{g}$ where $\bigwedge_{0} \mathfrak{g}=\bigwedge^{0} \mathfrak{g}=\mathbb{R}$ and, for $1 \leq h \leq n$,

$$
\begin{aligned}
& \bigwedge_{h} \mathfrak{g}:=\operatorname{span}\left\{X_{i_{1}} \wedge \cdots \wedge X_{i_{h}}: 1 \leq i_{1}<\cdots<i_{h} \leq n\right\} \\
& \bigwedge^{h} \mathfrak{g}:=\operatorname{span}\left\{\theta_{i_{1}} \wedge \cdots \wedge \theta_{i_{h}}: 1 \leq i_{1}<\cdots<i_{h} \leq n\right\} .
\end{aligned}
$$

The elements of $\bigwedge_{h} \mathfrak{g}$ and $\bigwedge^{h} \mathfrak{g}$ are called h-vectors and $h$-covectors, respectively. As usual $\bigwedge_{h} \mathfrak{g}$ and $\Lambda^{h} \mathfrak{g}$ define a family of fiber bundle over $\mathbb{G}$ that we still denote as $\Lambda_{h} \mathfrak{g}$ and $\Lambda^{h} \mathfrak{g}$. We denote by $\Omega_{h}$ and $\Omega^{h}$ the spaces of sections of $\Lambda_{h} \mathfrak{g}$ and $\Lambda^{h} \mathfrak{g}$. We refer to elements of $\Omega_{h}$ as fields of $h$-vectors and to elements of $\Omega^{h}$ as $h$-forms and to $\left(\Omega^{*}, d\right)$ as to the De Rham complex.

The dual space $\Lambda^{1}\left(\bigwedge_{h} \mathfrak{g}\right)$ of $\bigwedge_{h} \mathfrak{g}$ can be naturally identified with $\Lambda^{h} \mathfrak{g}$. If $v \in \bigwedge_{h} \mathfrak{g}$ we define $v^{\natural} \in \Lambda^{h} \mathfrak{g}$ by the identity $\left\langle v^{\natural} \mid w\right\rangle:=\langle v, w\rangle$, and analogously we define $\varphi^{\natural} \in \bigwedge_{h} \mathfrak{g}$ for $\varphi \in \Lambda^{h} \mathfrak{g}$.

The inner product $\langle\cdot, \cdot\rangle$ extends canonically to $\bigwedge_{h} \mathfrak{g}$ and to $\bigwedge^{h} \mathfrak{g}$ making the bases $\left\{X_{i_{1}} \wedge \cdots \wedge X_{i_{h}}\right\}$ and $\left\{\theta_{i_{1}} \wedge \cdots \wedge \theta_{i_{h}}\right\}$ orthonormal. 
Definition 6. If $\alpha \in \bigwedge^{1} \mathfrak{g}, \alpha \neq 0$, we say that $\alpha$ has pure weight $k$, and we write $w(\alpha)=k$, if its dual vector $\alpha^{\natural}$ is in $\mathfrak{g}_{k}$. More generally, if $\alpha \in \Lambda^{h} \mathfrak{g}$, we say that $\alpha$ has pure weight $k$ if $\alpha$ is a linear combination of covectors $\theta_{i_{1}} \wedge \cdots \wedge \theta_{i_{h}}$ with $w\left(\theta_{i_{1}}\right)+\cdots+w\left(\theta_{i_{h}}\right)=k$.

If $\alpha, \beta \in \bigwedge^{h} \mathfrak{g}$ and $w(\alpha) \neq w(\beta)$, then $\langle\alpha, \beta\rangle=0$, and we have (see [10], formula (16))

$$
\bigwedge^{h} \mathfrak{g}=\bigoplus_{p=M_{h}^{\min }}^{M_{h}^{\max }} \bigwedge^{h, p} \mathfrak{g},
$$

where $\bigwedge^{h, p} \mathfrak{g}$ is the linear span of the $h$-covectors of weight $p$ and $M_{h}^{\min }, M_{h}^{\max }$ are respectively the smallest and the largest weight of $h$-covectors.

We denote also by $\Omega^{h, p}$ the vector space of all smooth $h$-forms in $\mathbb{G}$ of pure weight $p$, i.e. the space of all smooth sections of $\bigwedge^{h, p} \mathfrak{g}$. We have

$$
\Omega^{h}=\bigoplus_{p=M_{h}^{\min }}^{M_{h}^{\max }} \Omega^{h, p} .
$$

The filtration (19) induces a decomposition

$$
d \alpha=d_{0} \alpha+d_{1} \alpha+\cdots+d_{\kappa} \alpha,
$$

of the exterior differential $d: \Omega^{h} \rightarrow \Omega^{h+1}$, where $d_{0}$ does not increase the weight, and $d_{i}$ increases the weight by $i$ for $i=1, \ldots, \kappa$. In particular, $d_{0}$ is an algebraic operator.

Lemma 2.2.5. $d_{0}^{2}=0$, i.e. $\left(\Omega^{*}, d_{0}\right)$ is a complex. Moreover, if $\alpha \in \Omega^{h}$ is left-invariant, then

$$
\begin{gathered}
d \alpha=d_{0} \alpha \\
d_{0} \alpha \text { is left-invariant; } \\
\text { if } d \alpha=d_{0} \alpha \neq 0 \text {, then the weight of } d_{0} \alpha \text { equals the weight of } \alpha .
\end{gathered}
$$

The following definitions are due to M. Rumin ([38], [39]).

Definition 7. If $0 \leq h \leq n$ we set

$$
E_{0}^{h}:=\operatorname{ker} d_{0} \cap\left(\operatorname{Im} d_{0}\right)^{\perp}=\operatorname{ker} d_{0} \cap \operatorname{ker}\left(d_{0} *\right),
$$

where $*$ denotes the Hodge duality operator associated with the scalar product in $\mathfrak{g}$ and the volume form $d V:=\theta_{1} \wedge \cdots \wedge \theta_{n}$.

The elements of $E_{0}^{h}$ are denoted as intrinsic $h$-forms on $\mathbb{G}$. Since the construction of $E_{0}^{h}$ is left invariant, this space of forms can be seen as the space of sections of a fiber subbundle of $\Lambda^{h} \mathfrak{g}$, generated by left translation and still denoted by $E_{0}^{h}$ (the bundle of the intrinsic covectors). In particular $E_{0}^{h}$ inherits from $\bigwedge^{h} \mathfrak{g}$ the scalar product on the fibers.

We denote by $N_{h}^{\min }$ and $N_{h}^{\max }$ the minimum and the maximum, respectively, of the weights of forms in $E_{0}^{h}$. If we set $E_{0}^{h, p}:=E_{0}^{h} \cap \Omega^{h, p}$, then

$$
E_{0}^{h}=\bigoplus_{p=N_{h}^{\min }}^{N_{h}^{\max }} E_{0}^{h, p} .
$$

Indeed, if $\alpha \in E_{0}^{h}$, by (19), we can write $\alpha=\sum_{p=N_{h}^{\min }}^{N_{h}^{\max }} \alpha_{p}$, with $\alpha_{p} \in \Omega^{h, p}$ for all $p$. The assertion follows by proving that $\alpha_{p} \in E_{0}^{h}$. Indeed, by definition, $0=d_{0} \alpha=\sum_{p=N_{h}^{\min }}^{N_{\max }^{\max }} d_{0} \alpha_{p}$. But the weight of $d_{0} \alpha_{p}$ is different from that of $d_{0} \alpha_{q}$ for $p \neq q$, and hence the $d_{0} \alpha_{p}$ 's are linear independent and therefore they are all 0 . The same argument can be repeated for $* \alpha$, and the assertion follows. 
The following result shows that a pair of non-parallel intrinsic simple covectors $\xi \in E_{0}^{h}$ and $\omega \in E_{0}^{n-h}$ naturally define a couple of complementary subgroups as in Definition 5 . Following the notations of [29], p.90, if $X$ is a vector field, we denote by $i(X)$ the exterior product.

Proposition 2.2.6. Let $\mathbb{G}$ be a Lie group of dimension $n$, and denote by $\mathfrak{g}$ the Lie algebra of the left invariant vector fields on $\mathbb{G}$. Without loss of generality, we may assume that a scalar product is fixed in $\mathfrak{g}$.

Let now $\mathfrak{h}$ be a Lie subalgebra of $\mathfrak{g}$ of dimension $h$, and let $Z_{1}, \ldots, Z_{n-h}$ be a basis of $\mathfrak{h}^{\perp}$. If we set $\omega_{i}:=Z_{i}^{\natural}$ for $i=1, \ldots, n-h$ and $\omega:=\omega_{1} \wedge \cdots \wedge \omega_{n-h}$, then

i) $\mathfrak{h}=\{X \in \mathfrak{g}: i(X) \omega=0\}=\left\{X \in \mathfrak{g}: * \omega \wedge X^{\natural}=0\right\}$.

ii) there exists $\beta \in \Lambda^{1} \mathfrak{g}$ such that $d \omega=\beta \wedge \omega$.

Reciprocally, if $\omega:=\omega_{1} \wedge \cdots \wedge \omega_{n-h} \in \bigwedge^{n-h} \mathfrak{g}$ is a simple left invariant form such that $d \omega=\beta \wedge \omega$ for some $\beta \in \bigwedge^{1} \mathfrak{g}$, then

iii) $\mathfrak{h}:=\{X \in \mathfrak{g}: i(X) \omega=0\}$ is a Lie subalgebra of $\mathfrak{h}$.

Proof. Assertion i) is well known (see e.g. [17], Section 1.6, [16], Section 2.3). On the other hand, assertions ii) and iii) follow by Frobenius theorem (see, e.g., [1], Theorem 7.4.24).

Theorem 2.2.7. If $1 \leq h<n, \xi \in E_{0}^{h}$ and $\omega \in E_{0}^{n-h}$ are simple covectors such that

$$
\xi \wedge \omega \neq 0,
$$

we set

$$
\mathfrak{m}:=\{X \in \mathfrak{g}: i(X) \xi=0\}, \quad \mathfrak{h}:=\{X \in \mathfrak{g}: i(X) \omega=0\} .
$$

Then both $\mathfrak{m}$ and $\mathfrak{h}$ are Lie subalgebras of $\mathfrak{g}$. Moreover $\operatorname{dim} \mathfrak{m}=n-h, \operatorname{dim} \mathfrak{h}=h$ and $\mathfrak{g}=\mathfrak{m} \oplus \mathfrak{h}$. If, in addition, $\xi=\xi_{1} \wedge \cdots \wedge \xi_{h}, \omega=\omega_{1} \wedge \cdots \wedge \omega_{n-h}$, where all the $\xi_{i}$ 's and the $\omega_{i}$ have pure weights $p_{i}$ and $q_{i}$, respectively, then both $\mathfrak{m}$ and $\mathfrak{h}$ are homogeneous Lie subalgebras of $\mathfrak{g}$. Thus, if we set

$$
\mathbb{M}:=\exp (\mathfrak{m}) \quad \text { and } \quad \mathbb{H}:=\exp (\mathfrak{h}),
$$

then $\mathbb{M}$ and $\mathbb{G}$ are complementary subgroups. In particular, since $* E_{0}^{h}=E_{0}^{n-h}$, if $\xi \in E_{0}^{h}$, we can choose $\omega:=* \xi$. In this case, $\mathfrak{m}$ and $\mathfrak{h}$ are orthogonal.

Reciprocally, suppose $\mathfrak{m}$ and $\mathfrak{h}$ are homogeneous Lie subalgebras of $\mathfrak{g}$ such that $\operatorname{dim} \mathfrak{m}=n-h$, $\operatorname{dim} \mathfrak{h}=h$, and $\mathfrak{g}=\mathfrak{m} \oplus \mathfrak{h}$. Then there exist a scalar product $\langle\cdot, \cdot\rangle$ in $\mathfrak{g}, \xi \in E_{0}^{h}$ and $\omega \in E_{0}^{n-h}$ such that $\xi \wedge \omega \neq 0$ and

$$
\mathfrak{m}:=\{X \in \mathfrak{g}: i(X) \xi=0\}, \quad \mathfrak{h}:=\{X \in \mathfrak{g}: i(X) \omega=0\} .
$$

Remark 2.2.8. We remind that, as discussed in [38] and [27], Remark 3.13, our definition of Rumin's classes depends on the scalar product in $\mathfrak{g}$.

Proof of Theorem 2.2.7. By Proposition 2.2.6, both $\mathfrak{m}$ and $\mathfrak{h}$ are Lie subalgebras, since $d \xi=0$ and $d \omega=0$. In addition, $\operatorname{dim} \mathfrak{m}=n-h, \operatorname{dim} \mathfrak{h}=h$ and $\mathfrak{g}=\mathfrak{m} \oplus \mathfrak{h}$ and hence $\mathfrak{g}=\mathfrak{m} \oplus \mathfrak{h}$, since $\mathfrak{m} \cap \mathfrak{h}=\{0\}$.

Suppose now, for instance, $\xi=\xi_{1} \wedge \cdots \wedge \xi_{h}$, where all the $\xi_{i}$ 's have pure weight $p_{i}, i=1, \ldots, h$. Then $\xi$ has weight $p:=p_{1}+\cdots+p_{h}$. Take $X \in \mathfrak{m}$; we can write

$$
X=\sum_{\ell=1}^{\kappa} \mu_{\ell} v_{\ell}, \quad \text { with } v_{\ell} \in \mathfrak{g}_{\ell} .
$$


By identity 6 (ii) of [29], p.90, if we set $\hat{\xi}_{j}:=\xi_{1} \wedge \cdots \wedge \xi_{j-1} \wedge \xi_{j+1} \wedge \cdots \wedge \xi_{h}$, we can write

$$
\begin{aligned}
0=i(X) \xi & =\sum_{1 \leq j \leq h}(-1)^{j+1}\left(i(X) \xi_{j}\right) \hat{\xi}_{j}=\sum_{1 \leq j \leq h}(-1)^{j+1}\left\langle\xi_{j} \mid X\right\rangle \hat{\xi}_{j} \\
& =\sum_{\ell=1}^{\kappa} \sum_{1 \leq j \leq h}(-1)^{j+1} \mu_{\ell}\left\langle\xi_{j} \mid v_{\ell}\right\rangle \hat{\xi}_{j}=\sum_{i=1}^{\kappa} \sum_{\ell=1}^{\kappa} \sum_{p_{j}=i}(-1)^{j+1} \mu_{\ell}\left\langle\xi_{j} \mid v_{\ell}\right\rangle \hat{\xi}_{j} \\
& =\sum_{i=1}^{\kappa} \sum_{p_{j}=i}(-1)^{j+1} \mu_{i}\left\langle\xi_{j} \mid v_{i}\right\rangle \hat{\xi}_{j},
\end{aligned}
$$

since $\left\langle\xi_{j} \mid v_{\ell}\right\rangle=0$ if $\ell$ does not equal the weight of $\xi_{j}$. Notice now that, if $p_{j}=i$, then the weight of $\hat{\xi}_{j}=p-i$, and then the $\hat{\xi}_{j}$ 's are orthogonal when the $p_{j}$ 's are different. It follows that

$$
\sum_{p_{j}=i}(-1)^{j+1} \mu_{i}\left\langle\xi_{j} \mid v_{i}\right\rangle \hat{\xi}_{j}=0 \quad \text { for } i=1, \ldots, \kappa .
$$

If now $\lambda>0$, arguing as in (20), we get

$$
\begin{aligned}
i\left(\delta_{\lambda} X\right) \xi & =\sum_{1 \leq j \leq h}(-1)^{j+1}\left(i\left(\delta_{\lambda} X\right) \xi_{j}\right) \hat{\xi}_{j}=\sum_{1 \leq j \leq h}(-1)^{j+1}\left\langle\xi_{j} \mid \delta_{\lambda} X\right\rangle \hat{\xi}_{j} \\
& =\sum_{\ell=1}^{\kappa} \sum_{1 \leq j \leq h}(-1)^{j+1} \mu_{\ell} \lambda^{\ell}\left\langle\xi_{j} \mid v_{\ell}\right\rangle \hat{\xi}_{j}=\sum_{i=1}^{\kappa} \sum_{\ell=1}^{\kappa} \sum_{p_{j}=i}(-1)^{j+1} \mu_{\ell} \lambda^{\ell}\left\langle\xi_{j} \mid v_{\ell}\right\rangle \hat{\xi}_{j} \\
& =\sum_{i=1}^{\kappa} \mu_{i} \lambda^{i} \sum_{p_{j}=i}(-1)^{j+1}\left\langle\xi_{j} \mid v_{i}\right\rangle \hat{\xi}_{j}=0,
\end{aligned}
$$

by $(21)$. Then $i\left(\delta_{\lambda} X\right) \xi \in \mathfrak{m}$ that is therefore homogeneous.

Finally, if $\omega=* \xi$, then $\mathfrak{g} \perp \mathfrak{m}$ by [17], Section 1.6.2., p.25. This achieves the proof of the first part of the theorem.

To prove the second part of the theorem, we notice that, by Remark 2.1.3, we can find two bases $\left\{w_{1}, \ldots, w_{n-h}\right\}$ of $\mathfrak{m}$ and $\left\{v_{1}, \ldots, v_{h}\right\}$ of $\mathfrak{h}$ such that all the $w_{j}$ 's and the $v_{j}$ 's have pure weights, i.e. such that

$$
w_{j} \in \mathfrak{m}_{p_{j}}, \text { for } j=1, \ldots, n-h \text { and } v_{j} \in \mathfrak{h}_{q_{j}}, \text { for } j=1, \ldots, h .
$$

Since $\left\{w_{1}, \ldots, w_{n-h}, v_{1}, \ldots, v_{h}\right\}$ is a basis of $\mathfrak{g}$, we can take the dual basis $\left\{\omega_{1}, \ldots, \omega_{n-h}, \xi_{1}, \ldots, \xi_{h}\right\}$ such that

$$
\left\langle\omega_{i} \mid w_{j}\right\rangle=\delta_{i j}, \quad\left\langle\xi_{i} \mid v_{j}\right\rangle=\delta_{i j}, \quad\left\langle\omega_{i} \mid v_{j}\right\rangle=\left\langle\xi_{i} \mid w_{j}\right\rangle=0
$$

Since the vectors $w_{1}, \ldots, w_{n-h}, v_{1}, \ldots, v_{h}$ are linearly independent, we can always assume that $\omega_{1} \wedge \cdots \wedge \omega_{n-h} \wedge \xi_{1} \wedge \cdots \wedge \xi_{h}=d V$. Then we put

$$
\omega:=\omega_{1} \wedge \cdots \wedge \omega_{n-h} \quad \text { and } \quad \xi:=\xi_{1} \wedge \cdots \wedge \xi_{h} .
$$

We have

$$
\mathfrak{m}:=\{X \in \mathfrak{g}: i(X) \xi=0\}, \quad \mathfrak{h}:=\{X \in \mathfrak{g}: i(X) \omega=0\} .
$$

Indeed, if $X=\sum_{\ell} \lambda_{\ell} w_{\ell} \in \mathfrak{m}$, then, again by identity 6 (ii) of [29], p.90,

$$
i(X) \xi=\sum_{1 \leq j \leq h}(-1)^{j+1}\left\langle\xi_{j} \mid X\right\rangle \hat{\xi}_{j}=0,
$$

so that $\mathfrak{m} \subset\{X \in \mathfrak{g}: i(X) \xi=0\}$. On the other hand, $\operatorname{dim} \mathfrak{m}=n-h=\operatorname{dim}\{X \in \mathfrak{g}: i(X) \xi=0\}$, and the first identity in $(22)$ follows. The proof of the second identity is similar. 
To prove that $d_{0} \xi=0$ we recall that, by Proposition 2.2.6, $d_{0} \xi=\beta \wedge \xi$. In turn this is possible only if $d_{0} \xi=0$, otherwise $d_{0} \xi$ and $\xi$ would have the same weight. Analogously we prove that $d_{0} \omega=0$.

We define now a new (equivalent) scalar product $\langle\cdot, \cdot\rangle_{0}$ in $\mathfrak{g}$ making the basis $\left\{w_{1}, \ldots, w_{n-h}, v_{1}\right.$, $\left.\ldots, v_{h}\right\}$ (and therefore also the dual basis $\left\{\omega_{1}, \ldots, \omega_{n-h}, \xi_{1}, \ldots, \xi_{h}\right\}$ ) orthonormal. If we denote by $*_{0}$ the Hodge duality operator associated with the scalar product $\langle\cdot, \cdot\rangle_{0}$ and with $d V$ the volume form, we want to show that $*_{0} \xi=\omega$, i.e. that

$$
\alpha \wedge \xi=\langle\alpha, \omega\rangle_{0} d V \quad \text { for all } \alpha \in \bigwedge^{n-h} \mathfrak{g} .
$$

An orthonormal basis of $\bigwedge^{n-h} \mathfrak{g}$ is given by

$$
\Sigma^{n-h}:=\left\{\omega_{i_{1}} \wedge \cdots \wedge \omega_{i_{\ell}} \wedge \xi_{j_{1}} \wedge \cdots \wedge \xi_{j_{n-h-\ell}}, i_{1}<i_{2}<\cdots<i_{\ell}, j_{1}<j_{2}<\cdots<j_{n-h-\ell}\right\},
$$

where the cases $\ell=0$ and $\ell=n-h$ are allowed. Thus, it is enough to test (23) for $\alpha \in \Sigma^{n-h}$. If now $0 \leq \ell<n-h$ (i.e. if there is at least one factor $\xi_{k}$ ), clearly both terms in (23) are zero. Thus, it is enough to take $\alpha=\omega_{1} \wedge \cdots \wedge \omega_{i_{n-h}}=\omega$, so that

$$
\alpha \wedge \xi=d V \quad \text { and } \quad\langle\alpha, \omega\rangle_{0}=1
$$

yielding (23).

Thus we obtain $d_{0} *_{0} \xi=d_{0} \omega=0$ and $d_{0} *_{0} \omega= \pm d_{0} \xi=0$, achieving the proof of the theorem.

2.2.2. Components along complementary subgroups. Given $\mathbb{M}, \mathbb{H}$, complementary subgroups of $\mathbb{G}$, the elements $m \in \mathbb{M}$ and $h \in \mathbb{H}$ such that $g=m h$ are unique because of $\mathbb{M} \cap \mathbb{H}=\{e\}$ and are denoted as components of $g$ along $\mathbb{M}$ and $\mathbb{H}$ or as projections of $g$ on $\mathbb{M}$ and $\mathbb{H}$.

Proposition 2.2.9. If $\mathbb{M}, \mathbb{H}$ are complementary subgroups in $\mathbb{G}$ there is $c_{0}=c_{0}(\mathbb{M}, \mathbb{H})>0$ such that

$$
c_{0}(\|m\|+\|h\|) \leq\|m h\| \leq\|m\|+\|h\|, \quad \text { for all } m \in \mathbb{M}, h \in \mathbb{H} .
$$

Proof. The right hand side follows by the triangular inequality in Definition 3. To prove the left hand side inequality, let us set

$$
c_{0}:=\inf \{\|m h\|:\|m\|+\|h\|=1\} .
$$

Clearly $c_{0}>0$ : indeed it is a minimum that cannot be zero since $m h=e$ would imply $m=h^{-1}=e$ (since the subgroups are complementary). Then the general statement follows by dilation.

The following Lemma will be useful many times along this paper (see also Lemma 3.9 and Remark 3.10 in [33]).

Lemma 2.2.10. Let $\mathbb{G}$ be a step $\kappa$ group. There is $C=C(\mathbb{G})>0$ such that

$$
\left\|p^{-1} q^{-1} p q\right\| \leq C\left(\|p\|^{\frac{1}{\kappa}}\|q\|^{\frac{\kappa-1}{\kappa}}+\|q\|^{\frac{1}{\kappa}}\|p\|^{\frac{\kappa-1}{\kappa}}\right), \quad \text { for all } p, q \in \mathbb{G},
$$

and consequently

$$
\left\|q^{-1} p q\right\| \leq\|p\|+C\left(\|p\|^{\frac{1}{\kappa}}\|q\|^{\frac{\kappa-1}{\kappa}}+\|q\|^{\frac{1}{\kappa}}\|p\|^{\frac{\kappa-1}{\kappa}}\right), \quad \text { for all } p, q \in \mathbb{G} .
$$

Proof. First we prove (25). By (6), $p^{-1} q^{-1} p q$ is a vector-valued polynomial in the variables $p_{1}, \ldots, p_{n}$ and $q_{1}, \ldots, q_{n}$. We denote by $\left(p^{-1} q^{-1} p q\right)_{\ell}$ the $\ell$ component, homogeneous of degree $\alpha_{\ell}$, for $\ell=1, \ldots, n$. By Remark 2.1.1, $\left(p^{-1} q^{-1} p q\right)_{\ell}$ is a linear combination of homogeneous monomials of the form $p_{h}^{s} q_{k}^{r}$, with $r, s$ non negative integers, $s \alpha_{h}+r \alpha_{k}=\alpha_{\ell}$. But $\left(p^{-1} q^{-1} p q\right)_{\ell}$ vanishes for $p=e$ and for $q=e$, and hence the previous homogeneous $\alpha_{\ell}$-degree polynomials contain only mixed monomials in the variables $p_{1}, \ldots, p_{n}$ and $q_{1}, \ldots, q_{n}$. These monomials have the form $p_{h}^{s} q_{k}^{r}$, with $r, s>0$. Therefore, using (16),

$$
\left|\left(p^{-1} q^{-1} p q\right)_{\ell}\right| \leq C \sum_{i=1}^{\alpha_{\ell}-1}\|p\|^{i}\|q\|^{\alpha_{\ell}-i}
$$


for a given geometric constant $C>0$. Moreover

$$
\|p\|^{i}\|q\|^{\alpha_{\ell}-i} \leq\|p\|\|q\|^{\alpha_{\ell}-1}+\|p\|^{\alpha_{\ell}-1}\|q\|, \quad \text { for all } i=1, \ldots, \alpha_{\ell}-1
$$

since $\|p\|^{i}\|q\|^{\alpha_{\ell}-i}$ is estimated by $\|p\|\|q\|^{\alpha_{\ell}-1}$ when $\|p\| \leq\|q\|$ and by $\|p\|^{\alpha_{\ell}-1}\|q\|$ when $\|p\| \geq\|q\|$. Hence there is a constant $C_{\ell}>0$ such that

$$
\left|\left(p^{-1} q^{-1} p q\right)_{\ell}\right|^{1 / \alpha_{\ell}} \leq C_{\ell}\left(\|p\|^{1 / \alpha_{\ell}}\|q\|^{\left(\alpha_{\ell}-1\right) / \alpha_{\ell}}+\|p\|^{\left(\alpha_{\ell}-1\right) / \alpha_{\ell}}\|q\|^{1 / \alpha_{\ell}}\right) .
$$

Now (25) follows and, in turn, (26) can be derived from the inequality $\left\|q^{-1} p q\right\| \leq\|p\|+\left\|p^{-1} q^{-1} p q\right\|$.

Corollary 2.2.11. Let $\mathbb{M}, \mathbb{H}$ be complementary subgroups of a step $\kappa$ group $\mathbb{G}$. If $m, \bar{m} \in \mathbb{M}$ and $h, \bar{h} \in \mathbb{H}$ are such that

$$
m h=\bar{h} \bar{m},
$$

then,

$$
\begin{gathered}
\|m\| \leq \frac{1}{c_{0}}\|\bar{m}\|+\frac{C}{c_{0}}\left(\|\bar{m}\|^{\frac{1}{\kappa}}\|\bar{h}\|^{\frac{\kappa-1}{\kappa}}+\|\bar{h}\|^{\frac{1}{\kappa}}\|\bar{m}\|^{\frac{\kappa-1}{\kappa}}\right), \\
\|h\| \leq \frac{1}{c_{0}}\|\bar{h}\|+\frac{C}{c_{0}}\left(\|\bar{m}\|^{\frac{1}{\kappa}}\|\bar{h}\|^{\frac{\kappa-1}{\kappa}}+\|\bar{h}\|^{\frac{1}{\kappa}}\|\bar{m}\|^{\frac{\kappa-1}{\kappa}}\right) .
\end{gathered}
$$

Finally, for all $\delta>0$ there is $c(\delta)=c(\delta, \mathbb{M}, \mathbb{H})>0$ such that $\|m h\| \leq \delta$ yields

$$
\|m\| \leq c(\delta)\|\bar{m}\|^{1 / \kappa}, \quad\|h\| \leq c(\delta)\|\bar{h}\|^{1 / \kappa} .
$$

Proof. We denote as $p_{\mathbb{M}} \in \mathbb{M}$ and $p_{\mathbb{H}} \in \mathbb{H}$ the unique 'components' of a generic $p \in \mathbb{G}$ such that

$$
p=p_{\mathbb{M}} p_{\mathbb{H}} .
$$

With this notation,

$$
m h=(\bar{h} \bar{m})_{\mathbb{M}}(\bar{h} \bar{m})_{\mathbb{H}} ;
$$

and, by uniqueness of the components,

$$
m=(\bar{h} \bar{m})_{\mathbb{M}}=\left(\bar{h} \bar{m} \bar{h}^{-1}\right)_{\mathbb{M}}, \quad h=(\bar{h} \bar{m})_{\mathbb{H}}=\left(\bar{m}^{-1} \bar{h} \bar{m}\right)_{\mathbb{H}}
$$

Hence, by (24) and (26),

$$
\|m\|=\left\|\left(\bar{h} \bar{m} \bar{h}^{-1}\right)_{\mathbb{M}}\right\| \leq \frac{1}{c_{0}}\left\|\bar{h} \bar{m} \bar{h}^{-1}\right\| \leq \frac{1}{c_{0}}\|\bar{m}\|+\frac{C}{c_{0}}\left(\|\bar{m}\|^{\frac{1}{\kappa}}\|\bar{h}\|^{\frac{\kappa-1}{\kappa}}+\|\bar{h}\|^{\frac{1}{\kappa}}\|\bar{m}\|^{\frac{\kappa-1}{\kappa}}\right) .
$$

The other inequality is proved in the same way. Finally to prove (27) we use that $\|m h\| \leq \delta$ yields $\|\bar{h}\|,\|\bar{m}\| \leq \delta / c_{0}$.

From now on, we will keep the convention introduced in the proof of Corollary 2.2.11 and we will denote as $g_{\mathbb{M}}$ and $g_{\mathbb{H}}$ the components of $g \in \mathbb{G}$. More precisely our convention is as follows, when $\mathbb{M}, \mathbb{H}$ are complementary subgroups in $\mathbb{G}, \mathbb{M}$ will always be the first 'factor' and $\mathbb{H}$ the second one and $g_{\mathbb{M}} \in \mathbb{M}$ and $g_{\mathbb{H}} \in \mathbb{H}$ are the unique elements such that

$$
g=g_{\mathbb{M}} g_{\mathbb{H}} \cdot
$$

We stress that this notation is ambiguous because each component $g_{\mathbb{M}}$ and $g_{\mathbb{H}}$ depends on both the complementary subgroups $\mathbb{M}$ and $\mathbb{H}$ and also on the order under which they are taken. The projection maps $\mathbf{P}_{\mathbb{M}}: \mathbb{G} \rightarrow \mathbb{M}$ and $\mathbf{P}_{\mathbb{H}}: \mathbb{G} \rightarrow \mathbb{H}$ are defined as

$$
\mathbf{P}_{\mathbb{M}}(g):=g_{\mathbb{M}}, \quad \mathbf{P}_{\mathbb{H}}(g):=g_{\mathbb{H}}
$$

We will collect now a few properties of components and projection maps. In particular, in Proposition 2.2.14 we prove that projection maps $\mathbf{P}_{\mathbb{M}}: \mathbb{G} \rightarrow \mathbb{M}$ and $\mathbf{P}_{\mathbb{H}}: \mathbb{G} \rightarrow \mathbb{H}$ are $C^{\infty}$ (indeed polynomial) as maps from $\mathbb{G}=\mathbb{R}^{n} \rightarrow \mathbb{G}=\mathbb{R}^{n}$. Nevertheless, quite differently from Euclidean spaces, $\mathbf{P}_{\mathbb{M}}$ and $\mathbf{P}_{\mathbb{H}}$, in general, are not even Lipschitz maps, from $\mathbb{G}$ to $\mathbb{M}$ or to $\mathbb{H}$, when $\mathbb{G}, \mathbb{M}$ 
and $\mathbb{H}$ are endowed with the restriction of the natural left invariant distance $d$ of $\mathbb{G}$ (see Example 2.2.15). This fact has many unpleasant consequences. One of them is related with the difficulty of controlling in an easy way the measure, or even the Hausdorff dimension, of the projection of sets (see e.g. [7]).

Observe that in general, $\left(g_{\mathbb{M}}\right)^{-1} \neq\left(g^{-1}\right)_{\mathbb{M}}$ and $\left(g_{\mathbb{H}}\right)^{-1} \neq\left(g^{-1}\right)_{\mathbb{H}}$. We will use the notation

$$
g_{\mathbb{M}}^{-1}=\left(g_{\mathbb{M}}\right)^{-1} \text { and } g_{\mathbb{H}}^{-1}=\left(g_{\mathbb{H}}\right)^{-1} \text {. }
$$

The sizes of the projections $\mathbf{P}_{\mathbb{M}}(p)$ and $\mathbf{P}_{\mathbb{H}}(p)$ control the distance of $p \in \mathbb{G}$ from the complementary subspaces $\mathbb{M}$ and $\mathbb{H}$. The control is different when considering the distance of $p$ from the first component $\mathbb{M}$ or from the second component $\mathbb{H}$ or if the second component is a normal subgroup.

Corollary 2.2.12. Let $\mathbb{M}$, $\mathbb{H}$ be complementary subgroups of a step $\kappa$ group $\mathbb{G}$ and let $\mathbb{G}=\mathbb{M} \mathbb{H}$. Then

$$
c_{0}\left\|\mathbf{P}_{\mathbb{H}}(p)\right\| \leq \operatorname{dist}(p, \mathbb{M}) \leq\left\|\mathbf{P}_{\mathbb{H}}(p)\right\| \quad \text { for all } p \in \mathbb{G},
$$

where $c_{0}$ is the constant in (24). Moreover, if $\mathbb{H}$ is a normal subgroup of $\mathbb{G}$ then

$$
c_{0}\left\|\mathbf{P}_{\mathbb{M}}(p)\right\| \leq \operatorname{dist}(p, \mathbb{H}) \leq\left\|\mathbf{P}_{\mathbb{M}}(p)\right\| \quad \text { for all } p \in \mathbb{G} .
$$

If $\mathbb{H}$ is not a normal subgroup of $\mathbb{G}$, then there is $c_{1}=c_{1}(\mathbb{M}, \mathbb{H})>1$ such that

$$
\frac{1}{c_{1}}\left\|\mathbf{P}_{\mathbb{M}}(p)\right\|^{\kappa} \leq \operatorname{dist}(p, \mathbb{H}) \leq c_{1}\left\|\mathbf{P}_{\mathbb{M}}(p)\right\|^{1 / \kappa} \quad \text { if }\|p\|=1 .
$$

Proof. Using the notation in (28),

$$
\operatorname{dist}(p, \mathbb{M})=\inf \left\{\left\|p^{-1} m\right\|: m \in \mathbb{M}\right\} \leq\left\|p_{\mathbb{H}}^{-1} p_{\mathbb{M}}^{-1} p_{\mathbb{M}}\right\|=\left\|p_{\mathbb{H}}\right\| .
$$

On the other side, for $\varepsilon>0$, let $\bar{m} \in \mathbb{M}$ be such that $\left\|\bar{m}^{-1} p\right\| \leq \operatorname{dist}(p, \mathbb{M})+\varepsilon$ then

$$
c_{0}\left\|p_{\mathbb{H}}\right\| \leq c_{0}\left(\left\|\bar{m}^{-1} p_{\mathbb{M}}\right\|+\left\|p_{\mathbb{H}}\right\|\right) \leq\left\|\bar{m}^{-1} p\right\| \leq \operatorname{dist}(p, \mathbb{M})+\varepsilon .
$$

To estimate the distance of $p$ from $\mathbb{H}$, when $\mathbb{H}$ is normal in $\mathbb{G}$, observe

$$
\begin{aligned}
\operatorname{dist}(p, \mathbb{H}) & =\inf \left\{\left\|h^{-1} p_{\mathbb{M}} p_{\mathbb{H}}\right\|: h \in \mathbb{H}\right\} \\
& =\inf \left\{\left\|p_{\mathbb{M}} p_{\mathbb{M}}^{-1} h^{-1} p_{\mathbb{M}} p_{\mathbb{H}}\right\|: h \in \mathbb{H}\right\} \\
& \leq\left\|p_{\mathbb{M}}\right\|+\inf \left\{\left\|p_{\mathbb{M}}^{-1} h^{-1} p_{\mathbb{M}} p_{\mathbb{H}}\right\|: h \in \mathbb{H}\right\} \\
& =\left\|p_{\mathbb{M}}\right\| \quad \text { with } h=p_{\mathbb{M}} p_{\mathbb{H}} p_{\mathbb{M}}^{-1} .
\end{aligned}
$$

On the other side, given $\varepsilon>0$ let $h \in \mathbb{H}$ be such that dist $(p, \mathbb{H})+\varepsilon>\left\|h^{-1} p\right\|$, then

$$
\operatorname{dist}(p, \mathbb{H})+\varepsilon>\left\|h^{-1} p_{\mathbb{M}} p_{\mathbb{H}}\right\|=\left\|p_{\mathbb{M}} p_{\mathbb{M}}^{-1} h^{-1} p_{\mathbb{M}} p_{\mathbb{H}}\right\| \geq c_{0}\left\|p_{\mathbb{M}}\right\|
$$

by Proposition 2.2.9. This concludes the proof of the second statement. Finally, in the last case, think of $\mathbb{G}$ as $\mathbb{G}=\mathbb{H} \mathbb{M}$ and write $p=\bar{h} \bar{m}$. Then, the estimate of the distance of a point $p$ from the first component of a splitting gives

$$
\tilde{c}_{0}\|\bar{m}\| \leq \operatorname{dist}(p, \mathbb{H}) \leq\|\bar{m}\|,
$$

where $\tilde{c}_{0}$ is the constant in Proposition 2.2 .9 , but related to the splitting $\mathbb{G}=\mathbb{H} \mathbb{M}$. Now the inequalities in (27) give the thesis.

Observe that (29) cannot be improved without additional assumptions. Indeed

Example 2.2.13. Let $\mathbb{G}$ be the Heisenberg group $\mathbb{H}^{1}=\left(\mathbb{R}^{3}, \cdot\right)$ with the group law

$$
x \cdot y=\left(x_{1}, x_{2}, x_{3}\right) \cdot\left(y_{1}, y_{2}, y_{3}\right):=\left(x_{1}+y_{1}, x_{2}+y_{2}, x_{3}+y_{3}+\left(x_{1} y_{2}-x_{2} y_{1}\right) / 2 .\right.
$$

Let

$$
\mathbb{V}=\left\{x=\left(x_{1}, 0,0\right): x_{1} \in \mathbb{R}\right\} \text { and } \underset{15}{\mathbb{W}}=\left\{x=\left(0, x_{2}, x_{3}\right): x_{2}, x_{3} \in \mathbb{R}\right\}
$$


$\mathbb{V}$ and $\mathbb{W}$ are complementary subgroups in $\mathbb{H}^{1}, \mathbb{W}$ is a normal subgroup while $\mathbb{V}$ is not a normal subgroup.

For $s \in(0,1)$ let $p_{s}:=(1, s,-s / 2) \in \mathbb{H}^{1}$. Then as $s \rightarrow 0$,

$$
\begin{aligned}
\operatorname{dist}\left(p_{s}, \mathbb{V}\right) & =\inf \left\{\left\|v^{-1} p_{s}\right\|: v \in \mathbb{V}\right\} \\
& =\inf \left\{\left\|\left(1-x_{1}, s,-s\left(x_{1}+1\right) / 2\right)\right\|: x_{1} \in \mathbb{R}\right\} \approx \sqrt{s} ; \\
\left\|\mathbf{P}_{\mathbb{W}}\left(p_{s}\right)\right\| & =\|(0, s, 0)\| \approx s .
\end{aligned}
$$

On the other side, for $s \in(0,1)$ let $q_{s}:=(1, s, s / 2) \in \mathbb{H}^{1}$. Then, as $s \rightarrow 0$,

$$
\begin{aligned}
\operatorname{dist}\left(q_{s}, \mathbb{V}\right) & =\inf \left\{\left\|v^{-1} q_{s}\right\|: v \in \mathbb{V}\right\} \\
& =\inf \left\{\left\|\left(1-x_{1}, s, s\left(1-x_{1}\right) / 2\right)\right\|: x_{1} \in \mathbb{R}\right\} \approx s \\
\left\|\mathbf{P}_{\mathbb{W}}\left(q_{s}\right)\right\| & =\|(0, s, s)\| \approx \sqrt{s} .
\end{aligned}
$$

See also Example 2.2.15.

Proposition 2.2.14. Let $\mathbb{M}, \mathbb{H}$ be complementary subgroups of $\mathbb{G}$, then the projection maps $\mathbf{P}_{\mathbb{M}}$ : $\mathbb{G} \rightarrow \mathbb{M}$ and $\mathbf{P}_{\mathbb{H}}: \mathbb{G} \rightarrow \mathbb{H}$ defined in (28) are polynomial maps. More precisely, if $\kappa$ is the step of $\mathbb{G}$, there are $2 \kappa$ matrices $A^{1}, \ldots, A^{\kappa}, B^{1}, \ldots, B^{\kappa}$, depending on $\mathbb{M}$ and $\mathbb{H}$, such that

$$
A^{j} \text { and } B^{j} \text { are }\left(n_{j}, n_{j}\right) \text {-matrices }
$$

and, with the notations of (3),

$$
\begin{aligned}
& \mathbf{P}_{\mathbb{M}} g=\left(A^{1} g^{1}, A^{2}\left(g^{2}-\mathcal{Q}^{2}\left(A^{1} g^{1}, B^{1} g^{1}\right)\right), \ldots, A^{\kappa}\left(g^{\kappa}-\mathcal{Q}^{\kappa}\left(A^{1} g^{1}, \ldots, B^{\kappa-1} g^{\kappa-1}\right)\right)\right) ; \\
& \mathbf{P}_{\mathbb{H}} g=\left(B^{1} g^{1}, B^{2}\left(g^{2}-\mathcal{Q}^{2}\left(A^{1} g^{1}, B^{1} g^{1}\right)\right), \ldots, B^{\kappa}\left(g^{\kappa}-\mathcal{Q}^{\kappa}\left(A^{1} g^{1}, \ldots, B^{\kappa-1} g^{\kappa-1}\right)\right)\right) ;
\end{aligned}
$$

$A^{j}$ is the identity on $\mathbb{M}^{j}$, and $B^{j}$ is the identity on $\mathbb{H}^{j}$.

Proof. Recall the notation

$$
g=\left(g^{1}, \ldots, g^{\kappa}\right), \quad \mathbf{P}_{\mathbb{M}}(g)=g_{\mathbb{M}}=\left(g_{\mathbb{M}}^{1}, \ldots, g_{\mathbb{M}}^{\kappa}\right), \quad \mathbf{P}_{\mathbb{H}}(g)=g_{\mathbb{H}}=\left(g_{\mathbb{H}}^{1}, \ldots, g_{\mathbb{H}}^{\kappa}\right) .
$$

Let $d$ and $n-d$ be respectively the linear dimensions of $\mathbb{M}$ and $\mathbb{H}$. Because $\mathbb{M}$ and $\mathbb{H}$ are complementary subgroups there are a $(n-d, n)$-matrix $M$, a $(d, n)$-matrix $H$, such that $\mathbb{M}=\{x \in \mathbb{G}: M x=0\}$ and $\mathbb{H}=\{x \in \mathbb{G}: H x=0\}$ and such the $(n, n)$-matrix $\left[\begin{array}{c}M \\ H\end{array}\right]$ is non singular. In particular,

$$
M g_{\mathbb{M}}=0 \text { and } H g_{\mathbb{H}}=0 \text {, for all } g \in \mathbb{G} \text {. }
$$

Notice that both $M$ and $H$ have the form

$$
M=\left[\begin{array}{cccc}
M^{1} & 0 & \cdots & 0 \\
0 & M^{2} & & \vdots \\
\vdots & & \ddots & 0 \\
0 & \cdots & 0 & M^{\kappa}
\end{array}\right], \quad H=\left[\begin{array}{cccc}
H^{1} & 0 & \cdots & 0 \\
0 & H^{2} & & \vdots \\
\vdots & & \ddots & 0 \\
0 & \cdots & 0 & H^{\kappa}
\end{array}\right],
$$

where, for $1 \leq j \leq \kappa, M^{j}$ is an $\left(n_{j}-d_{j}, n_{j}\right)$ matrix, $H^{j}$ is an $\left(d_{j}, n_{j}\right)$ matrix and the $\left(n_{j}, n_{j}\right)$ matrix $\left[\begin{array}{l}M^{j} \\ H^{j}\end{array}\right]$ is non singular. Here we have denoted as $d_{j}$ and $n_{j}-d_{j}$ the dimensions of the corresponding layers of $\mathbb{M}$ and $\mathbb{H}$. 
By definition, $g_{\mathbb{M}}$ and $g_{\mathbb{H}}$ are the solutions of the system of $2 n$ equations in the $2 n$ unknowns $g_{\mathbb{M}, 1}, \ldots, g_{\mathbb{M}, n}$ and $g_{\mathbb{H}, 1}, \ldots, g_{\mathbb{H}, n}$

$$
\begin{aligned}
& g_{\mathbb{M}}^{1}+g_{\mathbb{H}}^{1}=g^{1}, \\
& g_{\mathbb{M}}^{2}+g_{\mathbb{H}}^{2}+\mathcal{Q}^{2}\left(g_{\mathbb{M}}^{1}, g_{\mathbb{H}}^{1}\right)=g^{2}, \\
& \quad \vdots \\
& g_{\mathbb{M}}^{\kappa}+g_{\mathbb{H}}^{\kappa}+\mathcal{Q}^{\kappa}\left(g_{\mathbb{M}}^{1}, \ldots, g_{\mathbb{M}}^{\kappa-1}, g_{\mathbb{H}}^{1}, \ldots, g_{\mathbb{H}}^{\kappa-1}\right)=g^{\kappa}, \\
& M g_{\mathbb{M}}=0, \\
& H g_{\mathbb{H}}=0 .
\end{aligned}
$$

This system can be solved layer by layer. From what stated before, we know that the linear system of $2 n_{1}$ equations and unknowns

$$
\begin{aligned}
& g_{\mathbb{M}}^{1}+g_{\mathbb{H}}^{1}=g^{1}, \\
& M^{1} g_{\mathbb{M}}^{1}=0, \\
& H^{1} g_{\mathbb{H}}^{1}=0,
\end{aligned}
$$

has a unique solution $g_{\mathbb{M}}^{1}, g_{\mathbb{H}}^{1}$ depending linearly on the components of $g^{1}$. We denote

$$
g_{\mathbb{M}}^{1}=A^{1} g^{1} \text { and } g_{\mathbb{H}}^{1}=B^{1} g^{1} .
$$

Then we find the unique solution of the linear system in the $2 n_{2}$ unknowns $g_{\mathbb{M}}^{2}$ and $g_{\mathbb{H}}^{2}$

$$
\begin{aligned}
& g_{\mathbb{M}}^{2}+g_{\mathbb{H}}^{2}=g^{2}-\mathcal{Q}^{2}\left(g_{\mathbb{M}}^{1}, g_{\mathbb{H}}^{1}\right), \\
& M^{2} g_{\mathbb{M}}^{2}=0 \\
& H^{2} g_{\mathbb{H}}^{2}=0
\end{aligned}
$$

and we denote as $A^{2}$ and $B^{2}$ the matrices such that

$$
\begin{aligned}
g_{\mathbb{M}}^{2} & =A^{2}\left(g^{2}-\mathcal{Q}^{2}\left(g_{\mathbb{M}}^{1}, g_{\mathbb{H}}^{1}\right)\right)=A^{2}\left(g^{2}-\mathcal{Q}^{2}\left(A^{1} g^{1}, B^{1} g^{1}\right)\right), \\
g_{\mathbb{H}}^{1} & =B^{2}\left(g^{2}-\mathcal{Q}^{2}\left(g_{\mathbb{M}}^{1}, g_{\mathbb{H}}^{1}\right)\right)=B^{2}\left(g^{2}-\mathcal{Q}^{2}\left(A^{1} g^{1}, B^{1} g^{1}\right)\right) .
\end{aligned}
$$

Then we iterate the procedure up to the layer $\kappa$.

In order to prove (iv), observe that if $g \in \mathbb{M}$ then $\mathbf{P}_{\mathbb{M}}(g)=g$ and $\mathbf{P}_{\mathbb{H}}(g)=0$ hence

$$
\begin{aligned}
& g=\left(A^{1} g^{1}, A^{2}\left(g^{2}-\mathcal{Q}^{2}\left(A^{1} g^{1}, B^{1} g^{1}\right)\right), \ldots, A^{\kappa}\left(g^{\kappa}-\mathcal{Q}^{\kappa}\left(A^{1} g^{1}, \ldots, B^{\kappa-1} g^{\kappa-1}\right)\right)\right), \\
& 0=\left(B^{1} g^{1}, B^{2}\left(g^{2}-\mathcal{Q}^{2}\left(A^{1} g^{1}, B^{1} g^{1}\right)\right), \ldots, B^{\kappa}\left(g^{\kappa}-\mathcal{Q}^{\kappa}\left(A^{1} g^{1}, \ldots, B^{\kappa-1} g^{\kappa-1}\right)\right)\right) .
\end{aligned}
$$

From these we get $g^{1}=A^{1} g^{1}$ and $0=B^{1} g^{1}$, for all $g \in \mathbb{M}$. Looking at the second layer, notice that $0=B^{1} g^{1}$ yields $\left.\mathcal{Q}^{2}\left(A^{1} g^{1}, B^{1} g^{1}\right)\right)=0$, hence we have $g^{2}=A^{2}\left(g^{2}\right)$ and $0=B^{2} g^{2}$, for all $g \in \mathbb{M}$. The procedure can be repeated up to the layer $\kappa$.

As we anticipated, $\mathbf{P}_{\mathbb{M}}$ and $\mathbf{P}_{\mathbb{H}}$ are not, in general, Lipschitz maps when $\mathbb{G}, \mathbb{M}$ and $\mathbb{H}$ are endowed with the restriction of the distance $d$ of $\mathbb{G}$.

Example 2.2.15. Let $\mathbb{G}$ be the Heisenberg group $\mathbb{H}^{1}=\left(\mathbb{R}^{3}, \cdot\right)$ and let $\mathbb{V}$ and $\mathbb{W}$ be the subgroups defined in Example 2.2.13.

When we consider $\mathbb{H}^{1}=\mathbb{V} \cdot \mathbb{W}$ the projections $\mathbf{P}_{\mathbb{V}}$ and $\mathbf{P}_{\mathbb{W}}$ are

$$
\mathbf{P}_{\mathbb{V}}\left(x_{1}, x_{2}, x_{3}\right)=\left(x_{1}, 0,0\right), \quad \mathbf{P}_{\mathbb{W}}\left(x_{1}, x_{2}, x_{3}\right)=\left(0, x_{2}, x_{3}-x_{1} x_{2} / 2\right) .
$$

Here $\mathbf{P}_{\mathbb{W}}: \mathbb{H}^{1} \rightarrow \mathbb{W}$ is not Lipschitz. Indeed, let $q=(1,1,0)$ and $p_{\varepsilon}=(1+\varepsilon, 1+\varepsilon, 0)$, then $\mathbf{P}_{\mathbb{W}} q=(0,1,-1 / 2)$ and $\mathbf{P}_{\mathbb{W}} p_{\varepsilon}=\left(0,1+\varepsilon,-(1+\varepsilon)^{2} / 2\right)$. Hence, as $\varepsilon \rightarrow 0^{+}$,

$$
\left\|q^{-1} p\right\|=\|(\varepsilon, \varepsilon, 0)\| \approx \varepsilon, \quad\left\|\left(\mathbf{P}_{\mathbb{W}} q\right)^{-1} \mathbf{P}_{\mathbb{W}} p_{\varepsilon}\right\|=\left\|\left(0, \varepsilon,-\varepsilon-\varepsilon^{2} / 2\right)\right\| \approx \sqrt{\varepsilon}
$$


When we consider $\mathbb{H}^{1}=\mathbb{W} \cdot \mathbb{V}$, then $\mathbf{P}_{\mathbb{V}}$ and $\mathbf{P}_{\mathbb{W}}$ are

$$
\mathbf{P}_{\mathbb{V}}\left(x_{1}, x_{2}, x_{3}\right)=\left(x_{1}, 0,0\right), \quad \mathbf{P}_{\mathbb{W}}\left(x_{1}, x_{2}, x_{3}\right)=\left(0, x_{2}, x_{3}+x_{1} x_{2} / 2\right)
$$

Let $q=(1,0,0)$ and $p_{\varepsilon}=(1, \varepsilon, \varepsilon / 2)$, then $\mathbf{P}_{\mathbb{W}} q=(0,0,0)$ and $\mathbf{P}_{\mathbb{W}} p_{\varepsilon}=(0, \varepsilon, \varepsilon)$. Hence

$$
\left\|q^{-1} p\right\|=\|(0, \varepsilon, 0)\| \approx \varepsilon, \quad\left\|\left(\mathbf{P}_{\mathbb{W}} q\right)^{-1} \mathbf{P}_{\mathbb{W}} p_{\varepsilon}\right\|=\|(0, \varepsilon, \varepsilon)\| \approx \sqrt{\varepsilon}
$$

as $\varepsilon \rightarrow 0^{+}$. In this case too, $\mathbf{P}_{\mathbb{W}}$ is not a Lipschitz map.

The example shows that both the projections either on the first factor or on the second factor can be non Lipschitz. Notice that in both cases we were considering projections on the normal factor. Indeed the projection on the complement of a normal subgroup is always metric Lipschitz continuous.

Proposition 2.2.16. Let $\mathbb{M}, \mathbb{H}$ be complementary subgroups of $\mathbb{G}$. Then

$$
\text { if } \mathbb{H} \text { is a normal subgroup then } \mathbf{P}_{\mathbb{M}} \text { is Lipschitz; }
$$$$
\text { if } \mathbb{M} \text { is a normal subgroup then } \mathbf{P}_{\mathbb{H}} \text { is Lipschitz. }
$$

Proof. (i) For all $g=m h$ and $\bar{g}=\bar{m} \bar{h}$, we have

$$
\mathbf{P}_{\mathbb{M}}\left(g^{-1} \bar{g}\right)=\mathbf{P}_{\mathbb{M}}\left(h^{-1} m^{-1} \bar{m} \bar{h}\right)=\mathbf{P}_{\mathbb{M}}\left(m^{-1} \bar{m} \bar{m}^{-1} m h^{-1} m^{-1} \bar{m} \bar{h}\right)=m^{-1} \bar{m} .
$$

Hence, for all $g, \bar{g} \in \mathbb{G}, \mathbf{P}_{\mathbb{M}}\left(g^{-1} \bar{g}\right)=\left(\mathbf{P}_{\mathbb{M}} g\right)^{-1} \mathbf{P}_{\mathbb{M}} \bar{g}$ and, by (24),

$$
\left\|\left(\mathbf{P}_{\mathbb{M}} g\right)^{-1} \mathbf{P}_{\mathbb{M}} \bar{g}\right\| \leq\left(\left\|\mathbf{P}_{\mathbb{M}}\left(g^{-1} \bar{g}\right)\right\|+\left\|\mathbf{P}_{\mathbb{H}}\left(g^{-1} \bar{g}\right)\right\|\right) \leq c_{0}^{-1}\left\|g^{-1} \bar{g}\right\| .
$$

(ii) As before, $\mathbf{P}_{\mathbb{H}}\left(g^{-1} \bar{g}\right)=\left(\mathbf{P}_{\mathbb{H}} g\right)^{-1} \mathbf{P}_{\mathbb{H}} \bar{g}$ and eventually,

$$
\left\|\left(\mathbf{P}_{\mathbb{H}} g\right)^{-1} \mathbf{P}_{\mathbb{H}} \bar{g}\right\| \leq c_{0}^{-1}\left\|g^{-1} \bar{g}\right\|, \quad \text { for all } g, \bar{g} \in \mathbb{G} \text {. }
$$

Even if the projections are not Lipschitz we have the following control on the measure of projected sets.

Lemma 2.2.17. Let $\mathbb{M}$, $\mathbb{H}$ be complementary subgroups of $\mathbb{G}$. Denote by $d_{t} \leq d_{m}$ respectively, the topological and the metric dimensions of $\mathbb{M}$. Then there is $c=c(\mathbb{M}, \mathbb{H})>0$ such that,

$$
\mathcal{L}^{d_{t}}\left(\mathbf{P}_{\mathbb{M}}(B(p, r))\right)=c r^{d_{m}},
$$

for all balls $B(p, r) \subset \mathbb{G}$.

Proof. Define

Observe that $c(\mathbb{M}, \mathbb{H})>0$.

$$
c=c(\mathbb{M}, \mathbb{H}):=\mathcal{L}^{d_{t}}\left(\mathbf{P}_{\mathbb{M}}(B(e, 1))\right) .
$$

Indeed the Lebesgue measure $\mathcal{L}^{d_{t}}$ is non-zero on $\mathbb{M}$ being the image under the exponential map of the Lebesgue measure on the $d_{t}$-dimensional Lie algebra of $\mathbb{M}$. Moreover

$$
\mathbb{M}=\bigcup_{i \in \mathbb{N}} \mathbf{P}_{\mathbb{M}}(B(e, i)) .
$$

Hence there exists $i \in \mathbb{N}$ such that $\mathcal{L}^{d_{t}}\left(\mathbf{P}_{\mathbb{M}}(B(e, i))\right)>0$. By group dilations $\mathbf{P}_{\mathbb{M}}(B(e, r))=$ $\mathbf{P}_{\mathbb{M}}\left(\delta_{r} B(e, 1)\right)=\delta_{r} \mathbf{P}_{\mathbb{M}}(B(e, 1))$, for all $r>0$. Therefore $\mathcal{L}^{d_{t}}\left(\mathbf{P}_{\mathbb{M}}(B(e, 1))\right)>0$ and

$$
\mathcal{L}^{d_{t}}\left(\mathbf{P}_{\mathbb{M}}(B(e, r))\right)=c r^{d_{m}} .
$$

To prove that also $\mathcal{L}^{d_{t}}\left(\mathbf{P}_{\mathbb{M}}(B(p, r))\right)=\mathcal{L}^{d_{t}}\left(\mathbf{P}_{\mathbb{M}}(p \cdot B(e, r))\right)=c r^{d_{m}}$ we prove that, for any fixed $p \in \mathbb{G}$, the map $\Phi_{p}: \mathbb{M} \rightarrow \mathbb{M}$ defined as $\Phi_{p}(m):=\mathbf{P}_{\mathbb{M}}(p \cdot m)$, has unit Jacobian determinant. That is, we prove that, for any measurable $\mathcal{E} \subset \mathbb{G}$,

$$
\mathcal{L}^{d_{t}}\left(\mathbf{P}_{\mathbb{M}}(p \cdot \mathcal{E})\right)=\mathcal{L}^{d_{t}}\left(\mathbf{P}_{\mathbb{M}}(\mathcal{E})\right) .
$$


With the notations in Lemma 2.2.14,

$$
\begin{gathered}
\Phi_{p}(m)=\mathbf{P}_{\mathbb{M}}\left(p^{1}+m^{1}, p^{2}+m^{2}+\mathcal{Q}^{2}\left(p^{1}, m^{1}\right), \ldots, p^{\kappa}+m^{\kappa}+\mathcal{Q}^{\kappa}\left(p^{1}, \ldots, m^{\kappa-1}\right)\right) \\
=\left(A^{1}\left(p^{1}+m^{1}\right), A^{2}\left(p^{2}+m^{2}+\mathcal{Q}^{2}\left(p^{1}, m^{1}\right)-\mathcal{Q}^{2}\left(A^{1}\left(p^{1}+m^{1}\right), B^{1}\left(p^{1}+m^{1}\right)\right), \ldots\right.\right. \\
\left.\ldots, A^{\kappa}\left(p^{\kappa}+m^{\kappa}+\text { function of }\left(m^{1}, \ldots, m^{\kappa-1}\right)\right)\right) .
\end{gathered}
$$

Hence the Jacobian of $\Phi_{p}$ has the form

$$
\left[\frac{\partial \Phi_{p}}{\partial m}\right]=\left[\begin{array}{cccc}
A^{1} & 0 & \cdots & 0 \\
* & A^{2} & & \vdots \\
\vdots & & \ddots & 0 \\
* & \cdots & * & A^{\kappa}
\end{array}\right]
$$

and $\operatorname{det}\left[\frac{\partial \Phi_{p}}{\partial m}\right]=1$ because each $A^{j}$ is the identity on $\mathbb{M}^{j}$.

\subsubsection{Graphs.}

Definition 8. Let $\mathbb{H}$ be a homogeneous subgroup of $\mathbb{G}$. We say that a set $S \subset \mathbb{G}$ is a (left) $\mathbb{H}$-graph (or a left graph in direction $\mathbb{H}$ ) if $S$ intersects each left coset of $\mathbb{H}$ at most in one point.

When $\mathbb{H}$ admits a complementary subgroup $\mathbb{M}$, then

$$
S \text { is a } \mathbb{H} \text {-graph if and only if } S=\operatorname{graph}(\varphi) \text {, }
$$

i.e. if

$$
S=\{\xi \cdot \varphi(\xi): \xi \in \mathcal{E}\}
$$

for $\varphi: \mathcal{E} \subset \mathbb{M} \rightarrow \mathbb{H}$. By uniqueness of the components along $\mathbb{M}$ and $\mathbb{H}$, if $S=\operatorname{graph}(\varphi)$ then $\varphi$ is uniquely determined among all functions from $\mathbb{M}$ to $\mathbb{H}$.

More generally, $\mathbb{H}$ graphs are graphs of functions (in the above sense) even if complementary subgroups of $\mathbb{H}$ fail to exist. Indeed, if $A \subset \mathbb{G}$ intersects each left coset of $\mathbb{H}$ exactly in one point, and $S$ is a $\mathbb{H}$-graph, then there is a unique function $\varphi: \mathcal{E} \subset A \rightarrow \mathbb{H}$ such that $S$ is the graph of $\varphi$, that is

$$
S=\operatorname{graph}(\varphi):=\{\xi \cdot \varphi(\xi): \xi \in \mathcal{E}\} .
$$

Conversely, for any $\psi: \mathcal{D} \subset A \rightarrow \mathbb{H}$ the set graph $(\psi)$ is an $\mathbb{H}$-graph.

From now on we will consider mainly graphs of functions acting between complementary subgroups. Nevertheless it is relevant to mention that examples of $\mathbb{H}$-graphs that are not graphs of functions acting between complementary subgroups have been considered inside the Heisenberg groups $\mathbb{H}^{n}=\mathbb{R}^{2 n+1}$. These sets are given as

$$
S=\left\{\left(x_{1}, \cdots, y_{n}, \varphi\left(x_{1}, \cdots, y_{n}\right)\right)\right\} \subset \mathbb{H}^{n} .
$$

In our notation such an $S$ is a $\mathbb{T}$-graph, where $\mathbb{T}$ is the center of $\mathbb{H}^{n}$. The left cosets of $\mathbb{T}$ are parametrized over the set $A=H \mathbb{H}_{e}^{n}$. We recall that the center $\mathbb{T}$ has no complementary subgroup in $\mathbb{H}^{n}$, and in general there is not a couple of complementary subgroups $\mathbb{M}, \mathbb{H}$ of $\mathbb{H}^{n}$ and a $\psi: \mathbb{M} \rightarrow \mathbb{H}$ such that $S=\operatorname{graph}(\psi)$, even locally.

If a set $S \subset \mathbb{G}$ is an intrinsic graph then it keeps being an intrinsic graph after left translations or group dilations.

Proposition 2.2.18. Let $\mathbb{H}$ be a homogeneous subgroup of $\mathbb{G}$. If $S$ is a $\mathbb{H}$-graph then, for all $\lambda>0$ and for all $q \in \mathbb{G}, \delta_{\lambda} S$ and $q \cdot S$ are $\mathbb{H}$-graphs. 
If $\mathbb{M}, \mathbb{H}$ are complementary subgroups in $\mathbb{G}$, if $S=\operatorname{graph}(\varphi)$ with $\varphi: \mathcal{E} \subset \mathbb{M} \rightarrow \mathbb{H}$, then

$$
\begin{aligned}
& \text { For all } \lambda>0, \delta_{\lambda} S=\operatorname{graph}\left(\varphi_{\lambda}\right) \text {, with } \\
& \varphi_{\lambda}: \delta_{\lambda} \mathcal{E} \subset \mathbb{M} \rightarrow \mathbb{H} \text { and } \\
& \varphi_{\lambda}(m)=\delta_{\lambda} \varphi\left(\delta_{1 / \lambda} m\right), \text { for } m \in \delta_{\lambda} \mathcal{E} .
\end{aligned}
$$

For any $q \in \mathbb{G}, \quad q \cdot S=\operatorname{graph}\left(\varphi_{q}\right)$, where

$$
\begin{aligned}
& \varphi_{q}: \mathcal{E}_{q} \subset \mathbb{M} \rightarrow \mathbb{H}, \quad \mathcal{E}_{q}=\left\{m: \mathbf{P}_{\mathbb{M}}\left(q^{-1} m\right) \in \mathcal{E}\right\} \text { and } \\
& \varphi_{q}(m)=\left(\mathbf{P}_{\mathbb{H}}\left(q^{-1} m\right)\right)^{-1} \cdot \varphi\left(\mathbf{P}_{\mathbb{M}}\left(q^{-1} m\right)\right), \text { for all } m \in \mathcal{E}_{q} .
\end{aligned}
$$

Proof. If $x, x^{\prime} \in S$ and $x \neq x^{\prime}$ then, by definition of $\mathbb{H}$-graph, $x, x^{\prime}$ belong to different left cosets of $\mathbb{H}$. Then $\delta_{\lambda} x, \delta_{\lambda} x^{\prime}$ belong to different cosets of $\mathbb{H}$, because $\mathbb{H}$ is a homogeneous subgroup, and also $q \cdot x, q \cdot x^{\prime}$ belong to different cosets of $\mathbb{H}$, by elementary properties of cosets (see e.g. [30, chapter 2 , section 4]). By definition, these facts prove that both $\delta_{\lambda} S$ and $q \cdot S$ are $\mathbb{H}$-graphs and that there are $\varphi_{\lambda}$ and $\varphi_{q}$ such that $\delta_{\lambda} S=\operatorname{graph}\left(\varphi_{\lambda}\right)$ and $q \cdot S=\operatorname{graph}\left(\varphi_{q}\right)$.

To prove (30) observe that, by uniqueness of the components, $\delta_{\lambda}(m \cdot \varphi(m))=m^{\prime} \cdot \varphi\left(m^{\prime}\right)$ implies that $\delta_{\lambda} m=m^{\prime}$ and that $\varphi_{\lambda}=\delta_{\lambda} \circ \varphi \circ \delta_{1 / \lambda}$.

To prove (31) observe that, because $p_{\mathbb{H}}^{-1}=p^{-1} \cdot p_{\mathbb{M}}$, for all $p \in \mathbb{G}$, then $\left(q^{-1} \cdot m\right)_{\mathbb{H}}^{-1}=m^{-1} \cdot q$. $\left(q^{-1} \cdot m\right)_{\mathbb{M}}$, hence

$$
\begin{aligned}
\operatorname{graph}\left(\varphi_{q}\right) & =\left\{m \cdot \varphi_{q}(m): m \in \mathcal{E}_{q}\right\} \\
& =\left\{m \cdot\left(q^{-1} \cdot m\right)_{\mathbb{H}}^{-1} \cdot \varphi\left(\left(q^{-1} \cdot m\right)_{\mathbb{M}}\right): m \in \mathcal{E}_{q}\right\} \\
& =\left\{m \cdot m^{-1} \cdot q \cdot\left(q^{-1} \cdot m\right)_{\mathbb{M}} \cdot \varphi\left(\left(q^{-1} \cdot m\right)_{\mathbb{M}}\right):\left(q^{-1} \cdot m\right)_{\mathbb{M}} \in \mathcal{E}\right\} \\
& =q \cdot \operatorname{graph}(\varphi) .
\end{aligned}
$$

Remark 2.2.19. From (31) and the continuity of the projection maps $\mathbf{P}_{\mathbb{M}}$ and $\mathbf{P}_{\mathbb{H}}$ it follows that the continuity of a function is preserved by translations. Precisely, given $q=q_{\mathbb{M}} q_{\mathbb{H}}$ and $f: \mathbb{M} \rightarrow \mathbb{H}$, then the translated function $f_{q}$ is continuous in $m \in \mathbb{M}$ if and only if the function $f$ is continuous in the corresponding point $\left(q^{-1} m\right)_{\mathbb{M}}$.

Remark 2.2.20. The algebraic expression of $\varphi_{q}$ in Proposition 2.2.18 can be made more explicit when $\mathbb{G}$ is a semi-direct product of $\mathbb{M}, \mathbb{H}$. Precisely

If $\mathbb{M}$ is normal in $\mathbb{G}$ then $\varphi_{q}(m)=q_{\mathbb{H}} \varphi\left(\left(q^{-1} m\right)_{\mathbb{M}}\right), \quad$ for $m \in \mathcal{E}_{q}=q \mathcal{E}\left(q_{\mathbb{H}}\right)^{-1}$.

If $\mathbb{H}$ is normal in $\mathbb{G}$ then $\varphi_{q}(m)=\left(q^{-1} m\right)_{\mathbb{H}}^{-1} \varphi\left(q_{\mathbb{M}}^{-1} m\right)$, for $m \in \mathcal{E}_{q}=q_{\mathbb{M}} \mathcal{E}$.

If both $\mathbb{M}$ and $\mathbb{H}$ are normal in $\mathbb{G}$ we get the well known formula

$$
\varphi_{q}(m)=q_{\mathbb{H}} \varphi\left(q_{\mathbb{M}}^{-1} m\right), \quad \text { for } m \in \mathcal{E}_{q}=q_{\mathbb{M}} \mathcal{E} .
$$

See also [5, Proposition 3.6].

\section{INTRINSIC LIPSCHITZ FUNCTIONS}

3.1. General definitions. Intrinsic Lipschitz functions in $\mathbb{G}$ are functions, acting between complementary subgroups of $\mathbb{G}$, with graphs non intersecting naturally defined cones. Hence, the notion of intrinsic Lipschitz graph respects the geometry of the ambient group $\mathbb{G}$. Precisely a $\mathbb{H}$-graph $S$ is said to be an intrinsic Lipschitz $\mathbb{H}$-graph if $S$ intersects intrinsic cones with axis $\mathbb{H}$, fixed opening and vertex on $S$ only in the vertex. Intrinsic Lipschitz functions appeared for the first time in [21] and were studied, more diffusely, in $[25,26]$.

We begin with two definitions of intrinsic (closed) cones. The first one, Definition 9, is more general because it does not require that $\mathbb{H}$ is a complemented subgroup. 
Definition 9. Let $\mathbb{H}$ be a homogeneous subgroup of $\mathbb{G}, q \in \mathbb{G}$. The cones $X(q, \mathbb{H}, \alpha)$ with axis $\mathbb{H}$, vertex $q$, opening $\alpha, 0 \leq \alpha \leq 1$ are

$$
X(q, \mathbb{H}, \alpha):=q \cdot X(e, \mathbb{H}, \alpha) \text {, where } X(e, \mathbb{H}, \alpha):=\{p: \operatorname{dist}(p, \mathbb{H}) \leq \alpha\|p\|\} .
$$

When working with functions acting between complementary subgroups, we will consider also the following family of cones.

Definition 10. If $\mathbb{M}, \mathbb{H}$ are complementary subgroups in $\mathbb{G}, q \in \mathbb{G}$ and $\beta \geq 0$, the cones $C_{\mathbb{M}, \mathbb{H}}(q, \beta)$, with base $\mathbb{M}$, axis $\mathbb{H}$, vertex $q$, opening $\beta$ are defined as

$$
C_{\mathbb{M}, \mathbb{H}}(q, \beta)=q \cdot C_{\mathbb{M}, \mathbb{H}}(e, \beta) \text {, where } C_{\mathbb{M}, \mathbb{H}}(e, \beta)=\left\{p:\left\|p_{\mathbb{M}}\right\| \leq \beta\left\|p_{\mathbb{H}}\right\|\right\} \text {. }
$$

Observe that

$$
\mathbb{H}=X(e, \mathbb{H}, 0)=C_{\mathbb{M}, \mathbb{H}}(e, 0), \quad \mathbb{G}=X(e, \mathbb{H}, 1)=\overline{\cup_{\beta>0} C_{\mathbb{M}, \mathbb{H}}(e, \beta)} .
$$

Moreover, the cones $C_{\mathbb{M}, \mathbb{H}}(q, \beta)$ are 'equivalent' with the cones $X(q, \mathbb{H}, \alpha)$. Indeed

Proposition 3.1.1. If $\mathbb{M}, \mathbb{H}$ are complementary subgroups in $\mathbb{G}$ then, for any $\alpha \in(0,1)$ there is $\beta \geq 1$, depending on $\alpha, \mathbb{M}$ and $\mathbb{H}$, such that

$$
C_{\mathbb{M}, \mathbb{H}}(q, 1 / \beta) \subset X(q, \mathbb{H}, \alpha) \subset C_{\mathbb{M}, \mathbb{H}}(q, \beta),
$$

Proof. It is enough to prove the claim with $q=e$. Let us prove the first inequality. By definition and by dilation invariance of the cones, it is enough to prove that for each $\alpha \in(0,1)$ there is $\beta>0$ such that

$$
\left\|p_{\mathbb{M}}\right\| \leq \frac{1}{\beta}\left\|p_{\mathbb{H}}\right\| \Longrightarrow \operatorname{dist}(p, \mathbb{H}) \leq \alpha \quad \text { for all } p \in \mathbb{G} \text { with }\|p\|=1 .
$$

From $\left\|p_{\mathbb{M}}\right\| \leq \frac{1}{\beta}\left\|p_{\mathbb{H}}\right\|$ and $(24)$

$$
\left(1+\frac{1}{\beta}\right)\left\|p_{\mathbb{M}}\right\| \leq \frac{1}{\beta}\left(\left\|p_{\mathbb{M}}\right\|+\left\|p_{\mathbb{H}}\right\|\right) \leq \frac{1}{c_{0} \beta} .
$$

Then, from (29) and (33), there is $c_{1}=c_{1}(\mathbb{M}, \mathbb{H})>0$ such that

$$
\operatorname{dist}(p, \mathbb{H}) \leq c_{1}\left\|p_{\mathbb{M}}\right\|^{1 / \kappa} \leq \frac{c_{1}}{\left(c_{0}(1+\beta)\right)^{1 / \kappa}} \leq \alpha
$$

if $\beta$ is large enough, for all $p \in \mathbb{G}$ with $\|p\|=1$. This completes the proof of (32).

Let us prove the second inequality in the claim of the theorem. Once more, by dilation invariance of the cones, it is enough to prove that for each $\beta>0$ there is $\alpha \in(0,1)$ such that

$$
\operatorname{dist}(p, \mathbb{H}) \leq \alpha \Longrightarrow\left\|p_{\mathbb{M}}\right\| \leq \beta\left\|p_{\mathbb{H}}\right\| \quad \text { for all } p \in \mathbb{G} \text { with }\|p\|=1 .
$$

From (29) we have

$$
\left\|p_{\mathbb{M}}\right\| \leq c_{1}^{1 / \kappa} \operatorname{dist}(p, \mathbb{H})^{1 / \kappa} \leq c_{1}^{1 / \kappa} \alpha^{1 / \kappa} .
$$

Hence, from $\|p\|=1$ and $(24)$,

$$
1-c_{1}^{1 / \kappa} \alpha^{1 / \kappa} \leq 1-\left\|p_{\mathbb{M}}\right\| \leq\left\|p_{\mathbb{H}}\right\|
$$

Finally

$$
\left\|p_{\mathbb{M}}\right\| \leq \frac{c_{1}^{1 / \kappa} \alpha^{1 / \kappa}}{1-c_{1}^{1 / \kappa} \alpha^{1 / \kappa}}\left\|p_{\mathbb{H}}\right\|
$$

and we can choose $\alpha$ so small that the fraction is less than $\beta$.

Now we introduce the basic definition of this paragraph. 
Definition 11. Let $\mathbb{H}$ be an homogeneous subgroup, not necessarily complemented in $\mathbb{G}$. We say that an $\mathbb{H}$-graph $S$ is an intrinsic Lipschitz $\mathbb{H}$-graph if there is $\alpha \in(0,1)$ such that,

$$
S \cap X(p, \mathbb{H}, \alpha)=\{p\}, \quad \text { for all } p \in S .
$$

If $\mathbb{M}, \mathbb{H}$ are complementary subgroups in $\mathbb{G}$, we say that $f: \mathcal{E} \subset \mathbb{M} \rightarrow \mathbb{H}$ is intrinsic Lipschitz in $\mathcal{E}$ when graph $(f)$ is an intrinsic Lipschitz $\mathbb{H}$-graph.

We say that $f: \mathcal{E} \subset \mathbb{M} \rightarrow \mathbb{H}$ is intrinsic L-Lipschitz in $\mathcal{E}$ for $L \geq 0$ if for all $\tilde{L}>L$

$$
C_{\mathbb{M}, \mathbb{H}}(p, 1 / \tilde{L}) \cap \operatorname{graph}(f)=\{p\} \quad \text { for all } p \in \operatorname{graph}(f) .
$$

The Lipschitz constant of $f$ in $\mathcal{E}$ is the infimum of all $L>0$ such that $f$ is $L$-Lipschitz.

It follows from Proposition 3.1.1 that $f$ is intrinsic Lipschitz in $\mathcal{E}$ if and only if it is intrinsic $L$-Lipschitz for an appropriate constant $L$, depending on $\alpha, f$ and $\mathbb{M}$.

Because of Proposition 2.2.18 and Definition 10 left translations of intrinsic Lipschitz $\mathbb{H}$-graphs, or of intrinsic L-Lipschitz functions, will be intrinsic Lipschitz $\mathbb{H}$-graphs, or intrinsic L-Lipschitz functions. We state this in the following theorem.

Theorem 3.1.2. If $\mathbb{G}$ is a Carnot group, then for all $q \in \mathbb{G}$,

if $S \subset \mathbb{G}$ is an intrinsic Lipschitz $\mathbb{H}$-graph then $q \cdot S$ is an intrinsic Lipschitz $\mathbb{H}$-graph;

if $f: \mathcal{E} \subset \mathbb{M} \rightarrow \mathbb{H}$ is intrinsic L-Lipschitz then $f_{q}: \mathcal{E}_{q} \subset \mathbb{M} \rightarrow \mathbb{H}$ is intrinsic L-Lipschitz.

The geometric definition of intrinsic Lipschitz graphs, proposed in Definition 11, has equivalent algebraic forms (see also [5], [24], [26]).

Proposition 3.1.3. Let $\mathbb{M}, \mathbb{H}$ be complementary subgroups in $\mathbb{G}, f: \mathcal{E} \subset \mathbb{M} \rightarrow \mathbb{H}$ and $L>0$. Then (i) to (iii) are equivalent.

(i) $\quad f$ is intrinsic L-Lipschitz in $\mathcal{E}$.

(ii) $\quad\left\|\mathbf{P}_{\mathbb{H}}\left(\bar{q}^{-1} q\right)\right\| \leq L\left\|\mathbf{P}_{\mathbb{M}}\left(\bar{q}^{-1} q\right)\right\| \quad$ for all $q, \bar{q} \in \operatorname{graph}(f)$.

(iii) $\quad\left\|f_{\bar{q}^{-1}}(m)\right\| \leq L\|m\| \quad$ for all $\bar{q} \in \operatorname{graph}(f)$ and $m \in \mathcal{E}_{\bar{q}^{-1}}$.

Proof. The equivalence between (i) and (ii) follows from definition (10), observing that if $\bar{q} \in$ $\operatorname{graph}(f)$, then $C_{\mathbb{M}, \mathbb{H}}(\bar{q}, 1 / L) \cap \operatorname{graph}(f)=\{\bar{q}\}$ is equivalent with $C_{\mathbb{M}, \mathbb{H}}(e, 1 / L) \cap \operatorname{graph}\left(f_{\bar{q}^{-1}}\right)=\{e\}$. The equivalence of (ii) and (iii) follows once more from the definition of cone and left invariance of the definition. Indeed we recall that $f_{\bar{q}^{-1}}(m)=\left((f(\bar{m}) m)_{\mathbb{H}}\right)^{-1} f\left(\bar{m}(f(\bar{m}) m)_{\mathbb{M}}\right)$, then $(\bar{q} m)_{\mathbb{H}}=$ $(\bar{m} f(\bar{m}) m)_{\mathbb{H}}=(f(\bar{m}) m)_{\mathbb{H}}$ and $(\bar{q} m)_{\mathbb{M}}=(\bar{m} f(\bar{m}) m)_{\mathbb{M}}=\bar{m}(f(\bar{m}) m)_{\mathbb{M}}$.

Remark 3.1.4. $f$ is intrinsic Lipschitz if and only if the distance of two points $q, \bar{q} \in \operatorname{graph}(f)$ is bounded by the norm of the projection of $\bar{q}^{-1} q$ on the domain $\mathbb{M}$. Precisely, $f: \mathcal{E} \subset \mathbb{M} \rightarrow \mathbb{H}$ is intrinsic Lipschitz if and only if there is a constant $C>0$ such that

$$
\left\|\bar{q}^{-1} q\right\| \leq C\left\|\mathbf{P}_{\mathbb{M}}\left(\bar{q}^{-1} q\right)\right\|, \quad \text { for all } q, \bar{q} \in \operatorname{graph}(f) .
$$

The relations between the constant $C$ and the Lipschitz constant $L$ of $f$ follow from (24): if $f$ is intrinsic $L$-Lipschitz then

$$
\left\|\bar{q}^{-1} q\right\| \leq(1+L)\left\|\mathbf{P}_{\mathbb{M}}\left(\bar{q}^{-1} q\right)\right\|, \quad \text { for all } q, \bar{q} \in \operatorname{graph}(f) ;
$$

conversely, if $\left\|\bar{q}^{-1} q\right\| \leq c_{0}(1+L)\left\|\mathbf{P}_{\mathbb{M}}\left(\bar{q}^{-1} q\right)\right\|$ then

$$
\left\|\mathbf{P}_{\mathbb{H}}\left(\bar{q}^{-1} q\right)\right\| \leq L\left\|\mathbf{P}_{\mathbb{M}}\left(\bar{q}^{-1} q\right)\right\|, \quad \text { for all } q, \bar{q} \in \operatorname{graph}(f)
$$

and $f$ is intrinsic $L$-Lipschitz. 
Remark 3.1.5. If $\mathbb{G}$ is the semi-direct product of $\mathbb{M}$ and $\mathbb{H}$, (ii) and (iii) of Proposition 3.1.3 take a more explicit form. Indeed, recalling Remark 2.2.20, we get

(i) If $\mathbb{M}$ is normal in $\mathbb{G}$ then $f$ is intrinsic L-Lipschitz if and only if

$$
\left\|f(\bar{m})^{-1} f(m)\right\| \leq L\left\|f(\bar{m})^{-1} \bar{m}^{-1} m f(\bar{m})\right\| \quad \text { for all } m, \bar{m} \in \mathcal{E} .
$$

(ii) If $\mathbb{H}$ is normal in $\mathbb{G}$ then

$$
\left((\bar{q} m)^{-1}\right)_{\mathbb{H}} f\left((\bar{q} m)_{\mathbb{M}}\right)=m^{-1} f(\bar{m})^{-1} m f(\bar{m} m),
$$

hence (iii) of Proposition 3.1.3 becomes

$$
\left\|m^{-1} f(\bar{m})^{-1} m f(\bar{m} m)\right\| \leq L\|m\| \quad \text { for all } m, \bar{m} \in \mathcal{E} .
$$

(iii) If $\mathbb{G}$ is a direct product of $\mathbb{M}$ and $\mathbb{H}$ we get the well known expression for Lipschitz functions

$$
\left\|f(\bar{m})^{-1} f(m)\right\| \leq L\left\|\bar{m}^{-1} m\right\|, \quad \text { for all } m, \bar{m} \in \mathcal{E} .
$$

In this case intrinsic Lipschitz functions are the same as the usual metric Lipschitz functions from $\left(\mathbb{M}, d_{\infty}\right)$ to $\left(\mathbb{H}, d_{\infty}\right)$.

Remark 3.1.6. It is a natural question to ask if intrinsic Lipschitz functions are metric Lipschitz functions provided that appropriate choices of the metrics in the domain or in the target spaces are made. The answer is almost always negative. Nevertheless something relevant can be stated. Given $f: \mathcal{E} \subset \mathbb{M} \rightarrow \mathbb{H}$, we consider the function $d_{\mathbb{M}, f}=d_{f}: \mathcal{E} \times \mathcal{E} \rightarrow \mathbb{R}^{+}$defined as

$$
d_{f}\left(m_{1}, m_{2}\right):=\frac{1}{2}\left(\left\|\left(q_{1}^{-1} q_{2}\right)_{\mathbb{M}}\right\|+\left\|\left(q_{2}^{-1} q_{1}\right)_{\mathbb{M}}\right\|\right) \quad \text { for all } m_{1}, m_{2} \in \mathcal{E}
$$

where $q_{i}:=m_{i} \cdot f\left(m_{i}\right) \in \operatorname{graph}(f)$.

If $f$ is an intrinsic $L$-Lipschitz function then $d_{f}$ is a quasi distance in $\mathcal{E}$. By quasi distance we mean that $d_{f}$ is a distance with the weaker triangular inequality (35). Moreover the parametric function $\Phi_{f}$

$$
\Phi_{f}:\left(\mathcal{E}, d_{f}\right) \rightarrow\left(\mathbb{G}, d_{\infty}\right), \quad \Phi_{f}(m):=m \cdot f(m), \quad \text { for all } m \in \mathcal{E} .
$$

is a metric Lipschitz function. That is, $\Phi_{f}$ is a metric Lipschitz parametrization of graph $(f)$ provided we endow $\mathcal{E}$ with the quasi distance $d_{f}$. We stress that in general it is impossible to find a unique quasi distance working for all the intrinsic Lipschitz functions. Notice that this is possible exactly when $\mathbb{H}$ is a normal subgroup.

Let us check that $d_{f}$ is a quasi distance. Clearly $d_{f}$ is symmetric and $m_{1}=m_{2}$ yields $d_{f}\left(m_{1}, m_{2}\right)=$ 0 . About triangular inequality, observe that, for all $q_{1}, q_{2}, q_{3} \in \operatorname{graph}(f)$,

$$
\begin{aligned}
c_{0}\left\|\left(q_{1}^{-1} q_{2}\right)_{\mathbb{M}}\right\| \leq\left\|q_{1}^{-1} q_{2}\right\| & \leq\left\|q_{1}^{-1} q_{3}\right\|+\left\|q_{3}^{-1} q_{2}\right\| \\
& \leq\left\|\left(q_{1}^{-1} q_{3}\right)_{\mathbb{M}}\right\|+\left\|\left(q_{1}^{-1} q_{3}\right)_{\mathbb{H}}\right\|+\left\|\left(q_{3}^{-1} q_{2}\right)_{\mathbb{M}}\right\|+\left\|\left(q_{3}^{-1} q_{2}\right)_{\mathbb{H}}\right\|
\end{aligned}
$$

using here that $f$ is intrinsic Lipschitz to bound the $\mathbb{H}$ components,

$$
\leq(1+L)\left\|\left(q_{1}^{-1} q_{3}\right)_{\mathbb{M}}\right\|+(1+L)\left\|\left(q_{3}^{-1} q_{2}\right)_{\mathbb{M}}\right\| .
$$

Eventually we get the weaker triangular inequality

$$
d_{f}\left(m_{1}, m_{2}\right) \leq\left((1+L) / c_{0}\right)\left(d_{f}\left(m_{1}, m_{3}\right)+d_{f}\left(m_{3}, m_{2}\right)\right) \quad \text { for all } m_{1}, m_{2}, m_{3} \in \mathcal{E} .
$$

Finally, from the preceding computations, we have also that

$$
\left\|q_{1}^{-1} q_{2}\right\| \equiv\left\|\Phi_{f}\left(m_{1}\right)^{-1} \Phi_{f}\left(m_{2}\right)\right\| \leq(1+L) d_{f}\left(m_{1}, m_{2}\right) \quad \text { for all } m_{1}, m_{2} \in \mathcal{E} .
$$

This completes the proof that $\Phi_{f}$ is a Lipschitz parametrization of graph $(f)$.

As anticipated, we have a remarkable special case when $\mathbb{H}$ is a normal subgroup. Indeed, when $\mathbb{H}$ is a normal subgroup,

$$
\left(q_{1}^{-1} q_{2}\right)_{\mathbb{M}}=\left(f\left(m_{1}\right)^{-1} m_{1}^{-1} m_{2} f\left(m_{2}\right)\right)_{\mathbb{M}}=m_{1}^{-1} m_{2}
$$


hence, the distance $d_{f}$ is independent from $f$, precisely

$$
d_{f}\left(m_{1}, m_{2}\right)=\left\|m_{1}^{-1} m_{2}\right\|=d_{\infty}\left(m_{1}, m_{2}\right) .
$$

and $\Phi_{f}:\left(\mathbb{M}, d_{\infty}\right) \rightarrow\left(\mathbb{G}, d_{\infty}\right)$ is a Lipschitz parametrization of graph $(f)$.

Inversely, if $\left\|\Phi_{f}(\bar{m})^{-1} \Phi_{f}(m)\right\| \leq K\left\|\bar{m}^{-1} m\right\|$ then

$$
c_{0}\left\|\left(\Phi_{f}(\bar{m})^{-1} \Phi_{f}(m)\right)_{\mathbb{H}}\right\| \leq K\left\|\bar{m}^{-1} m\right\|=K\left\|\left(\Phi_{f}(\bar{m})^{-1} \Phi_{f}(m)\right)_{\mathbb{M}}\right\| ;
$$

that is $f$ is intrinsic Lipschitz by (ii) of Proposition 3.1.3. It is worth to state this fact as an independent Proposition.

Proposition 3.1.7. Let $\mathbb{M}, \mathbb{H}$ be complementary subgroups of $\mathbb{G}$. Assume that $\mathbb{H}$ is a normal subgroup in $\mathbb{G}$. Then $f: \mathcal{E} \subset \mathbb{M} \rightarrow \mathbb{H}$ is intrinsic Lipschitz in $\mathcal{E}$, if and only if $\Phi_{f}:\left(\mathcal{E}, d_{\infty}\right) \rightarrow$ $\left(\mathbb{G}, d_{\infty}\right)$ is metric Lipschitz, that is if and only if there is $K>1$ such that

$$
\left\|\Phi_{f}(\bar{m})^{-1} \Phi_{f}(m)\right\| \leq K\left\|\bar{m}^{-1} m\right\|, \quad \text { for all } \bar{m}, m \in \mathcal{E} .
$$

If $\mathbb{H}$ is not a normal subgroup Proposition 3.1.7 can be false: even if $f$ is very regular, the 'natural' parametrization of graph $(f)$ given by $\Phi_{f}$ may be non metric Lipschitz. Consider the following example in $\mathbb{H}^{1} \equiv \mathbb{R}^{3}$.

Let $\mathbb{W}, \mathbb{V}$ be the complementary subgroups $\mathbb{V}=\left\{v=\left(v_{1}, 0,0\right)\right\}$ and $\mathbb{W}=\left\{w=\left(0, w_{2}, w_{3}\right)\right\}$ and $f: \mathbb{W} \rightarrow \mathbb{V}$ be the constant map defined by $f(w)=(1,0,0) \in \mathbb{V}$. Then graph $(f)$ is a vertical plane in $\mathbb{R}^{3}$, parallel to $\mathbb{W}$. The 'natural' parametrization $\Phi_{f}: \mathbb{W} \rightarrow \operatorname{graph}(f) \subset \mathbb{H}^{1}$ acts as

$$
\Phi_{f}(w)=\left(1, w_{2}, w_{3}+w_{2} / 2\right) .
$$

Then $\Phi_{f}(e)=(1,0,0)$ and, if $\bar{w}=(0, \varepsilon, 0), \Phi_{f}(\bar{w})=(1, \varepsilon, \varepsilon / 2)$. Hence $\left\|\Phi_{f}(e)^{-1} \cdot \Phi_{f}(\bar{w})\right\|$ is comparable with $\varepsilon^{1 / 2}$ while $\|\bar{w}\|$ is comparable with $\varepsilon$ and (37) fails.

When $\mathbb{M}$ is a normal subgroup then not only $\Phi_{f}$ but $f$ itself is a metric Lipschitz function from $\left(\mathbb{M}, d_{f}\right)$ to $\left(\mathbb{H}, d_{\infty}\right)$. Indeed, in this case,

$$
\left(q_{1}^{-1} q_{2}\right)_{\mathbb{M}}=f\left(m_{1}\right)^{-1} m_{1}^{-1} m_{2} f\left(m_{1}\right) \text { and }\left(q_{1}^{-1} q_{2}\right)_{\mathbb{H}}=f\left(m_{1}\right)^{-1} f\left(m_{2}\right) .
$$

Hence, using (ii) of Proposition 3.1.3, we have

$$
\left\|f\left(m_{1}\right)^{-1} f\left(m_{2}\right)\right\|=\left\|\left(q_{1}^{-1} q_{2}\right)_{\mathbb{H}}\right\| \leq L\left\|\left(q_{1}^{-1} q_{2}\right)_{\mathbb{M}}\right\|=L\left\|f\left(m_{1}\right)^{-1} m_{1}^{-1} m_{2} f\left(m_{1}\right)\right\|,
$$

for all $m_{1}, m_{2} \in \mathcal{E}$. Hence, we get the metric Lipschitz continuity of $f$, that is

$$
\left\|f\left(m_{1}\right)^{-1} f\left(m_{2}\right)\right\| \leq L d_{f}\left(m_{1}, m_{2}\right), \quad \text { for all } m_{1}, m_{2} \in \mathcal{E} .
$$

Finally we notice that it is an open problem to understand if and when metric Lipschitz parameterizations of graph $(f)$, different from the 'natural' parametrization $\Phi_{f}$, exist. This problem was addressed in [15] where the authors proved that, if $S$ is a codimension 1 surface in $\mathbb{H}^{n}$ and if it is somehow more regular than just Lipschitz, then a metric Lipschitz parametrization of $S$ exists (with a parameter space independent of $S$ ). On the contrary, D.Vittone in [8] proves that bi-Lipschitz parameterizations may not exist.

Intrinsic Lipschitz functions, even if non metric Lipschitz, nevertheless are Hölder continuos.

Proposition 3.1.8. Let $\mathbb{M}, \mathbb{H}$ be complementary subgroups in a step $\kappa$ group $\mathbb{G}$. Let $L>0$ and $f: \mathcal{E} \subset \mathbb{M} \rightarrow \mathbb{H}$ be an intrinsic L-Lipschitz function. Then

(i) for all $R>0$ there is $C_{1}=C_{1}(\mathbb{M}, \mathbb{H}, f, R)>0$ such that

$$
\|f(m)\| \leq C_{1} \quad \text { for } m \in \mathcal{E} \text { such that }\|m\| \leq R .
$$

(ii) For all $R>0$, there is $C_{2}=C_{2}\left(\mathbb{G}, \mathbb{M}, \mathbb{H}, C_{1}, L, R\right)>0$ such that

$$
\left\|f(\bar{m})^{-1} f(m)\right\| \leq C_{2}\left\|\bar{m}^{-1} m\right\|^{1 / \kappa} \quad \text { for } m, \bar{m} \in \mathcal{E} \text { with }\|m\|,\|\bar{m}\| \leq R .
$$


Proof. Fix $\bar{m} \in \mathcal{E}$ with $\|\bar{m}\| \leq R$. From (ii) of Proposition 3.1.3 with $q=m f(m)$ and $\bar{q}=\bar{m} f(\bar{m})$, we have

$$
\left\|\left(f(\bar{m})^{-1} \bar{m}^{-1} m\right)_{\mathbb{H}} f(m)\right\| \leq L\left\|\left(f(\bar{m})^{-1} \bar{m}^{-1} m\right)_{\mathbb{M}}\right\|, \quad \text { for all } m \in \mathcal{E} .
$$

Now (38) follows from (39) using (24), triangle inequality and the limitations on $\|m\|$ and on $\|\bar{m}\|$.

To obtain the Hölder estimate observe that, from Corollary 2.2.11 and (38), there is $C_{3}=$ $C_{3}\left(\mathbb{M}, \mathbb{H}, C_{1}, R\right)>0$ such that

$$
\left\|\left(f(\bar{m})^{-1} \bar{m}^{-1} m\right)_{\mathbb{M}}\right\| \leq C_{3}\left\|\bar{m}^{-1} m\right\|^{1 / \kappa}, \quad \text { for all } \bar{m}, m \in \mathcal{E},\|m\|,\|\bar{m}\| \leq R .
$$

Then, from (39),

$$
\left\|\left(f(\bar{m})^{-1} \bar{m}^{-1} m\right)_{\mathbb{H}} f(m)\right\| \leq L C_{3}\left\|\bar{m}^{-1} m\right\|^{1 / \kappa}, \quad \text { for all } \bar{m}, m \in \mathcal{E},\|m\|,\|\bar{m}\| \leq R .
$$

From Lemma 2.2.10 and (38) we have

$$
\left\|f(\bar{m})^{-1} m^{-1} \bar{m} f(\bar{m})\right\| \leq C_{4}\left\|\bar{m}^{-1} m\right\|^{1 / \kappa} ;
$$

from (24), (40) and (41)

$$
\begin{aligned}
\left\|f(\bar{m})^{-1} \bar{m}^{-1} m f(m)\right\| & \leq\left\|\left(f(\bar{m})^{-1} \bar{m}^{-1} m f(m)\right)_{\mathbb{M}}\right\|+\left\|\left(f(\bar{m})^{-1} \bar{m}^{-1} m f(m)\right)_{\mathbb{H}}\right\| \\
& =\left\|\left(f(\bar{m})^{-1} \bar{m}^{-1} m\right)_{\mathbb{M}}\right\|+\left\|\left(f(\bar{m})^{-1} \bar{m}^{-1} m\right)_{\mathbb{H}} f(m)\right\| \\
& \leq(1+L) C_{3}\left\|\bar{m}^{-1} m\right\|^{1 / \kappa}
\end{aligned}
$$

for all $\bar{m}, m \in \mathcal{E}$ with $\|m\|,\|\bar{m}\| \leq R$. Finally, from the last one and from (42)

$$
\left\|f(\bar{m})^{-1} f(m)\right\| \leq\left\|f(\bar{m})^{-1} m^{-1} \bar{m} f(\bar{m})\right\|+\left\|f(\bar{m})^{-1} \bar{m}^{-1} m f(m)\right\| \leq C_{2}\left\|\bar{m}^{-1} m\right\|^{1 / \kappa},
$$

for all $\bar{m}, m \in \mathcal{E}$ with $\|m\|,\|\bar{m}\| \leq R$.

3.2. Surface measure of Lipschitz graphs. Let $\mathbb{M}, \mathbb{H}$ be complementary subgroups in $\mathbb{G}$, let $\mathcal{E} \subset \mathbb{M}$ be an open set. If $f: \mathcal{E} \rightarrow \mathbb{H}$ is intrinsic Lipschitz, then the metric dimension of graph $(f)$ is the same as the metric dimension of the domain $\mathcal{E}$. In fact, we prove below a stronger statement: a Lipschitz graph parametrized on a homogeneous subgroup of dimension $d_{m}$ is (locally) Alhfors $d_{m}$-regular.

A non trivial corollary of this estimate is that 1-codimensional intrinsic Lipschitz graphs are boundaries of sets of locally finite $\mathbb{G}$-perimeter (see [19], Theorem 4.2.9).

We point out that in Euclidean spaces it is always true that the Hausdorff dimension of the graph of a Lipschitz function equals the Hausdorff dimension of its domain, even if the domain fails to be open. Notice that in Euclidean spaces intrinsic Lipschitz functions are the same as Lipschitz functions. In Carnot groups this stronger statement (stronger in the sense that holds also for lower-dimensional domains $\mathcal{E}$ ) is false in general as the following easy example shows: in the Heisenberg group $\mathbb{H}^{1}:=\left(\mathbb{R}^{3}, \cdot\right)$ let $\mathbb{V}$ and $\mathbb{W}$ be the complementary homogeneous subgroups defined as $\mathbb{V}:=\left\{v=\left(v_{1}, 0,0\right): v_{1} \in \mathbb{R}\right\}$ and $\mathbb{W}:=\left\{w=\left(0, w_{2}, w_{3}\right): w_{2}, w_{3} \in \mathbb{R}\right\}$. Let $f: \mathbb{W} \rightarrow \mathbb{V}$ be the intrinsic Lipschitz function (constant) defined as $w=\left(0, w_{2}, w_{3}\right) \mapsto f(w):=(1,0,0)$. Let $\mathcal{E}:=\left\{\left(0, w_{2}, 0\right): w_{2} \in \mathbb{R}\right\}$. Since $\mathcal{E}$ is an horizontal curve, its metric dimension equals 1. But $f(\mathcal{E})=\left\{\left(1, w_{2},-w_{2} / 2\right): w_{2} \in \mathbb{R}\right\}$ is not anymore an horizontal curve, hence its metric dimension is larger than 1 , indeed it equals 2 .

Notice that the proofs of upper and lower bounds for the Hausdorff measure of a Lipschitz graph work trivially in Euclidean spaces. Indeed if $f: \mathbb{R}^{k} \rightarrow \mathbb{R}^{n-k}$ is Lipschitz then the map $\Phi_{f}: \mathbb{R}^{k} \rightarrow \mathbb{R}^{n}$ defined as $\Phi_{f}(x):=(x, f(x))$ is a Lipschitz parametrization of the Euclidean graph of $f$; this gives the upper bound. On the other side, the projection $\mathbb{R}^{n} \equiv \mathbb{R}^{k} \times \mathbb{R}^{n-k} \rightarrow \mathbb{R}^{k}$ is Lipschitz continuous, with Lipschitz constant 1, yielding the lower bound. Such a proof cannot work here. From one side the projection $\mathbf{P}_{\mathbb{M}}$ or $\mathbf{P}_{\mathbb{H}}$ are not Lipschitz continuous, on the other side, as observed in Remark 
3.1.6, the 'natural' parametrization $\Phi_{f}: \mathbb{M} \rightarrow \mathbb{G}, \Phi_{f}(m):=m f(m)$, is almost never a Lipschitz continuous map between the two metric spaces $\mathbb{M}$ and $\mathbb{G}$.

Theorem 3.2.1. Let $\mathbb{M}$, $\mathbb{H}$ be complementary subgroups in $\mathbb{G}$. Let $d_{m}$ denote the metric dimension of $\mathbb{M}$. If $f: \mathbb{M} \rightarrow \mathbb{H}$ is intrinsic L-Lipschitz in $\mathbb{M}$ then there is $c=c(\mathbb{M}, \mathbb{H})>0$ such that,

$$
\left(\frac{c_{0}}{1+L}\right)^{d_{m}} R^{d_{m}} \leq \mathcal{S}_{d}^{d_{m}}(\operatorname{graph}(f) \cap B(p, R)) \leq c(1+L)^{d_{m}} R^{d_{m}}
$$

for all $p \in \operatorname{graph}(f)$ and $R>0$, where $c_{0}$ is the structural constant in Proposition 2.2.9. In particular, graph $(f)$ has metric dimension $d_{m}$.

Proof. The lower bound for $\mathcal{S}_{d}^{d_{m}}(\operatorname{graph}(f))$ is a consequence of Lemma 2.2.17. Indeed, assume $\mathcal{S}_{d}^{d_{m}}(\operatorname{graph}(f) \cap B(p, R))<\infty$. Fix $\varepsilon>0$, choose $r=r(\varepsilon)>0$ and a covering of $\operatorname{graph}(f) \cap B(p, R)$ with closed balls $B_{i}=B\left(p_{i}, r_{i}\right)$ such that $r_{i} \leq r$ and

$$
\sum_{i} r_{i}^{d_{m}} \leq \mathcal{S}_{d}^{d_{m}}(\operatorname{graph}(f) \cap B(p, R))+\varepsilon
$$

Now observe that if $f: \mathbb{M} \rightarrow \mathbb{H}$ is intrinsic $L$-Lipschitz and $p \in \operatorname{graph}(f)$ then

$$
\mathbf{P}_{\mathbb{M}}\left(B\left(p, \frac{c_{0} R}{1+L}\right)\right) \subset \mathbf{P}_{\mathbb{M}}(\operatorname{graph}(f) \cap B(p, R)) \text { for all } R>0 \text {. }
$$

Indeed, $m \in \mathbf{P}_{\mathbb{M}}\left(B\left(p, \frac{c_{0} R}{1+L}\right)\right)$ if and only if there is $h \in \mathbb{H}$ such that $\left\|p^{-1} m h\right\| \leq \frac{c_{0} R}{1+L}$. Hence, if $m \in \mathbf{P}_{\mathbb{M}}\left(B\left(p, \frac{c_{0} R}{1+L}\right)\right)$ then

$$
c_{0}\left\|\mathbf{P}_{\mathbb{M}}\left(p^{-1} m\right)\right\|=c_{0}\left\|\mathbf{P}_{\mathbb{M}}\left(p^{-1} m h\right)\right\| \leq\left\|p^{-1} m h\right\| \leq \frac{c_{0} R}{1+L} .
$$

On the other side, using also the last inequality in Remark 3.1.4,

$$
\begin{aligned}
\left\|p^{-1} m f(m)\right\| & \leq(1+L)\left\|\mathbf{P}_{\mathbb{M}}\left(p^{-1} m f(m)\right)\right\| \\
& =(1+L)\left\|\mathbf{P}_{\mathbb{M}}\left(p^{-1} m\right)\right\| \\
& \leq R .
\end{aligned}
$$

Hence $m f(m) \in \operatorname{graph}(f) \cap B(p, R)$ and $m \in \mathbf{P}_{\mathbb{M}}(\operatorname{graph}(f) \cap B(p, R))$ and (44) is proved.

Denoting as $d_{t} \leq d_{m}$ the topological dimension of $\mathbb{M}$, from (44) and Lemma 2.2.17 we conclude that for all $\varepsilon>0$,

$$
\begin{aligned}
c\left(\frac{c_{0}}{1+L}\right)^{d_{m}} R^{d_{m}} & =\mathcal{L}^{d_{t}}\left(\mathbf{P}_{\mathbb{M}}\left(B\left(p, \frac{c_{0} R}{1+L}\right)\right)\right) \\
& \leq \mathcal{L}^{d_{t}}\left(\mathbf{P}_{\mathbb{M}}(\operatorname{graph}(f) \cap B(p, R))\right) \\
& \leq \sum_{i} \mathcal{L}^{d_{t}}\left(\mathbf{P}_{\mathbb{M}}\left(B_{i}\right)\right) \\
& =c \sum_{i} r_{i}^{d_{m}} \\
& \leq c \mathcal{S}_{d}^{d_{m}}(\operatorname{graph}(f) \cap B(p, R))+\varepsilon,
\end{aligned}
$$

and the left hand side of (43) follows by letting $\varepsilon \rightarrow 0$.

To prove the upper bound in (43), we show that there is $c=c(\mathbb{G}, \mathbb{M}, \mathbb{H}, R, L)>0$ such that for any $p \in \operatorname{graph}(f), R>0$ and $\varepsilon>0$, it is possible to cover graph $(f) \cap B(p, R)$ with less than

$$
N=c \varepsilon^{-d_{m}}
$$


metric balls of radius not exceeding $5 \varepsilon$. Without loss of generality we assume $p=e$ and we fix $\varepsilon$, $0<\varepsilon<1$. By a Vitali covering argument we choose a family of metric balls $B\left(q_{i}, 5 \varepsilon\right)$, such that

$$
\operatorname{graph}(f) \cap B(e, R) \subset \bigcup_{i=1}^{N} B\left(q_{i}, 5 \varepsilon\right) \quad q_{i}=m_{i} f\left(m_{i}\right) \in \operatorname{graph}(f), \quad i=1, \ldots, N,
$$

and the concentric smaller balls $B_{i}:=B\left(q_{i}, \varepsilon\right)$ are pairwise disjoint. We have to estimate the number $N$. With this purpose, recall the semi metric $d_{f}$ defined in Remark 3.1.6 and observe that from $(36)$

$$
2 \varepsilon \leq\left\|q_{i}^{-1} q_{j}\right\| \leq(1+L) d_{f}\left(m_{i}, m_{j}\right) \quad \text { for all } i \neq j .
$$

Hence

$$
\frac{2 \varepsilon}{(1+L)} \leq d_{f}\left(m_{i}, m_{j}\right) \quad \text { for all } m_{i} \neq m_{j}
$$

Denote

$$
\mathcal{E}_{i}:=\left\{m \in \mathbb{M}: d_{f}\left(m, m_{i}\right)<\frac{c_{0} \varepsilon}{(1+L)^{2}}\right\} .
$$

Because of (37) and (45), if $m \in \mathcal{E}_{i} \cap \mathcal{E}_{j}$, with $i \neq j$, then

$$
\frac{2 \varepsilon}{1+L} \leq d_{f}\left(m_{i}, m_{j}\right) \leq \frac{1+L}{c_{0}}\left(d_{f}\left(m_{i}, m\right)+d_{f}\left(m, m_{j}\right)\right)<\frac{2 \varepsilon}{1+L},
$$

a contradiction. Hence the sets $\mathcal{E}_{i}$ are pairwise disjointed

$$
\mathcal{E}_{i} \cap \mathcal{E}_{j}=\emptyset \quad \text { for } i \neq j .
$$

We want to estimate from below the $\mathcal{L}^{d_{m}}$ measure of the sets $\mathcal{E}_{i}$. To this purpose observe that, from Remark 3.1.4 and for all $q_{1}, q_{2} \in \operatorname{graph}(f)$,

$$
c_{0}\left\|\mathbf{P}_{\mathbb{M}}\left(q_{1}^{-1} q_{2}\right)\right\| \leq\left\|q_{1}^{-1} q_{2}\right\| \leq\left\|q_{2}^{-1} q_{1}\right\| \leq(1+L)\left\|\mathbf{P}_{\mathbb{M}}\left(q_{2}^{-1} q_{1}\right)\right\| ;
$$

hence

and finally

$$
\left\|\mathbf{P}_{\mathbb{M}}\left(q_{1}^{-1} q_{2}\right)\right\| \leq\left((1+L) / c_{0}\right)\left\|\mathbf{P}_{\mathbb{M}}\left(q_{2}^{-1} q_{1}\right)\right\|,
$$

$$
d_{f}\left(m_{1}, m_{2}\right) \leq \frac{1+L}{c_{0}}\left\|\mathbf{P}_{\mathbb{M}}\left(q_{1}^{-1} q_{2}\right)\right\| \quad \text { for all } q_{1}, q_{2} \in \operatorname{graph}(f) .
$$

From the last inequality we have that,

$$
\mathcal{E}_{i} \supseteq\left\{m \in \mathbb{M}:\left\|\mathbf{P}_{\mathbb{M}}\left(q_{i}^{-1} q\right)\right\| \leq \frac{\varepsilon c_{0}^{2}}{(1+L)^{3}}, q=m f(m)\right\} .
$$

Observe that, for any $\delta>0$,

$$
\begin{aligned}
\left\{m \in \mathbb{M}:\left\|\mathbf{P}_{\mathbb{M}}\left(q_{i}^{-1} q\right)\right\| \leq \delta, q=m f(m)\right\} & =\mathbf{P}_{\mathbb{M}}\left(q_{i}^{-1}\{w \in \mathbb{M}:\|w\| \leq \delta\}\right) \\
& =\mathbf{P}_{\mathbb{M}}\left(q_{i}^{-1}(B(e, \delta) \cap \mathbb{M})\right) .
\end{aligned}
$$

Moreover, the map $\mathbb{M} \rightarrow \mathbb{M}$ defined as $m \mapsto \mathbf{P}_{\mathbb{M}}(q m)$ for a fixed $q \in \mathbb{G}$ has unit Jacobian. Hence, recalling also Lemma 2.2.17,

$$
\mathcal{L}^{d_{t}}\left(\mathcal{E}_{i}\right) \geq \mathcal{L}^{d_{t}}\left(B\left(e, \varepsilon c_{0}^{2}(1+L)^{-3}\right)\right)=c \varepsilon^{d_{m}} .
$$

Because $\mathcal{E}_{i} \subseteq \mathbf{P}_{\mathbb{M}}(B(e, R+1))$ there is $c=c(\mathbb{G}, \mathbb{M}, \mathbb{H}, L)$ such that

$$
N \leq c R^{d_{m}} \varepsilon^{-d_{m}}
$$

and the proof is concluded 
3.3. Examples of intrinsic Lipschitz functions. Pointwise limits of intrinsic Lipschitz functions are intrinsic Lipschitz.

Proposition 3.3.1. Let $\mathbb{M}$ and $\mathbb{H}$ be complementary subgroups of $\mathbb{G}$. Let $f, f_{n}: \mathcal{E} \subset \mathbb{M} \rightarrow \mathbb{H}$, for $n=1,2, \ldots$ and $L>0$.

(i) If the $f_{n}$ 's are intrinsic L-Lipschitz in $\mathcal{E}$ and if $f$ is the pointwise limit of $f_{n}$, then $f$ is intrinsic L-Lipschitz.

(ii) If the $f_{n}$ 's are locally equibounded and intrinsic L-Lipschitz in $\mathcal{E}$, then $\left\{f_{n}: n=1,2, \ldots\right\}$ is precompact with respect to uniform convergence in bounded subsets of $\mathcal{E}$.

Proof. The first part follows from the characterization of intrinsic Lipschitz functions given in Remark 3.1.4, together with the continuity of projection maps stated in Proposition 2.2.14.

Proposition 3.1.8 yields that the $f_{n}$ 's are locally equicontinuous, hence precompactness follows from Arzelà Ascoli Theorem.

In the following proposition we identify a large class of intrinsic Lipschitz functions. To motivate the introduction of these functions, we notice that when $\mathbb{N}$ is 1-dimensional their graph is the boundary of the 'positive part' of an intrinsic cone. With notations made precise in Section 4, we have

$$
\operatorname{graph}\left(\phi_{L}\right)=\partial C_{\mathbb{M}, \mathbb{N}}^{+}(e, 1 / L),
$$

where $C_{\mathbb{M}, \mathbb{N}}^{+}(e, 1 / L)$ is the part of the cone $C_{\mathbb{M}, \mathbb{N}}(e, 1 / L)$ contained in the 'halfspace' $S_{\mathbb{G}}^{+}$, i.e.

$$
C_{\mathbb{M}, \mathbb{N}}^{+}(e, 1 / L):=S_{\mathbb{G}}^{+} \cap C_{\mathbb{M}, \mathbb{N}}(e, 1 / L) .
$$

Differently rephrased, next Proposition states that the boundary of a 'positive' intrinsic cone is an intrinsic Lipschitz graph. This result has a technical motivation in the proof of Theorem 4.0.5.

Proposition 3.3.2. Let $\mathbb{M}$ and $\mathbb{N}$ be complementary subgroups of $\mathbb{G}$. Choose $L>0, n \in \mathbb{N}$ with $\|n\|=1$ and define $\phi_{L} \equiv \phi_{L, n}: \mathbb{M} \rightarrow \mathbb{N}$ as

$$
\phi_{L}(m):=\delta_{L\|m\|} n .
$$

If $\mathbb{N}$ is horizontal, there is $L_{1}=L_{1}(L, \mathbb{G}) \geq L$ such that $\phi_{L}$ is intrinsic $L_{1}$-Lipschitz.

The proof relies on the following two statements. The estimates in them are in the spirit of the ones in Lemma 2.2.10 but they do not follow from them. Indeed here we estimate the Euclidean norms of the vector components of $h^{-1} g h$.

Lemma 3.3.3. If $g, h \in \mathbb{G}$ then

$$
h^{-1} g h=g+\mathcal{P}(h, g),
$$

where $\mathcal{P}(h, g)=\left(\mathcal{P}^{1}(h, g), \ldots, \mathcal{P}^{\kappa}(h, g)\right)$, with $\mathcal{P}^{1}(h, g)=0$ and, for $2 \leq j \leq \kappa, \mathcal{P}^{j}(h, g)$ are (vector valued) polynomial functions homogeneous of degree $j$. Moreover, if $\mathcal{B} \subset \mathbb{G}$ is a bounded set, there exists $C_{\mathcal{B}}=C_{\mathcal{B}}(\mathbb{G})>0$ such that, for $j=2, \ldots, \kappa$,

$$
\left\|\mathcal{P}^{j}(h, g)\right\|_{n_{j}} \leq C_{\mathcal{B}}\left(\left\|g^{1}\right\|_{n_{1}}+\cdots+\left\|g^{j-1}\right\|_{n_{j-1}}\right) \quad \text { for all } h, g \in \mathcal{B} .
$$

Proof. A direct computation gives

$$
h^{-1} g h=g+\mathcal{Q}(g, h)+\mathcal{Q}(-h, g+h+\mathcal{Q}(g, h))
$$

and we set

$$
\mathcal{P}(h, g):=\mathcal{Q}(g, h)+\mathcal{Q}(-h, g+h+\mathcal{Q}(g, h)) .
$$

Hence $\mathcal{P}^{1}(h, g)=0$ because $\mathcal{Q}^{1}(h, g)=0$, for all $h, g$. To estimate $\mathcal{P}^{j}(h, g)$ for $2 \leq j \leq \kappa$ we use (5). The main point is to observe that, as a consequence of $(5), \mathcal{P}^{j}(h, g)$ is the sum of monomials each one containing a positive power of some $g_{i}$, for $1 \leq i \leq m_{j-1}$. Hence the claim follows. 
Corollary 3.3.4. There is a vector valued polynomial function $\tilde{\mathcal{P}}: \mathbb{G} \times \mathbb{G} \rightarrow \mathbb{R}^{n}$ such that

$$
h^{-1} p^{-1} q h=q-p+\tilde{\mathcal{P}}\left(h, p^{-1} q\right) \quad \text { for all } h, p, q \in \mathbb{G} .
$$

Moreover, if $\mathcal{B} \subset \mathbb{G}$ is bounded, there is $C_{\mathcal{B}}=C_{\mathcal{B}}(\mathbb{G})>0$ such that, for $j=2, \ldots, \kappa$,

$$
\left\|\tilde{\mathcal{P}}^{j}\left(h, p^{-1} q\right)\right\|_{n_{j}} \leq C_{\mathcal{B}}\left(\left\|(q-p)^{1}\right\|_{n_{1}}+\cdots+\left\|(q-p)^{j-1}\right\|_{n_{j-1}}\right) \quad \text { for all } h, p, q \in \mathcal{B} .
$$

Proof. From Lemma 3.3.3

$$
h^{-1} p^{-1} q h=p^{-1} q+\mathcal{P}\left(h, p^{-1} q\right)=q-p+\mathcal{Q}(-p, q)+\mathcal{P}\left(h, p^{-1} q\right) .
$$

Recalling (5), for all $1 \leq i \leq m_{j}$ we have homogeneous polynomials $\mathcal{R}_{\ell, n}^{i}$ such that

$$
\begin{aligned}
\mathcal{Q}_{i}(-p, q) & =\sum_{\ell, n=1}^{m_{j-1}} \mathcal{R}_{\ell, n}^{i}(-p, q)\left(-p_{\ell} q_{n}+p_{n} q_{\ell}\right) \\
& =\sum_{\ell, n=1}^{m_{j-1}} \mathcal{R}_{\ell, n}^{i}(-p, q)\left(q_{n}\left(q_{\ell}-p_{\ell}\right)-q_{\ell}\left(q_{n}+p_{n}\right)\right) .
\end{aligned}
$$

Hence

$$
\begin{aligned}
\left\|\mathcal{Q}^{j}(-p, q)\right\|_{n_{j}} & \leq c\left(\left|q_{1}-p_{1}\right|+\cdots+\left|q_{m_{j-1}}-p_{m_{j-1}}\right|\right) \\
& \leq c\left(\left\|(q-p)^{1}\right\|_{n_{1}}+\cdots+\left\|(q-p)^{j-1}\right\|_{n_{j-1}}\right) .
\end{aligned}
$$

Also $\mathcal{P}\left(h, p^{-1} q\right)$ can be estimated in the same way. Indeed, from Lemma 3.3 .3 and the preceding inequalities

$$
\begin{aligned}
\left\|\mathcal{P}^{j}\left(h, p^{-1} q\right)\right\|_{n_{j}} & \leq c_{\mathcal{B}}\left(\left\|\left(p^{-1} q\right)^{1}\right\|_{n_{1}}+\cdots+\left\|\left(p^{-1} q\right)^{j-1}\right\|_{n_{j-1}}\right) \\
& \leq c_{\mathcal{B}} \sum_{\ell=1}^{j-1}\left(\left\|(q-p)^{\ell}\right\|_{n_{\ell}}+\left\|\mathcal{Q}^{\ell}(-p, q)\right\|_{n_{\ell}}\right) \\
& \leq \tilde{c}_{\mathcal{B}} \sum_{\ell=1}^{j-1}\left(\left\|(q-p)^{\ell}\right\|_{n_{\ell}}\right) .
\end{aligned}
$$

Proof of Proposition 3.3.2. Because $\mathbb{N}$ is horizontal then $\mathbb{M}$ is a normal subgroup and, by (i) of Remark 3.1.5 we have to prove that

$$
\left\|\phi_{L}(\bar{m})^{-1} \phi_{L}(m)\right\| \leq L_{1}\left\|\phi_{L}(\bar{m})^{-1} \bar{m}^{-1} m \phi_{L}(\bar{m})\right\| \quad \text { for all } m, \bar{m} \in \mathbb{M} \text {. }
$$

We assume without loss of generality that $L=1$ and that $\|m\| \leq\|\bar{m}\|$. Finally, by homogeneity, it is enough to prove that there is $L_{1}>0$ such that

$$
\left\|\phi_{L}(\bar{m})^{-1} \phi_{L}(m)\right\| \leq L_{1}\left\|\phi_{L}(\bar{m})^{-1} \bar{m}^{-1} m \phi_{L}(\bar{m})\right\| \quad \text { for all }\|m\| \leq\|\bar{m}\|=1 .
$$

(Step I) Because $\mathbb{N}$ is horizontal it is abelian and it can be identified with a linear subspace of the first layer $\mathbb{R}^{n_{1}}$. The group operation in $\mathbb{N}$ is the usual Euclidean sum. Hence

$$
\phi_{L}(\bar{m})^{-1} \phi_{L}(m)=\phi_{L}(m)-\phi_{L}(\bar{m})=\delta_{\|m\|} n-\delta_{\|\bar{m}\|} n
$$

In the notation of $(6), n=\left(n^{1}, 0, \ldots, 0\right)$ and $\delta_{\alpha} n=\left(\alpha n^{1}, 0, \ldots, 0\right)$, for $\alpha>0$. Then

$$
\left\|\phi_{L}(\bar{m})^{-1} \phi_{L}(m)\right\|=|\|m\|-\|\bar{m}\||\|n\|=\|\bar{m}\|-\|m\|,
$$


because $\|n\|=1$. Moreover, there is $c_{1}=c_{1}(\mathbb{G})>1$, such that

$$
\|\bar{m}\|-\|m\| \leq c_{1} \sum_{j=1}^{\kappa}\left\|(\bar{m}-m)^{j}\right\|_{n_{j}}, \quad \text { for all }\|m\| \leq\|\bar{m}\|=1 .
$$

Here $\|\cdot\|$ is the group norm and $\|\cdot\|_{n}$ or $\|\cdot\|_{n_{j}}$ are the Euclidean norms in $\mathbb{R}^{n}$ or $\mathbb{R}^{n_{j}}$. Now remember that $\left|t^{p}-s^{p}\right| \leq p|t-s|$ for $0 \leq s, t \leq 1$ and $p \geq 1$. Hence if $\|m\| \leq\|\bar{m}\|=1$ and if $Q$ is the homogeneous dimension of $\mathbb{G}$,

$$
\begin{aligned}
\|\bar{m}\|-\|m\| & \leq\|\bar{m}\|^{Q}-\|m\|^{Q}=\max _{j}\left\{\varepsilon_{j}^{Q}\left\|\bar{m}^{j}\right\|_{n_{j}}^{\frac{Q}{j}}\right\}-\max _{j}\left\{\varepsilon_{j}^{Q}\left\|m^{j}\right\|_{n_{j}}^{\frac{Q}{j}}\right\} \\
& \leq \sum_{j=1}^{\kappa} \varepsilon_{j}^{Q}\left|\left\|\bar{m}^{j}\right\|_{n_{j}}^{\frac{Q}{j}}-\left\|m^{j}\right\|_{n_{j}}^{\frac{Q}{j}}\right| \\
& \leq \sum_{j=1}^{\kappa} \frac{Q \varepsilon_{j}^{j}}{j}\left|\left\|\bar{m}^{j}\right\|_{n_{j}}-\left\|m^{j}\right\|_{n_{j}}\right| \\
& \leq \sum_{j=1}^{\kappa} \frac{Q \varepsilon_{j}^{j}}{j}\left\|\bar{m}^{j}-m^{j}\right\|_{n_{j}} \\
& \leq c_{1} \sum_{j=1}^{\kappa}\left\|(\bar{m}-m)^{j}\right\|_{n_{j}},
\end{aligned}
$$

and this proves (46).

(Step II) From Corollary 3.3.4, with $h=\phi_{L}(\bar{m})$ we have

$$
\begin{aligned}
\phi_{L}(\bar{m})^{-1} \bar{m}^{-1} m \phi_{L}(\bar{m}) & = \\
& \left((\bar{m}-m)^{1},(\bar{m}-m)^{2}+\tilde{\mathcal{P}}^{2}\left(\phi_{L}(\bar{m}), \bar{m}^{-1} m\right), \ldots,(\bar{m}-m)^{\kappa}+\tilde{\mathcal{P}}^{\kappa}\left(\phi_{L}(\bar{m}), \bar{m}^{-1} m\right)\right) .
\end{aligned}
$$

Hence, there is $c_{2}=c_{2}(\mathbb{G})>0$ such that

$$
\begin{gathered}
\left\|\phi_{L}(\bar{m})^{-1} \bar{m}^{-1} m \phi_{L}(\bar{m})\right\| \\
\geq c_{2}\left(\left\|(\bar{m}-m)^{1}\right\|_{n_{1}}+\left\|(\bar{m}-m)^{2}+\tilde{\mathcal{P}}^{2}\left(\phi_{L}(\bar{m}), \bar{m}^{-1} m\right)\right\|_{n_{2}}+\ldots\right. \\
\left.\cdots+\left\|(\bar{m}-m)^{k}+\tilde{\mathcal{P}}^{\kappa}\left(\phi_{L}(\bar{m}), \bar{m}^{-1} m\right)\right\|_{n_{\kappa}}\right):=I
\end{gathered}
$$

because all the terms on the right hand side are bounded.

We want to prove that $\sum_{j=1}^{\kappa}\left\|(\bar{m}-m)^{j}\right\|_{n_{j}}$ is bounded from above by a constant times the right hand side $I$ of $(47)$. Once more from Corollary 3.3.4, there is $c_{3}=c_{3}(\mathbb{G})>1$ such that

$$
\left\|\tilde{\mathcal{P}}^{j}\left(\phi_{L}(\bar{m}), \bar{m}^{-1} m\right)\right\|_{n_{j}} \leq c_{3}\left(\left\|(m-\bar{m})^{1}\right\|_{n_{1}}+\cdots+\left\|(m-\bar{m})^{j-1}\right\|_{n_{j-1}}\right)
$$

for $j=2, \ldots, \kappa$. Here $c_{3}$ is the constant $C_{\mathcal{B}}$ of Corollary 3.3.4 where $\mathcal{B}$ is the unit ball so containing $\phi_{L}(\bar{m}), m$ and $\bar{m}$. Then we prove

$$
\begin{aligned}
& \left\|(\bar{m}-m)^{1}\right\|_{n_{1}} \leq I, \\
& \left\|(\bar{m}-m)^{j}\right\|_{n_{j}} \leq 2 c_{3}\left(1+2 c_{3}\right)^{j-2} I \quad \text { for } 2 \leq j \leq \kappa .
\end{aligned}
$$

The first line is clear. To obtain also the second one we argument by induction on $j$. Fix $\ell$ with $2 \leq \ell \leq \kappa$ and assume that (49) is true for $1 \leq j<\ell$. Observe that if

$$
\left\|(\bar{m}-m)^{\ell}+\tilde{\mathcal{P}}^{\ell}\left(\phi_{L}(\bar{m}), \bar{m}^{-1} m\right)\right\|_{n_{\ell}} \geq \frac{1}{2}\left\|(\bar{m}-m)^{\ell}\right\|_{n_{\ell}}
$$


then (49) follows with $j=\ell$. On the contrary, if

$$
\left\|(\bar{m}-m)^{\ell}+\tilde{\mathcal{P}}^{\ell}\left(\phi_{L}(\bar{m}), \bar{m}^{-1} m\right)\right\|_{n_{\ell}}<\frac{1}{2}\left\|(\bar{m}-m)^{\ell}\right\|_{n_{\ell}}
$$

then

$$
\left\|\tilde{\mathcal{P}}^{\ell}\left(\phi_{L}(\bar{m}), \bar{m}^{-1} m\right)\right\|_{n_{\ell}}>\frac{1}{2}\left\|(\bar{m}-m)^{\ell}\right\|_{n_{\ell}}
$$

hence, by (48) and by the induction assumption,

$$
\begin{aligned}
\left\|(\bar{m}-m)^{\ell}\right\|_{n_{\ell}} & \leq 2 c_{3}\left(\left\|(\bar{m}-m)^{1}\right\|_{n_{1}}+\cdots+\left\|(\bar{m}-m)^{\ell-1}\right\|_{n_{\ell-1}}\right) \leq c I \\
& \leq 2 c_{3}\left(1+2 c_{3}+\ldots 2 c_{3}\left(1+2 c_{3}\right)^{\ell-3}\right) I \\
& =2 c_{3}\left(1+2 c_{3}\right)^{\ell-2} I .
\end{aligned}
$$

Eventually from (49) we get

$$
\sum_{j=1}^{\kappa}\left\|(\bar{m}-m)^{j}\right\|_{n_{j}} \leq c_{4} I
$$

where $c_{4}=\left(1+2 c_{3}\right)^{\kappa}$. Now the Proposition is proved with $L_{1}=\frac{c_{1} c_{4}}{c_{2}}$.

\section{1-Codimensional Intrinsic Graphs and Extension of Lipschitz Functions}

Through all this section $\mathbb{G}=\mathbb{M} \cdot \mathbb{N}$, where as usual $\mathbb{M}$ and $\mathbb{N}$ are complementary homogeneous subgroups, but here we assume also that $\mathbb{N}$ is one dimensional and (consequently) horizontal. Precisely we assume the existence of $V \in \mathfrak{g}_{1}$ such that $\mathbb{N}=\{\exp (t V), t \in \mathbb{R}\}$. Under these assumptions, $\mathbb{M}$ is always a normal subgroup since, as observed in Remark 2.2.1, it contains the whole strata $\mathbb{G}^{2}, \ldots, \mathbb{G}^{\kappa}$. As pointed out in the Introduction, graphs corresponding to such a decomposition, can be viewed as graphs of "real valued functions". The core of this Section is therefore the proof of an extension theorem for "real valued intrinsic Lipschitz functions", that is the counterpart of the classical McShane extension theorem in metric spaces.

A couple of remarks are now in order: first of all, while any extension theorem for real valued metric Lipschitz functions trivially yields an analogous theorem for (finite dimensional) vectorvalued Lipschitz functions, when dealing with intrinsic Lipschitz functions the situation is utterly different. Indeed, the property of being intrinsic Lipschitz cannot be read in terms of "having intrinsic Lipschitz components". The second remark is that the extension property fails to hold for intrinsic Lipschitz functions acting between subgroups associated with arbitrary decompositions. Indeed, the extension property fails already for metric Lipschitz functions acting from $\mathbb{R}^{n}$ to $\mathbb{H}^{m}$, with $n>m([6])$. Therefore, if we take $\mathbb{G}=\mathbb{R}^{n} \times \mathbb{H}^{m}$, we obtain easily a counter-example.

Since $\mathbb{N}=\{\exp t V\}, \mathbb{N}$ can be identified with $\mathbb{R}$ so that it carries an order and we can define the supremum and the infimum of families of $\mathbb{N}$-valued functions. If $f_{\beta}: \mathbb{M} \rightarrow \mathbb{N}$ for $\beta \in B$ with $f_{\beta}(m)=\exp \left(\varphi_{\beta}(m) V\right)$ and $\varphi_{\beta}: \mathbb{M} \rightarrow \mathbb{R}$, we define $\inf _{\beta \in B} f_{\beta}: \mathbb{M} \rightarrow \mathbb{N}$ as

$$
\inf _{\beta \in B} f_{\beta}(m):=\exp \left(\inf _{\beta \in B} \varphi_{\beta}(m) V\right)
$$

for all $m \in \mathbb{M}$ s.t. $\inf _{\beta \in B} \varphi_{\beta}(m)$ is finite. Analogously are defined $\sup _{\beta \in B} f_{\beta}, \max \left\{f_{\beta_{1}}, f_{\beta_{2}}\right\}$, $\min \left\{f_{\beta_{1}}, f_{\beta_{2}}\right\}$, etc.

Theorem 4.0.5. Let $\mathbb{M}$ and $\mathbb{N}$ be complementary subgroups with $\mathbb{N}$ one dimensional. Let $\mathcal{B} \subset \mathbb{M}$ be a Borel subset of $\mathbb{M}$ and $f: \mathcal{B} \rightarrow \mathbb{N}$ be an intrinsic L-Lipschitz function. Then there are $\tilde{f}: \mathbb{M} \rightarrow \mathbb{N}$ 
and $\tilde{L}=\tilde{L}(L, \mathbb{G}, \mathbb{M}, \mathbb{N}) \geq L$ such that

$$
\begin{aligned}
& \tilde{f} \text { is intrinsic } \tilde{L} \text {-Lipschitz in } \mathbb{M} \text {, } \\
& \tilde{f}(m)=f(m) \quad \text { for all } m \in \mathcal{B} .
\end{aligned}
$$

We need a few lemmas and notations in order to prove Theorem 4.0.5. Denote by $S_{\mathbb{G}}^{+}(\mathbb{M}, \mathbb{N})$ the 'halfspace'

$$
S_{\mathbb{G}}^{+}(\mathbb{M}, \mathbb{N})=\left\{g: g_{\mathbb{N}}=\exp (t V), \text { with } t \geq 0\right\} .
$$

$S_{\mathbb{G}}^{-}(\mathbb{M}, \mathbb{N})$ is the analogous one with $t \leq 0$. If $C_{\mathbb{M}, \mathbb{N}}(p, \alpha)$ is an intrinsic cone with one dimensional axis $\mathbb{N}$, we denote

$$
C_{\mathbb{M}, \mathbb{N}}^{ \pm}(e, \alpha):=C_{\mathbb{M}, \mathbb{N}}(e, \alpha) \cap S_{\mathbb{G}}^{ \pm}(\mathbb{M}, \mathbb{N})
$$

and $C_{\mathbb{M}, \mathbb{N}}^{ \pm}(p, \alpha):=p \cdot C_{\mathbb{M}, \mathbb{N}}^{ \pm}(e, \alpha)$. Notice also that

$$
\partial C_{\mathbb{M}, \mathbb{N}}^{+}(e, 1 / L)=\operatorname{graph}\left(\phi_{L}\right),
$$

where the functions $\phi_{L}: \mathbb{M} \rightarrow \mathbb{N}$, defined as $\phi_{L}(m)=\delta_{L\|m\|}(\exp V)=\exp (L\|m\| V)$, are the functions considered in Proposition 3.3.2.

Lemma 4.0.6. For each $\alpha>0$ there is $\alpha_{1}=\alpha_{1}(\alpha, \mathbb{G}, \mathbb{M}, \mathbb{N}), 0<\alpha_{1} \leq \alpha$, such that

$$
\begin{array}{ll}
C_{\mathbb{M}, \mathbb{N}}^{+}\left(g n, \alpha_{1}\right) \subset C_{\mathbb{M}, \mathbb{N}}^{+}(g, \alpha), \quad & \text { for all } g \in \mathbb{G} \text { and } n=\exp (t V) \in \mathbb{N}, \text { with } t>0, \\
C_{\mathbb{M}, \mathbb{N}}^{-}\left(g n, \alpha_{1}\right) \subset C_{\mathbb{M}, \mathbb{N}}^{-}(g, \alpha), & \text { for all } g \in \mathbb{G} \text { and } n=\exp (t V) \in \mathbb{N}, \text { with } t<0 .
\end{array}
$$

Proof. By left translation invariance, it is enough to prove that

$$
C_{\mathbb{M}, \mathbb{N}}^{+}\left(n, \alpha_{1}\right) \subset C_{\mathbb{M}, \mathbb{N}}^{+}(e, \alpha), \quad \text { for all } n=\exp (t V) \in \mathbb{N}, \text { with } t>0 .
$$

Let $p=p_{\mathbb{M}} p_{\mathbb{N}} \in C_{\mathbb{M}, \mathbb{N}}^{+}\left(e, \alpha_{1}\right)$, we have to prove that $n p \in C_{\mathbb{M}, \mathbb{N}}^{+}(e, \alpha)$. Because $\mathbb{M}$ is a normal subgroup, $(n p)_{\mathbb{M}}=n p_{\mathbb{M}} n^{-1}$ and $(n p)_{\mathbb{N}}=n p_{\mathbb{N}}$.

Because $\operatorname{dim} \mathbb{N}=1$ and $\mathbb{N}$ is horizontal,

$$
\left\|(n p)_{\mathbb{N}}\right\|=\left\|p_{\mathbb{N}}\right\|+\|n\| .
$$

Indeed, if $n=\exp \theta V$ and $\bar{n}=\exp \bar{\theta} V$, then $n \bar{n}=\exp ((\theta+\bar{\theta}) V)$ and $\|n \bar{n}\|=|\theta+\bar{\theta}|=|\theta|+|\bar{\theta}|$ when $\theta$ and $\bar{\theta}$ are positive.

By Lemma 2.2.10 there is $C=C(\mathbb{G})>0$ such that

$$
\left\|(n p)_{\mathbb{M}}\right\|=\left\|n p_{\mathbb{M}} n^{-1}\right\| \leq\left\|p_{\mathbb{M}}\right\|+C\left(\|n\|^{1 / \kappa}\left\|p_{\mathbb{M}}\right\|^{(\kappa-1) / \kappa}+\|n\|^{(\kappa-1) / \kappa}\left\|p_{\mathbb{M}}\right\|^{1 / \kappa}\right) .
$$

For $\kappa \geq 2$ there is $c=c(\kappa) \geq 1$ such that $s^{1 / \kappa} t^{(\kappa-1) / \kappa}+t^{1 / \kappa} s^{(\kappa-1) / \kappa} \leq \varepsilon t+c \varepsilon^{1-\kappa} s$, for all $s, t \geq 0$ and for all $\varepsilon>0$. Hence we have

$$
\left\|(n p)_{\mathbb{M}}\right\| \leq\left(1+C c \varepsilon^{1-\kappa}\right)\left\|p_{\mathbb{M}}\right\|+C \varepsilon\|n\| \leq \alpha_{1}\left(1+C c \varepsilon^{1-\kappa}\right)\left\|p_{\mathbb{N}}\right\|+C \varepsilon\|n\|,
$$

because $p \in C_{\mathbb{M}, \mathbb{N}}^{+}\left(e, \alpha_{1}\right)$. Now choose $\varepsilon=\alpha / C$ and $\alpha_{1}=\alpha /\left(1+C c \varepsilon^{1-\kappa}\right)$ to get

$$
\left\|(n p)_{\mathbb{M}}\right\| \leq \alpha\left\|p_{\mathbb{N}}\right\|+\alpha\|n\|=\alpha\left\|(n p)_{\mathbb{N}}\right\|
$$

that shows that $n p \in C_{\mathbb{M}, \mathbb{N}}^{+}(e, \alpha)$ and completes the proof for the "positive" cones. The case of the "negative" cones is completely analogous.

We can characterize $\mathbb{N}$ valued intrinsic Lipschitz functions using the fact that subgraphs and supergraphs contain half cones. Precisely, for $f: \mathcal{U} \subset \mathbb{M} \rightarrow \mathbb{N}$, with $f(m)=\exp (\varphi(m) V)$ and $\varphi: \mathcal{U} \rightarrow \mathbb{R}$, we define the supergraph $E_{f}^{+}$and the subgraph $E_{f}^{-}$of $f$ as

$$
E_{f}^{-}:=\{m \exp (t V): m \in \mathcal{U}, t<\varphi(m)\}, \quad E_{f}^{+}:=\{m \exp (t V): m \in \mathcal{U}, t>\varphi(m)\} .
$$


Notice that, if $f: \mathbb{M} \rightarrow \mathbb{N}$ is continuous,

$$
\overline{E_{f}^{-}}=\{m \exp (t V): m \in \mathbb{M}, t \leq \varphi(m)\}, \quad \overline{E_{f}^{+}}=\{m \exp (t V): m \in \mathbb{M}, t \geq \varphi(m)\} .
$$

Lemma 4.0.7. $f: \mathcal{U} \subseteq \mathbb{M} \rightarrow \mathbb{N}$ is intrinsic L-Lipschitz in $\mathcal{U}$ if and only if for all $m \in \mathbb{M}$

$$
C_{\mathbb{M}, \mathbb{N}}^{+}(m f(m), 1 / L) \subset \overline{E_{f}^{+}} \quad \text { and } \quad C_{\mathbb{M}, \mathbb{N}}^{-}(m f(m), 1 / L) \subset \overline{E_{f}^{-}} .
$$

Proof. Let $f(m):=\exp (\varphi(m) V)$, where $\varphi: \mathbb{M} \rightarrow \mathbb{R}$. If $f$ is intrinsic $L$-Lipschitz then for all $\alpha<1 / L$ and for all $m \in \mathbb{M}$

$$
C_{\mathbb{M}, \mathbb{N}}(m f(m), \alpha) \cap \operatorname{graph}(f)=\{m f(m)\} .
$$

Assume by contradiction that there is $\bar{m} \in \mathbb{M}$ and $\bar{t}>\varphi(\bar{m}) \in \mathbb{R}$ such that

$$
\bar{m} \cdot \exp (\bar{t} V) \in C_{\mathbb{M}, \mathbb{N}}^{+}(\bar{m} f(\bar{m}), \alpha) \cap E_{f}^{-} ;
$$

but then $\bar{m} \cdot \exp (t V) \in C_{\mathbb{M}, \mathbb{N}}^{+}(\bar{m} f(\bar{m}), \alpha)$, for all $t \geq \bar{t}$, in particular

$$
\bar{m} \cdot \exp (\varphi(\bar{m}) V) \in C_{\mathbb{M}, \mathbb{N}}^{+}(\bar{m} f(\bar{m}), \alpha)
$$

contraddicting (52).

On the contrary, if (51) is true, then for all $0<\alpha<L$,

$$
\begin{aligned}
C_{\mathbb{M}, \mathbb{N}}(\bar{m} f(\bar{m}), \alpha) & =C_{\mathbb{M}, \mathbb{N}}^{-}(\bar{m} f(\bar{m}), \alpha) \cup C_{\mathbb{M}, \mathbb{N}}^{+}(\bar{m} f(\bar{m}), \alpha) \\
& \subset E_{f}^{-} \cup E_{f}^{+} \cup\{m f(m)\}
\end{aligned}
$$

proving that $f$ is intrinsic $L$-Lipschitz.

Proposition 4.0.8. Assume that $f_{\beta}: \mathbb{M} \rightarrow \mathbb{N}$ for $\beta \in B$ is a family of intrinsic L-Lipschitz functions. For all $L>0$ there is $\tilde{L}=\tilde{L}(L, \mathbb{G}, \mathbb{M}, \mathbb{N}) \geq L$ such that, if

$$
f:=\inf _{\beta \in B} f_{\beta},
$$

then either $f \equiv-\infty$ or $f$ is defined on all of $\mathbb{M}$ and it is intrinsic $\tilde{L}$-Lipschitz.

Proof. Clearly,

$$
E_{f}^{-}=\bigcap_{\beta} E_{f_{\beta}}^{-}, \quad E_{f}^{+}=\bigcup_{\beta} E_{f_{\mathfrak{g}}}^{+} .
$$

Assume there is $\bar{m} \in \mathbb{M}$ such that $f(\bar{m}) \in \mathbb{N}$ (i.e. the infimum is not $-\infty$ in at least one point). Then, from Lemmas 4.0.6 and 4.0.7, for all $\alpha<1 / L$

$$
C_{\bar{M}, \mathbb{N}}^{-}\left(\bar{m} f(\bar{m}), \alpha_{1}\right) \subset C_{\mathbb{M}, \mathbb{N}}^{-}\left(\bar{m} f_{\beta}(\bar{m}), \alpha\right) \subset E_{f_{\beta}}^{-} .
$$

Hence

$$
C_{\mathbb{M}, \mathbb{N}}^{-}\left(\bar{m} f(\bar{m}), \alpha_{1}\right) \subset \bigcap_{\beta} E_{f_{\beta}}^{-}=E_{f}^{-} .
$$

It follows, in particular, that $f(m) \in \mathbb{N}$ for all $m \in \mathbb{M}$ and consequently we can repeat the preceding argument for all $m \in \mathbb{M}$ obtaining

$$
C_{\mathbb{M}, \mathbb{N}}^{-}\left(m f(m), \alpha_{1}\right) \subset E_{f}^{-} \quad \text { for all } m \in \mathbb{M} .
$$

On the other side

$$
C_{\mathbb{M}, \mathbb{N}}^{+}(m f(m), \alpha) \subset E_{f_{\beta}}^{+} \quad \text { for all } m \in \mathbb{M} \text { and } \beta \in B,
$$

so that

$$
C_{\mathbb{M}, \mathbb{N}}^{+}(m f(m), \alpha) \subset E_{f}^{+} \quad \text { for all } m \in \mathbb{M} .
$$

The claim then follows from Lemma 4.0.7. 
Proof of Theorem 4.0.5. For each $\bar{m} \in \mathcal{B}$ let $\phi_{L, \bar{m}}: \mathbb{M} \rightarrow \mathbb{N}$ be the translated function

$$
\phi_{L, \bar{m}}(m):=f(\bar{m}) \phi_{L}\left(f(\bar{m})^{-1} \bar{m}^{-1} m f(\bar{m})\right),
$$

where $\phi_{L}$ was introduced in Proposition 3.3.2. By Proposition 2.2.18 and Remark 2.2.20,

$$
\operatorname{graph}\left(\phi_{L, \bar{m}}\right)=\bar{m} f(\bar{m}) \operatorname{graph}\left(\phi_{L}\right) \text {. }
$$

Hence, from (50),

$$
\operatorname{graph}\left(\phi_{L, \bar{m}}\right)=\partial C_{\mathbb{M}, \mathbb{N}}^{+}(\bar{m} f(\bar{m}), 1 / L) .
$$

Moreover, from Proposition 3.3.2 we know that $\phi_{L}$ is intrinsic $L_{1}$-Lipschitz, hence all the translated functions $\phi_{L, \bar{m}}$ are intrinsic $L_{1}$-Lipschitz.

Define $\theta_{\bar{m}}: \mathbb{M} \rightarrow \mathbb{R}$ and $\varphi: \mathcal{B} \rightarrow \mathbb{R}$ such that

$$
\phi_{L, \bar{m}}(m)=\exp \left(\theta_{\bar{m}}(m) V\right) \text { and } f(m)=\exp (\varphi(m) V) .
$$

Since $\phi_{L, \bar{m}}(\bar{m})=f(\bar{m})$, we have

$$
\theta_{\bar{m}}(\bar{m})=\varphi(\bar{m}), \quad \text { for all } \bar{m} \in \mathcal{B} .
$$

We define $\tilde{f}: \mathbb{M} \rightarrow \mathbb{N}$ as

$$
\tilde{f}(m):=\inf _{\bar{m} \in \mathcal{B}} \phi_{L, \bar{m}}(m), \quad \text { for all } m \in \mathbb{M} .
$$

We want to show now that $\tilde{f}(m)=f(m)$ for all $m \in \mathcal{B}$. Given (54), it is enough to show

$$
\theta_{\bar{m}}(m) \geq \varphi(m), \quad \text { for all } m, \bar{m} \in \mathcal{B} \text {. }
$$

Now, because $f$ is intrinsic $L$-Lipschitz, keeping in mind (54),

$$
\left|\varphi(m)-\theta_{\bar{m}}(\bar{m})\right|=|\varphi(m)-\varphi(\bar{m})|=\left\|f(\bar{m})^{-1} f(m)\right\| \leq L\left\|f(\bar{m})^{-1} \bar{m}^{-1} m f(\bar{m})\right\| .
$$

On the other hand, from (53), $\theta_{\bar{m}}(m) \geq \theta_{\bar{m}}(\bar{m})$ and

$$
\begin{aligned}
\theta_{\bar{m}}(m)-\theta_{\bar{m}}(\bar{m}) & =\left\|\phi_{L, \bar{m}}(\bar{m})^{-1} \phi_{L, \bar{m}}(m)\right\|=L\left\|\phi_{L, \bar{m}}(\bar{m})^{-1} \bar{m}^{-1} m \phi_{L, \bar{m}}(\bar{m})\right\| \\
& =L\left\|f(\bar{m})^{-1} \bar{m}^{-1} m f(\bar{m})\right\| .
\end{aligned}
$$

Combining (56) and (57) we get (55).

Finally we apply Proposition 4.0 .8 with $L=L_{1}$ to get that $\tilde{f}$ is intrinsic $\tilde{L}$-Lipschitz for $\tilde{L}:=$ $\tilde{L}\left(L_{1}, \mathbb{G}, \mathbb{M}, \mathbb{N}\right)$.

\section{REFERENCES}

[1] R.Abraham, J.E.Marsden, T.Raţiu, Manifolds, tensor analysis, and applications. Global Analysis Pure and Applied: Series B, 2. Addison-Wesley Publishing Co., Reading, Mass. (1983).

[2] L.Ambrosio, B.Kirchheim, Rectifiable sets in metric and Banach spaces, Math. Annalen, 318 (2000), $527-555$.

[3] L.Ambrosio, B.Kirchheim, Currents in metric spaces, Acta Math. 185 (2000), 1-80.

[4] G.ArenA, Intrinsic Graphs, Convexity and Calculus on Carnot Groups, PhD Thesis, University of Trento, (2008).

[5] G.Arena, R.Serapioni, Intrinsic regular submanifolds in Heisenberg groups are differentiable graphs, Calc. Var. Partial Differential Equations 35 (2009), no. 4, 517-536.

[6] Z.BAlogh, K. FÄssler, Rectifiability and Lipschitz extensions into the Heisenberg group, Math. Z. 263 (2009), no. 3, 673-683.

[7] Z.Balogh, K. Fässler, P. Mattila, J.Tyson, Projection and slicing theorems in Heisenberg groups, Adv. Math. 231 (2012), no. 2, 569-604.

[8] F.Bigolin, D.Vittone, Some remarks about parametrizations of intrinsic regular surfaces in the Heisenberg group, Publ. Mat. 54 (2010), no. 1, 159-172.

[9] A.Bonfiglioli, E.Lanconelli, F.Uguzzoni, Stratified Lie Groups and Potential Theory for their SubLaplacians, Springer Monographs in Mathematics, Springer-Verlag Berlin Heidelberg, New York (2007).

[10] A.Baldi, B.Franchi, N.TChou, M.C.Tesi, Compensated compactness for differential forms in Carnot groups and applications, Adv. Math., 223 (5) (2010), 1555-1607. 
[11] G. Citti, A. Sarti, A cortical based model of perceptual completion in the roto-translation space, J. Math. Imaging Vision, Journal of Mathematical Imaging and Vision, 24, (2006), 3, 307-326.

[12] J.Cheeger, B. Kleiner,Differentiating maps into $L^{1}$, and the geometry of BV functions, Ann. of Math. (2) 171 (2010), no. 2, 1347-1385.

[13] G.Citti, M.Manfredini, Implicit function theorem in Carnot-Carathodory spaces. Commun. Contemp. Math. 8 (2006), no. 5, 657-680.

[14] G.Citti, M.Manfredini, Blow-up in non homogeneous Lie groups and rectifiability. Houston J. Math. 31 (2005), no. $2,333-353$.

[15] D.Cole, S.D.PAuls, $C^{1}$ hypersurfaces of the Heisenberg group are N-rectifiable, Houston J.Math. 32 (2006), no. 3, 307-326.

[16] G. Csató, B. Dacorogna, O. Kneuss, The pullback equation for differential forms. Progress in Nonlinear Differential Equations and their Applications, 83. Birkhuser/Springer, New York (2012).

[17] H.Federer, Geometric Measure Theory, Springer, (1969).

[18] G.B.Folland, Subelliptic estimates and function spaces on nilpotent Lie groups, Ark. Mat., 13 (1975), $161-207$.

[19] B.Franchi, M.Marchi, R.Serapioni, Differentiability and Approximate Differentiability for intrinsic Lipschitz functions in Carnot groups and a Rademacher Theorem, Anal. Geom. Metr. Spaces, 2 (2014), 258-281.

[20] B. Franchi, V. Penso, R. Serapioni, Remarks on Lipschitz domains in Carnot groups, in Geometric control and sub-Riemannian geometry (U. Boscain, J.-P. Gauthier, A. Sarychev, M. Sigalotti, G. Stefani, eds.), Springer INdAM Series, Springer, 2013, pp. 153-166.

[21] B.Franchi, R.Serapioni, F.Serra Cassano, Rectifiability and perimeter in the Heisenberg group, Math. Ann. 321 (2001), 479-531.

[22] B.Franchi, R.Serapioni, F.Serra Cassano, Regular hypersurfaces, Intrinsic Perimeter and Implicit Function Theorem in Carnot Groups, Comm. Anal. Geom., 11 (2003), no 5, 909-944.

[23] B.Franchi, R.Serapioni, F.Serra Cassano, On the Structure of Finite Perimeter Sets in Step 2 Carnot Groups, The Journal of Geometric Analysis. 13 (2003), no. 3, 421-466.

[24] B.Franchi, R.Serapioni, F.Serra Cassano, Intrinsic Lipschitz graphs in Heisenberg groups. J. Nonlinear Convex Anal. 7 (2006), no. 3, 423-441.

[25] B.Franchi, R.Serapioni, F.Serra Cassano, Regular submanifolds, graphs and area formula in Heisenberg Groups. Advances in Math. 211 (2007), no.1, 152-203.

[26] B.Franchi, R.Serapioni, F.Serra Cassano, Rademacher theorem for intrinsic Lipschitz continuous functions, J. Geom. Anal. 21 (2011), 1044-1084.

[27] B.Franchi, M.C.Tesi, Wave and Maxwell's Equations in Carnot Groups, Commun. Contemp. Math., 14 (5) (2012).

[28] M.Gromov, Metric structures for Riemannian and Non Riemannian Spaces, Progress in Mathematics, 152, Birkhauser Verlag, Boston, (1999).

[29] S. Helgason, Differential Geometry, Lie Groups, and Symmetric Spaces, Academic Press, New York (1978).

[30] I.N.Herstein, Topics in algebra, Blaisdell Publishing Company, London (1964).

[31] B.Kirchheim, F.SerRa CASSAno, Rectifiability and parameterizations of intrinsically regular surfaces in the Heisenberg group, Ann. Scuola Norm. Sup. Pisa, Cl.Sc. (5) III, (2005), 871-896.

[32] V.Magnani, Elements of Geometric Measure Theory on Sub-Riemannian Groups, Tesi di Perfezionamento, Scuola Normale Superiore, Pisa, (2003).

[33] V.Magnani, Towards differential calculus in stratified groups, Journal of Australian Mathematical Society, 95 no.1, (2013), 76-128.

[34] J.Mitchell, On Carnot-Carathèodory metrics, J.Differ. Geom. 21 (1985), 35-45.

[35] R.Montgomery, A Tour of Subriemannian Geometries, Their Geodesics and Applications, Mathematical Surveys and Monographs 91, American Mathematical Society, Providence, RI (2002).

[36] P.PAnsu, Métriques de Carnot-Carathéodory et quasiisométries des espaces symétriques de rang un, Ann. of Math. 129 (1989), 1-60.

[37] S.D.PAuls, A notion of rectifiability modelled on Carnot groups, Indiana Univ. Math. J. 53 (2004), no. 1, 49-81.

[38] M.Rumin, Around heat decay on forms and relations of nilpotent Lie groups, In Séminaire de Théorie Spectrale et Géométrie, 19, Univ. Grenoble I, (2001), 123-164.

[39] M.Rumin, Differential geometry on C-C spaces and application to the Novikov-Shubin numbers of nilpotent Lie groups, C. R. Acad. Sci. Paris Ser. I Math., 329 (1999), 985-990.

[40] S. SEMmes, On the nonexistence of bi-Lipschitz parameterizations and geometric problems about A-weights Rev. Mat. Iberoamericana 12 (1996), no. 2, 337-410.

[41] E.M.Stein, Harmonic Analysis: Real variable methods, orthogonality and oscillatory integrals, Princeton University Press, Princeton (1993).

[42] N.Th.Varopoulos, L.Saloff-Coste, T.Coulhon, Analysis and Geometry on Groups, Cambridge University Press, Cambridge, (1992) 
Bruno Franchi: Dipartimento di Matematica, Università di Bologna, Piazza di porta San Donato, 5 , 40126 BolognA, ItALY

E-mail address: bruno.franchi@unibo.it

Raul Paolo Serapioni: Dipartimento di Matematica, Università di Trento, Povo, Via Sommarive 14, 38123, Trento, Italy

E-mail address: serapion@science.unitn.it 